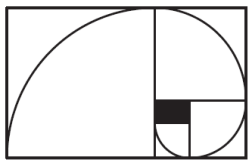

\title{
Introduction to (generalized) Gibbs measures
}

\section{Arnaud Le Ny}

\begin{abstract}
In this monograph, we survey some key issues of the theory of Gibbsian and non-Gibbsian measures in finite-spin lattice systems. While non-Gibbsian measures are truly only the object of the last chapter, the material of the first chapters is selected with generalized Gibbs measures in mind. The topics of Gibbsian theory are then chosen for their foundational or contrasting role with respect to the measures analyzed in the final chapter, including in particular more detailed parts as e.g. the proof of the Choquet decomposition of Gibbs measures in Chapter 2, a proof of the Kozlov theorem in Chapter 3, under a slightly novel presentation that serves to introduce a telescoping procedure needed for generalized Gibbs measures in Chapter 5, and a careful discussion of the variational principle in Chapter 4. This monograph covers also the contents of mini-courses given in 2007 at the universities UFMG (Belo Horizonte) and UFRGS (Porto Alegre), whose aim was to convey, in a relatively short number of lectures, the heart of the theory needed to understand Gibbsianness and non-Gibbsianness.
\end{abstract}





\section{Contents}

1 Introduction 5

2 Topology and measures on product spaces 9

2.1 Configuration space: set-up and notations . . . . . . . . . . 9

2.1.1 Lattices . . . . . . . . . . . . . . . . . . . . . . 9

2.1 .2 Single-spin state-space . . . . . . . . . . . . . . 10

2.1.3 Configuration space . . . . . . . . . . 10

2.2 Measurable properties of the configuration space . . . . . . 10

2.3 Topological properties of the configuration space . . . . . 13

2.3.1 Product topology . . . . . . . . . . . . . . 13

2.3.2 Quasilocality for functions . . . . . . . . . . . 14

2.3.3 Weak convergence of probability measures . . . . . . 15

2.4 Probability theory on infinite product spaces . . . . . . . 17

2.4.1 Kolmogorov's consistency . . . . . . . . . . . . 17

2.4.2 Regular versions of conditional probabilities . . . . . 18

2.4.3 DLR-consistency and specifications . . . . . . . . . 21

2.4 Examples ..................... 23

2.5 Convexity theory of DLR-measures . . . . . . . . . . . . 28

2.5.1 Choquet simplex of DLR-measures . . . . . . . . 29

2.5.2 Selections by boundary conditions . . . . . . . . . 40

2.5.3 Ergodic vs. extremal DLR measures . . . . . . . . 41

3 Quasilocal and Gibbs measures $\quad 44$

3.1 Quasilocality for measures and specifications . . . . . . . . 44

3.1.1 Essential continuity of conditional probabilities . . . 44

3.1.2 Existence results in the quasilocal framework . . . . 46

3.2 Infinite-volume Gibbs measures . . . . . . . . . . . . . . . . 46

3.2.1 Equilibrium states at finite volume . . . . . . . . . 46

3.2.2 Gibbs specifications and infinite-volume Gibbs measures . . . . . . . . . . . . . . . . . 48

3.2.3 Gibbs representation theorem . . . . . . . . . 60 
4 Equilibrium approach $\quad \mathbf{7 0}$

4.1 Thermodynamic properties . . . . . . . . . . . 71

4.1.1 Thermodynamic functions . . . . . . . . . . . . 71

4.1.2 Variational principles . . . . . . . . . . . 73

4.2 Topological criterion for variational principles . . . . . . . . 74

4.2.1 Second part of the variational principle: General cri-

terion ................. 75

4.2.2 First part of the variational principle: General criterion ................. 77

4.2.3 Application: Variational principle for translationinvariant quasilocal measures . . . . . . . . 81

4.3 More on equilibrium: LDP and Stochastic Ising models . . 86

4.3.1 Large deviation properties . . . . . . . . . . . 86

4.3.2 Stochastic Ising models . . . . . . . . . . . . 87

5 Generalized Gibbs measures $\quad 90$

5.1 Heuristics . . . . . . . . . . . . . . . . . . 90

5.2 RG pathologies and non-Gibbsianness . . . . . . . . . . 93

5.2.1 Decimation of the $2 d$ ferromagnetic Ising model . . . 93

5.2.2 General RG transformations, examples and results . 104

5.2.3 Stochastic evolutions of Gibbs measures . . . . . . . 109

5.2.4 Joint measure of short range disordered systems . . 111

5.2.5 Other sources of non-Gibbsianness . . . . . . . . . . 112

5.3 Generalized Gibbs measures . . . . . . . . . . . . . . . . . . 112

5.3.1 Dobrushin program of restoration, Part I . . . . . . 113

5.3.2 Dobrushin program of restoration, part II . . . . . . 114 


\section{Chapter 1}

\section{Introduction}

These notes have been written to complete the mini-course "Introduction to (generalized) Gibbs measures" given at the universities UFMG (Universidade Federal de Minas Gerais, Belo Horizonte) and UFRGS (Universidade Federal do Rio Grande do Sul, Porto Alegre) during the first semester 2007. The main goal of the lectures was to describe Gibbs and generalized Gibbs measures on lattices at a rigorous mathematical level, as equilibrium states of systems of a huge number of particles in interaction. In particular, our main message is that although the historical approach based on potentials has been rather successful from a physical point of view, one has to insist on (almost sure) continuity properties of conditional probabilities to get a proper mathematical framework.

Gibbs measures are "probably" the central object of Equilibrium statistical mechanics, a branch of probability theory that takes its origin from Boltzmann ([10], 1876) and Gibbs ([51], 1902), who introduced a statistical approach to thermodynamics to deduce collective macroscopic behaviors from individual microscopic information. Starting from the observation that true physical systems with a very disordered microscopic structure, like gases, ferromagnets like irons etc., could present a more ordered, non-fluctuating, macroscopic behavior, they started considering the microscopic components as random variables and macroscopic equilibrium states as probability measures concentrating on the "most probable" states among the possible "configurations" of the microscopic system, in a sense consistent with the laws of thermodynamics. Of course, they did not use these modern probabilistic terms at that time, and it is one of the tasks of mathematical statistical mechanics to translate their intuitions in a more modern and rigorous formalism.

These ideas have been first introduced and justified by Boltzmann in its introduction of statistical entropy [11] and have been thereafter used by Gibbs as a postulate to introduce his microcanonical, canonical and grand 
canonical ensembles [51]. These Gibbs ensembles provide three different ways of describing equilibrium states, which would nowadays be called "probability measures", at the macroscopic level. The main goal of modern mathematical statistical mechanics is thus to describe rigorously these concepts in the standard framework of probability and measure theory that has been developed during the century following Boltzmann's ideas, pursuing two main goals: To describe these ensembles as proper probability measures allowing the modelization of phase transitions phenomena, and to interpret them as equilibrium states in a probabilistic sense that would incorporate ideas taken from the second law of thermodynamics.

For this purpose of describing phase transitions phenomena, roughly seen as the possibility to get different macroscopic structures for the same microscopic interaction (e.g. gas versus liquid, positive or negative magnetization of iron, etc.), we shall see that an infinite-volume formalism, which can be loosely justified by the large number of microscopic components in any macroscopic part of interacting systems, is required. For the sake of simplicity, and because it already incorporates many of the most interesting features of the theory, we shall focus on systems where the whole space is modelled by a discrete infinite lattice (mainly $\mathbb{Z}^{d}$ ), with, attached at each site, a microscopic element modelled by a finite ${ }^{1}$ value (e.g. +1 for a positive "microscopic magnetization" in iron).

To describe equilibrium states and to model phase transitions phenomena in such a framework, we are led to construct probability measures on an infinite product probability space in an alternative way to the standard Kolmogorov's construction. This alternative "DLR" construction, rigorously introduced in the late sixties by Dobrushin [27] and Lanford/Ruelle [81], makes use of systems of compatible conditional probabilities with respect to the outside of finite subsets, when the outside is fixed in a boundary condition, to reach thereafter infinite-volume quantities. This DLR approach can also be seen as an extension of the Markov chains formalism and to describe Gibbs measures we shall focus on quasilocality, a topological property of conditional probability measures which extends the Markov property.

As we shall see, this approach allows to model phase transitions and the related critical phenomena. In that case, a qualitative change of the macroscopic system at a "critical point" is physically observed, together with a very chaotic critical behavior. This criticality is physically interpreted as a highly correlated system without any "proper scale", namely where a physical quantity called the correlation length should diverge, and such a system should be thus reasonably scaling-invariant. These considerations have led to the use of the so-called Renormalization Group ( $R G)$ transformations,

\footnotetext{
${ }^{1}$ The state-space will be finite except in a very few explicitly emphasized occasions, where it would be compact or unbounded.
} 
which appeared to be a very powerful tool in the theoretical physics of critical phenomena $[17,50]$. It also gave rise to ill-understood phenomena, the $R G$ pathologies, detected in the early seventies by Griffiths/Pearce [56] and Israel [63], and interpreted a few decades later by van Enter et al. [36] as the manifestation of the occurrence of non-Gibbsianness. This last observation was the starting point of the Dobrushin program of restoration of Gibbsianness, launched by Dobrushin in 1995 in a talk in Renkum [30] and consisting in two main goals: Firstly, to provide alternative (weaker) definitions of Gibbs measures that would be stable under the natural scaling transformations of the RG, and secondly to restore the thermodynamics properties for these new notions in order to still be able to interpret them as equilibrium states. This gave rise to generalized Gibbs measures.

These notes are organized as follows: We introduce in Chapter 2 the necessary mathematical background, focusing on topological and measurable properties of functions and measures on an infinite product probability space; we also recall there important properties of conditional expectations and introduce regular versions of conditional probabilities to describe the DLR construction of measures on infinite probability product spaces, mainly following $[43,52]$. We describe then the general structure of the set of DLR measures in the realm of convexity theory and mention a few general consequences and examples at the end of the same chapter. We introduce Gibbs measures in the context of quasilocality and describe the main features of the set of Gibbs measures for a given interacting system in Chapter 3. The interpretation of Gibbs and quasilocal measures as equilibrium states is rigorously established in a general set-up in Chapter 4, and we describe RG pathologies and generalized Gibbs measures in Chapter 5. 



\section{Chapter 2}

\section{Topology and measures on product spaces}

\subsection{Configuration space: set-up and nota- tions}

\subsubsection{Lattices}

For the sake of simplicity, also loosely justified by the very discrete nature of physics, the physical space will be modelled by a lattice $S$, which in our examples will mostly be the $d$-dimensional regular lattice $\mathbb{Z}^{d}$. It is endowed with a canonical distance $d$ and its elements, called sites, will be denoted by Latin letters $i, j, x, y$, etc. A pair of sites $\{i, j\}$ such that $d(i, j)=1$ will be called nearest neighbor (n.n.) and denoted by $\langle i j\rangle$. Finite subsets of the lattice $S$ will play an important role for us and will be generically denoted by capital Greek letters $\Lambda, \Lambda^{\prime}, \Delta$, etc. We denote the set of these finite subsets of $S$ by

$$
\mathcal{S}=\{\Lambda \in \mathcal{S},|\Lambda|<\infty\}=\{\Lambda \subset \subset S\}
$$

where $|\Lambda|$ denotes the cardinality of $\Lambda$ and $\subset \subset$ means inclusion of a finite set in a bigger set. This notation $|\cdot|$ will be used for many different purposes without giving its exact meaning when it is obvious. It will be moreover mostly sufficient to work with increasing sequences $\left(\Lambda_{n}\right)_{n \in \mathbb{N}}$ of cubes, defined e.g. when the lattice is $\mathbb{Z}^{d}$ by $\Lambda_{n}=[-n, n]^{d} \cap S$, for all $n \in \mathbb{N}$. 


\subsubsection{Single-spin state-space}

To each (microscopic) site $i$ of the lattice we attach the same finite ${ }^{1}$ measurable space $\left(E, \mathcal{E}, \rho_{0}\right)$, of cardinality $e:=|E|$, sometimes called singlespin state-space or more briefly state-space. The a priori measure $\rho_{0}$ will then be chosen to be the normalized uniform counting measure on the $\sigma$-algebra $\mathcal{E}=\mathcal{P}(E)$, formally defined in terms of Dirac measures by $\rho_{0}=\frac{1}{e} \sum_{q \in E} \delta_{q}$. In our guiding example, the Ising model of ferromagnetism $[71,103]$, this set is $E=\{-1,+1\}$, but other models might be considered.

At each site $i$ of the lattice will be thus attached a random variable $\sigma_{i} \in E$, called spin to keep in mind this seminal Ising model. This finite measurable space $\mathrm{E}$ is called the single-spin state space and endowed with the discrete topology, for which the singleton sets are open, so that all the subsets of $E$ are open.

\subsubsection{Configuration space}

The microscopic states are then represented by the collections of random variables $\sigma=\left(\sigma_{i}\right)_{i \in S}$, living in the infinite product space $(\Omega, \mathcal{F}, \rho):=$ $\left(E^{S}, \mathcal{E}^{\otimes S}, \rho_{0}^{\otimes S}\right)$ called the configuration space. (Infinite-volume) configurations will be denoted by Greek letters $\sigma, \omega$, etc.

For any $\Lambda \in \mathcal{S}$, the finite product space $\Omega_{\Lambda}=E^{\Lambda}$ comes with a finite collection of the random variables $\sigma_{i}$ for the sites $i \in \Lambda$ and for any $\sigma \in \Omega$, one denotes by $\sigma_{\Lambda}=\left(\sigma_{i}\right)_{i \in \Lambda}$ this configuration at finite volume $\Lambda$. We also define concatenated configurations at infinite-volume by prescribing values on partitions of $S$, writing e.g. $\sigma_{\Lambda} \omega_{\Lambda^{c}}$ for the configuration which agrees with a configuration $\sigma$ in $\Lambda$ and with another configuration $\omega$ outside $\Lambda$.

\subsection{Measurable properties of the configura- tion space}

The product $\sigma$-algebra $\mathcal{F}=\mathcal{E}^{\otimes S}$ is the smallest $\sigma$-algebra generated by the set of cylinders $C_{\sigma_{\Delta}}=\left\{\omega \in \Omega: \omega_{\Delta}=\sigma_{\Delta}\right\}$, when $\sigma_{\Delta}$ runs over $\Omega_{\Delta}$ and $\Delta$ runs over $\mathcal{S}$. We also write $\mathcal{C}=\left\{\left(C_{\sigma_{\Delta}}\right), \sigma_{\Delta} \in \Omega_{\Delta}, \Delta \in \mathcal{S}\right\}$ and $\mathcal{C}_{\Lambda}=\left\{\left(C_{\sigma_{\Delta}}\right), \sigma_{\Delta} \in \Omega_{\Delta}, \Delta \subset \subset \Lambda\right\}$ for the family of cylinders restricted to any sub-lattice $\Lambda \subset S$, not necessarily finite. Alternatively, one defines for all sites $i$ of the lattice, the canonical projection $\Pi_{i}: \Omega \longrightarrow E$ defined for all $\omega \in \Omega$ by $\pi_{i}(\omega)=\omega_{i}$, and denotes by $\Pi_{\Lambda}$ the canonical projection

\footnotetext{
${ }^{1}$ This theory also holds, modulo a few adaptations, for more general measurable spaces, compact [52] or even non-compact [26, 86], but the simpler finite case already gets the main features of the theory. It will be our framework throughout the lectures, except in a few occasions where it will be explicitly precised.
} 
from $\Omega$ to $\Omega_{\Lambda}$ for all $\Lambda \in \mathcal{S}$, defined for $\omega \in \Omega$ by $\Pi_{\Lambda}(\omega)=\omega_{\Lambda}:=\left(\omega_{i}\right)_{i \in \Lambda}$. Then, using the following rewriting of the cylinders,

$$
C_{\sigma_{\Lambda}}=\Pi_{\Lambda}^{-1}\left(\left\{\sigma_{\Lambda}\right\}\right), \forall \sigma \in \Omega
$$

one gets that $\mathcal{F}$ is also the smallest $\sigma$-algebra that makes the projections measurable.

The macroscopic states will be represented by random fields, i.e. probability measures on $(\Omega, \mathcal{F})$, whose set will be denoted by $\mathcal{M}_{1}^{+}(\Omega, \mathcal{F})$, or more briefly $\mathcal{M}_{1}^{+}(\Omega)$. The simplest one is the a priori product measure $\rho=\rho_{0}^{\otimes S}$ defined as the product of $\rho_{0}$ on the cylinders and extended to the whole lattice by virtue of the Kolmogorov's extension theorem [7, 42], recalled later in this chapter. This particular random field models the equilibrium state of a non-interacting particle system, for which the spins are independent random variables.

In order to mathematically describe microscopic and macroscopic behaviors, one would like to distinguish local and non-local events. The local ones are the elements of a sub- $\sigma$-algebra $\mathcal{F}_{\Lambda}$ for a finite $\Lambda \in \mathcal{S}$, where $\mathcal{F}_{\Lambda}$ is the $\sigma$-algebra generated by the finite cylinders $\mathcal{C}_{\Lambda}$ defined above. A function $f: \omega \longrightarrow \mathbb{R}$ is said to be $\mathcal{F}_{\Lambda}$-measurable if and only if (iff) "it depends only on the spins in $\Lambda$ ":

$$
f \in \mathcal{F}_{\Lambda} \Longleftrightarrow\left(\omega_{\Lambda}=\sigma_{\Lambda} \Longrightarrow f(\omega)=f(\sigma)\right) .
$$

Definition 2.1 (Local functions). A function $f: \Omega \longrightarrow \mathbb{R}$ is said to be local if it is $\mathcal{F}_{\Lambda}$-measurable for some $\Lambda \in \mathcal{S}$. The set of local functions will be denoted by $\mathcal{F}_{\text {loc }}$.

We shall use the same notation $f \in \mathcal{F}$ for the measurability w.r.t a $\sigma$-algebra or $f \in \mathcal{H}$ for the membership in a space $\mathcal{H}$ of functions.

Another important sub- $\sigma$-algebra concerns macroscopic non-local events. It is the so-called $\sigma$-algebra at infinity, of tail or asymptotic events, formally defined by

$$
\mathcal{F}_{\infty}=\bigcap_{\Lambda \in \mathcal{S}} \mathcal{F}_{\Lambda^{c}}
$$

Equivalently, it is the $\sigma$-algebra (countably) generated by the tail cylinders $\mathcal{C}_{\infty}:=\cap_{\Lambda \in \mathcal{S}} \mathcal{C}_{\Lambda^{c}}$. It consists of events that do not depend on what happens in microscopic subsets of the systems; they are typically defined by some limiting procedure. In our description of the Ising model, we shall encounter for example the tail events $B_{m}$, defined, for $m \in[-1,+1]$, by

$$
B_{m}=\left\{\omega: \lim _{n \rightarrow \infty} \frac{1}{\left|\Lambda_{n}\right|} \sum_{i \in \Lambda_{n}} \omega_{i}=m\right\}
$$


that will help to distinguish the physical phases of the system. Similarly, a function $g$ is $\mathcal{F}_{\infty}$-measurable $\left(g \in \mathcal{F}_{\infty}\right)$ if it does not depend on the spins in any finite region, i.e. iff

$$
\exists \Lambda \in \mathcal{S} \text { s.t. } \sigma_{\Lambda^{\mathrm{c}}}=\omega_{\Lambda^{\mathrm{c}}} \Longrightarrow \mathrm{g}(\omega)=\mathrm{g}(\sigma) .
$$

These functions will be important later on to characterize macroscopic quantities and to detect non-Gibbsianness. They are also generally defined by some limiting procedure, the following function being e.g. tailmeasurable:

$$
\forall \omega \in \Omega, g(\omega)= \begin{cases}\lim _{n \rightarrow \infty} \frac{1}{\left|\Lambda_{n}\right|} \sum_{i \in \Lambda_{n}} \omega_{i} & \text { if the limit exists. } \\ \text { anything } & \text { otherwise. }\end{cases}
$$

Similar tail $\sigma$-algebras are also used in ergodic theory or in classical probability theory, in some 0-1-laws for example [3, 118]. To connect with these fields, we introduce here the basic notion of translation-invariance, which will also be important for physical interpretations later on. For simplicity, we introduce this notion on the lattice $S=\mathbb{Z}^{d}$ but it could be easily extended to other lattices. First, one defines translations on the lattice as a family of invertible transformations $\left(\tau_{x}\right)_{x \in \mathbb{Z}^{d}}$ indexed by the sites of the lattice and defined for all $x \in \mathbb{Z}^{d}$ by

$$
\tau_{x}: y \longmapsto \tau_{x} y=y+x \in \mathbb{Z}^{d}
$$

where additions and subtractions on the lattice are standard. They induce translations on $\Omega$ : The translate by $x$ of $\omega \in \Omega$ is the configuration $\tau_{x} \omega$ defined for all $i \in S$ by

$$
\left(\tau_{x} \omega\right)_{i}=\omega_{\tau_{-x} i}=\omega_{i-x}
$$

It also extends naturally to measurable sets (our "events"), measurable functions and measures. In particular, the set of translation-invariant probability measures on $(\Omega, \mathcal{F})$ is denoted by $\mathcal{M}_{1 \text {, inv }}^{+}(\Omega)$ and the $\sigma$-algebra generated by the translation-invariant functions is the translation-invariant $\sigma$-algebra denoted by $\mathcal{F}_{\text {inv }}$.

Let us briefly leave the field of lattices to consider another framework that links our approach to exchangeability in the context of the so-called mean-field models. When $S=\mathbb{N}$, one can define first a group $I_{n}$ of permutations at finite volume $n$, that are bijections leaving invariant the sites $i>n$, and define its union $I=\cup_{n \in \mathbb{N}} I_{n}$ to be the group of all permutations of finitely many coordinates. The $I$-invariant probability measures on $(\Omega, \mathcal{F})$ form the set $\mathcal{M}_{I}$ of exchangeable probability measures. For $n \in \mathbb{N}$, 
the $\sigma$-algebra of the events invariant under permutations of order $n$ is defined to be

$$
\mathcal{I}_{n}=\left\{A \in I_{n}: \pi^{-1} A=A, \forall \pi \in I_{n}\right\}
$$

and its intersection is the $\sigma$-algebra of symmetric or permutation-invariant events

$$
\mathcal{I}=\bigcap_{n \in \mathbb{N}} \mathcal{I}_{n}
$$

\subsection{Topological properties of the configura- tion space}

\subsubsection{Product topology}

As we shall see, the notion of Gibbs measures is based on the interplay between topology and measure theory, and to relate these notions we need to introduce a topology $\mathcal{T}$ whose Borel $\sigma$-algebra coincides with $\mathcal{F}$. The latter and $\mathcal{T}$ are then said to be compatible in the sense that both open sets and continuous functions are then measurable. Thus, the topology $\mathcal{T}$ is endowed with the same generators as those of $\mathcal{F}$ and $\mathcal{T}$ is the smallest topology on $\Omega$ containing the cylinders or making the projections continuous. To do so, we consider, on the whole configuration space $(\Omega, \mathcal{F})$ the product topology, $\mathcal{T}=\mathcal{T}_{0}^{\otimes_{S}}$ of the discrete topology $\mathcal{T}_{0}$ on $E$. Endowed with these topological and measurable structures, our configuration space has the following nice properties:

Theorem 2.4. [43, 52] The topological space $(\Omega, \mathcal{T})$ is compact, its Borel $\sigma$-algebra coincides with the product $\sigma$-algebra $\mathcal{F}$, and the measurable space $(\Omega, \mathcal{F})$ is a Polish space, i.e. metrizable, separable and complete.

Compactness follows from Tychonov's theorem and will be helpful in proving existence results and to simplify the topological characterizations of Gibbs measures. To get a metric on this compact space, one can choose $\delta: \Omega \times \Omega \longrightarrow \mathbb{R}^{+}$, defined for all $\omega, \sigma \in \Omega$ by

$$
\delta(\omega, \sigma)=\sum_{i \in S} 2^{-n(i)} \mathbf{1}_{\left\{\omega_{i} \neq \sigma_{i}\right\}}
$$

where $n: S \longrightarrow \mathbb{N}$ is any bijection assumed to be fixed and known. With this topology, open sets are finite unions of cylinders and in particular, a typical neighborhood of $\omega \in \Omega$ is given by a cylinder for $\Lambda \in \mathcal{S}$ denoted in this context by

$$
\mathcal{N}_{\Lambda}(\omega)=\left\{\sigma \in \Omega: \sigma_{\Lambda}=\omega_{\Lambda}, \sigma_{\Lambda^{c}} \text { arbitrary }\right\} .
$$

Similarly, when $S=\mathbb{Z}^{d}$, a basis of neighborhoods of a configuration $\omega \in \Omega$ is given by the family of cylinders $\left(\mathcal{N}_{\Lambda_{n}}(\omega)\right)_{n \in \mathbb{N}}$, for a sequence of cubes 
$\left(\Lambda_{n}\right)_{n \in \mathbb{N}}$. Thus, two configurations are closed in this topology if they coincide over large finite regions, and the larger the region is, the closer they $\mathrm{are}^{2}$. Moreover, the set of asymptotic events is dense for this topology, because they are insensitive to changes in finite regions. In particular, the set of configurations that are asymptotically constant is a countable and dense subset, leading thus to separability of the product topology by compatibility of the latter with the measurable structure.

\subsubsection{Quasilocality for functions}

This nice topological setting allows us to provide different equivalent characterizations of microscopic quantities. Firstly, we find it natural to say that a microscopic function $f$ on $\Omega$ is arbitrarily "close" to functions which depend on finitely many coordinates, i.e. local functions. This leads to the important concept of a quasilocal function:

Definition 2.5. A function $f: \Omega \longrightarrow \mathbb{R}$ is said to be quasilocal if it can be uniformly approximated by local functions, i.e. if for each $\epsilon>0$, there exists $f_{\epsilon} \in \mathcal{F}_{\text {loc }}$ s.t.

$$
\sup _{\omega \in \Omega}\left|f(\omega)-f_{\epsilon}(\omega)\right|<\epsilon .
$$

We denote by $\mathcal{F}_{\text {qloc }}$ the set of quasilocal functions. It is the uniform closure of $\mathcal{F}_{\text {loc }}$ in the sup-norm, and by compactness is automatically bounded. Moreover, due to the Polish and compact structure of $\Omega$, one can use sequences and continuity coincides with uniform continuity. Quasilocal functions are continuous while (non-constant) asymptotic tail-measurable functions are discontinuous. Then, using the metric $\delta$ or the basis of neighborhoods described above, it is a simple exercise to prove that quasilocal functions are in fact the (uniformly) continuous functions on $\Omega$, and we use it in the next lemma to give alternative definitions of quasilocality. When we do not use sequences, we shall deal with the following convergence:

\section{Definition 2.6 (Convergence along a net directed by inclusion).}

$$
\lim _{\Lambda \uparrow \mathcal{S}} F(\Lambda)=a
$$

means convergence of a set-function $F: \mathcal{S} \longrightarrow \mathbb{R}$ along a set $\mathcal{S}$ directed by inclusion:

$$
\forall \epsilon>0, \exists K_{\epsilon} \in \mathcal{S} \text { s.t. } \mathcal{S} \ni \Lambda \supset K_{\epsilon} \Longrightarrow|F(\Lambda)-a| \leq \epsilon .
$$

Thus, in our settings when the single-spin space is finite, one has the following

\footnotetext{
${ }^{2}$ This topological framework is standard also when one consider Cantor sets and dyadic expansions of reals.
} 
Lemma 2.7. [43, 52] A function $f: \Omega \longrightarrow \mathbb{R}$ is quasilocal iff one of the following holds:

- Continuity: It is continuous at every $\omega \in \Omega$, i.e. $\forall \omega \in \Omega, \forall \epsilon>0$, $\exists n \in \mathbb{N}$ s.t.

$$
\sup _{\sigma \in \Omega}\left|f\left(\omega_{\Lambda_{n}} \sigma_{\Lambda_{n}^{c}}\right)-f(\omega)\right|<\epsilon .
$$

- Uniform limit of local functions: There exists $\left(f_{n}\right)_{n \in \mathbb{N}}$ s.t. for all $n \in \mathbb{N}, f_{n} \in \mathcal{F}_{\Lambda_{n}}$ and

$$
\lim _{n \rightarrow \infty} \sup _{\omega \in \Omega}\left|f_{n}(\omega)-f(\omega)\right|=0 .
$$

- Sequential uniform continuity: $\forall \epsilon>0$, there exists $n \in \mathbb{N}$ s.t.

$$
\sup _{\sigma, \omega \in \Omega}\left|f\left(\omega_{\Lambda_{n}} \sigma_{\Lambda_{n}^{c}}\right)-f(\omega)\right|<\epsilon
$$

- Uniform continuity:

$$
\lim _{\Lambda \uparrow \mathcal{S}} \sup _{\omega, \sigma \in \Omega, \omega_{\Lambda}=\sigma_{\Lambda}}|f(\omega)-f(\sigma)|=0 .
$$

An important consequence of this lemma is that a non-constant tailmeasurable function can never be quasilocal. For example, let us consider the event

$$
B_{0}=\left\{\omega: \lim _{n \rightarrow \infty} \frac{1}{\left|\Lambda_{n}\right|} \sum_{i \in \Lambda_{n}} \omega_{i}=0\right\} .
$$

The indicator function $f$ of this event is tail-measurable, non-constant and non-quasilocal. Take for example $\Omega=\{0,1\}^{\mathbb{Z}}$. The configuration $\omega=\mathbf{0}$, null everywhere, belongs to $B_{0}$ and $f(\mathbf{0})=1$. Let $\mathcal{N}$ be a neighborhood of this null configuration, and choose it to be $\mathcal{N}_{\Lambda_{n}}(\mathbf{0})$ for some $n>0$. There exists then $\sigma \in \mathcal{N}_{\Lambda_{n}}(\mathbf{0})$ such that $\sigma_{\Lambda_{n}^{c}}=\mathbf{1}_{\Lambda_{n}^{c}}$, where $\mathbf{1} \in \Omega$ is the configuration which value is 1 everywhere. For this configuration, $\lim _{n \rightarrow \infty} \frac{1}{\left|\Lambda_{n}\right|} \sum_{i \in \Lambda_{n}} \sigma_{i}=1$ and thus $f(\sigma)=0$ : This proves that $f$ is discontinuous and thus non-quasilocal. Non-quasilocal functions will be important to detect non-Gibbsian measures in Chapter 5.

\subsubsection{Weak convergence of probability measures}

We have already introduced the space $\mathcal{M}_{1}^{+}(\Omega, \mathcal{F})$ of probability measures on $(\Omega, \mathcal{F})$ that represents the macroscopic possible states of our systems. Before introducing different ways of constructing such measures on our infinite product spaces, we need a proper notion for the convergence of 
probability measures, i.e. to introduce a topology on $\mathcal{M}_{1}^{+}(\Omega)$. A strong way to do so is to consider the topology inherited from the so-called totalvariation norm but this is indeed too strong a notion of convergence for the sake of describing "non-chaotic" equilibrium states: Physically, this convergence means that expected values converges, uniformly for all bounded or continuous observables, i.e. microscopic in our point of view, and this occurs rarely in physical situations. We shall thus require a topology whose convergence mainly concerns non-uniform expectations of microscopic variables. This is the famous weak convergence of probability measures, which is indeed weaker than most ways of convergence, see [7, 42, 118]. It focus on convergences of expectations of continuous functions and for any $\mu \in \mathcal{M}_{1}^{+}(\Omega)$ we write $\mu[f]=\int f d \mu$ for the expectation under $\mu$ of any bounded function $f$.

Definition 2.8 (Weak convergence). A sequence $\left(\mu_{n}\right)_{n \in \mathbb{N}}$ in $\mathcal{M}_{1}^{+}(\Omega, \mathcal{F})$ is said to converge weakly to $\mu \in \mathcal{M}_{1}^{+}(\Omega, \mathcal{F})$ if expectations of continuous functions converge:

$$
\mu_{n} \stackrel{W}{\longrightarrow} \mu \Longleftrightarrow \lim _{n \rightarrow \infty} \mu_{n}[f]=\mu[f], \forall f \in \mathcal{F}_{\text {qloc }}
$$

This convergence gives no information on the convergence of the expectations of discontinuous (macroscopic, asymptotic) quantities. This will be important for our purpose of modelling phase transitions phenomena by working at finite but larger and larger sets through some infinite-volume limit. By definition, the set of local functions is dense in $\mathcal{F}_{\mathrm{qloc}}$, so it is enough to test this convergence on $\mathcal{F}_{\text {loc }}$ or on cylinders.

To describe, at the end of the chapter, the general convex structure of the set of Gibbs measures in case of phase transitions, we shall also need to deal with probability measures on spaces of probability measures, and we first endow such spaces with a canonical measurable structure. For any subset of probability measures $\mathcal{M} \subset \mathcal{M}_{1}^{+}(\Omega, \mathcal{F})$, the natural way to do so is to evaluate any $\mu \in \mathcal{M}$ via the numbers $\{\mu(A), A \in \mathcal{F}\}$. One introduces then the evaluation maps on $\mathcal{M}$ defined for all $A \in \mathcal{F}$ by

$$
e_{A}: \mathcal{M} \longrightarrow[0,1] ; \mu \longmapsto e_{A}(\mu)=\mu(A) \text {. }
$$

The evaluation $\sigma$-algebra $e(\mathcal{M})$ is then the smallest $\sigma$-algebra on $\mathcal{M}$ that makes measurable these evaluation maps, or equivalently the $\sigma$-algebra generated by the sets $\left\{e_{A} \leq c\right\}$ for all $A \in \mathcal{F}, 0 \leq c \leq 1$. For any bounded measurable function $f \in \mathcal{F}$, the map

$$
e_{f}: \mathcal{M} \longrightarrow \mathbb{R} ; \mu \longmapsto e_{f}(\mu):=\mu[f]
$$

is then $e(\mathcal{M})$-measurable. 


\subsection{Probability theory on infinite product spaces}

\subsubsection{Kolmogorov's consistency}

The standard way to construct probability measures on an infinite product measurable space is to start from a consistent system of finite dimensional marginals, following a terminology of Kolmogorov [7]:

Definition 2.10. A family $\left(\mu_{\Lambda}\right)_{\Lambda \in \mathcal{S}}$ of probability measures on $\left(\Omega_{\Lambda}, \mathcal{F}_{\Lambda}\right)$ is said to be consistent in the sense of Kolmogorov iff for all $\Lambda \subset \Lambda^{\prime} \in$ $\mathcal{S}, \forall A \in \mathcal{F}_{\Lambda}$,

$$
\mu_{\Lambda}(A)=\mu_{\Lambda^{\prime}}\left(\left(\Pi_{\Lambda}^{\Lambda^{\prime}}\right)^{-1}(A)\right)
$$

where $\Pi_{\Lambda}^{\Lambda^{\prime}}$ is the natural projection from $\Omega_{\Lambda^{\prime}}$ to $\Omega_{\Lambda}$. Equivalently, this is equivalent to require for all $\Lambda \subset \Lambda^{\prime} \in \mathcal{S}$ and $B \in \mathcal{F}$ that

$$
\mu_{\Lambda}\left(\Pi_{\Lambda}(B)\right)=\mu_{\Lambda^{\prime}}\left(\Pi_{\Lambda^{\prime}}(B)\right)
$$

We have seen that when the single-spin state-space is finite, it is also a Polish space, and given a consistent family of conditional probabilities, it is possible to extend it to the whole configuration space, thanks to the following

Theorem 2.11 (Kolmogorov's extension theorem). Let $\left(\mu_{\Lambda}\right)_{\Lambda \in \mathcal{S}}$ be a consistent family of marginal distributions on a Polish infinite-product probability space $(\Omega, \mathcal{F})$. Then there exists a unique probability measure $\mu \in \mathcal{M}_{1}^{+}(\Omega)$ s.t. for all $\Lambda \in \mathcal{S}, \forall A \in \mathcal{F}_{\Lambda}$,

$$
\mu\left(\Pi_{\Lambda}^{-1}(A)\right)=\mu(A)
$$

where $\Pi_{\Lambda}^{-1}(A)$ is the pre-image of $A$ by the projection from $\Omega$ to $\Omega_{\Lambda}$, defined by

$$
\Pi_{\Lambda}^{-1}(A)=\left\{\sigma \in \Omega: \Pi_{\Lambda}(\sigma)=\sigma_{\Lambda} \in A\right\} .
$$

Equivalently, this is also the unique probability measure on $(\Omega, \mathcal{F})$ such that

$$
\forall B \in \mathcal{F}, \mu(B)=\mu_{\Lambda}\left(\Pi_{\Lambda}(B)\right)
$$

The main example of application of this theorem is the construction of the a priori product measure $\rho$ on $(E, \mathcal{E})$. Consider the counting measure $\rho_{0} \in \mathcal{M}_{1}^{+}(\Omega)$ on the single-site state space and the finite product measure $\rho_{\Lambda}=\rho_{0}^{\otimes \Lambda}$ on any of the finite product probability spaces $\left(\Omega_{\Lambda}, \mathcal{F}_{\Lambda}\right)$, defined for all $\Lambda \in \mathcal{S}$ on the cylinders by

$$
\rho_{\Lambda}\left(\sigma_{\Lambda}\right)=\prod_{i \in \Lambda} \rho_{0}(i), \forall \sigma_{\Lambda} \in \Omega_{\Lambda}
$$


and extended on $\mathcal{F}_{\Lambda}$ by requiring, for all $A \in \mathcal{F}_{\Lambda}$,

$$
\rho_{\Lambda}(A)=\sum_{\sigma_{\Lambda} \in A} \rho_{\Lambda}\left(\sigma_{\Lambda}\right) .
$$

The system $\left(\rho_{\Lambda}\right)_{\Lambda \in \mathcal{S}}$ is trivially a consistent family of marginals which can thus be extended into a unique probability measure $\rho:=\rho_{0}^{\otimes S}$.

Hence, we know how to build elements $\mu$ of the (convex) set $\mathcal{M}_{1}^{+}(\Omega, \mathcal{F})$, that are thus interpreted as macroscopical descriptions of the physical phases of the systems in our settings. The problem now is that we also want to model phase transitions, i.e. to get different infinite-volume measures corresponding to the same finite-volume description, and to do so we have to proceed differently and work with systems of conditional probabilities consistent in a different sense than that of Kolmogorov. Before describing this so-called DLR approach, based on successive conditionings w.r.t. decreasing sub- $\sigma$-algebras, we first recall a few important properties of conditional probabilities on infinite product probability Polish spaces.

\subsubsection{Regular versions of conditional probabilities}

Definition 2.12. [Conditional expectation] Let $(\Omega, \mathcal{F})$ be a measurable space, $\mu \in \mathcal{M}_{1}^{+}(\Omega, \mathcal{F}), \mathcal{G}$ a sub- $\sigma$-algebra of $\mathcal{F}$ and $f \in \mathcal{F}, \mu$-integrable. $A$ conditional expectation of $f$ given $\mathcal{G}$, w.r.t. $\mu$, is a measurable function $\mathbb{E}_{\mu}[f \mid \mathcal{G}]: \Omega \longrightarrow \mathbb{R} ; \omega \longmapsto \mathbb{E}_{\mu}[f \mid \mathcal{G}](\omega)$ such that

1. $\mathbb{E}_{\mu}[f \mid \mathcal{G}]$ is $\mathcal{G}$-measurable.

2. For any $g \in \mathcal{G}$ bounded, $\quad \int g \cdot \mathbb{E}_{\mu}[f \mid \mathcal{G}] d \mu=\int g \cdot f d \mu$.

$$
\text { In particular } \quad \int \mathbb{E}_{\mu}[f \mid \mathcal{G}] d \mu=\int f d \mu .
$$

The existence of such functions is ensured by the Radon-Nikodým theorem $[101,118]$; because a $\mu$-integral being involved in point 2 . of the definition above, such a conditional expectation is not unique, but two different versions of it can only differ at most on a set of $\mu$-measure zero. So, Definition 2.12 does not define a unique function, but measure-zero modifications are however the only ones possible: The conditional expectation $\mathbb{E}_{\mu}[f \mid \mathcal{G}]$ is thus defined " $\mu$-a.s." and, as a major consequence, its extension to define conditional probabilities that would be effectively probability measures cannot be straightforward. Indeed, the good candidate for such an almost-surely defined conditional probability $\mu_{\mathcal{G}}(\cdot \mid \omega)$ would be, for all $A \in \mathcal{F}$,

$$
\mu_{\mathcal{G}}(A \mid \omega):=\mathbb{E}_{\mu}\left[\mathbf{1}_{A} \mid \mathcal{G}\right](\omega), \quad \mu-\text { a.s. }(\omega) .
$$


but one has to be careful because the following characterizing properties of a probability measure are true almost-surely only:

- $\mu_{\mathcal{G}}(\Omega \mid \cdot)=1, \mu$-a.s. and $\mu_{\mathcal{G}}(\emptyset \mid \cdot)=0, \mu$-a.s.

- For all $A \in \mathcal{F}, 0 \leq \mu_{\mathcal{G}}(A \mid \cdot) \leq 1, \mu$-a.s.

- For any countable collection $\left(A_{i}\right)_{i \in I}$ of pairwise disjoints $\mathcal{F}$-measurable sets,

$$
\mu_{\mathcal{G}}\left(\cup_{i} A_{i} \mid \cdot\right)=\sum_{i} \mu_{\mathcal{G}}\left(A_{i} \mid \cdot\right), \mu-\text { a.s. }
$$

and one also has that for all $B \in \mathcal{G}, \mu_{\mathcal{G}}(B \mid \cdot)=\mathbf{1}_{B}(\cdot)$, $\mu$-a.s.

The problem in this definition comes from the fact that the sets of measure zero that appear in the former characterizing properties depend on the sets $A$ and $\left(A_{i}\right)_{i \in I} \in \mathcal{F}$ considered; the later being uncountably many, we cannot say that we have defined these conditional probabilities $\mu$-almost everywhere ${ }^{3}$. What is needed to say so is to get a unique set of $\mu$-measure zero, independent of the sets $A$ and $\left(A_{i}\right)_{i \in I}$, outside which the above properties are true. In such a case, one says that there exists $a$ regular version of the conditional probabilities of $\mu$ w.r.t. sub- $\sigma$-algebras of $\mathcal{F}$. More precisely, this occurs when there exists a probability kernel (see next subsection) $\mu_{\mathcal{G}}$ from $(\Omega, \mathcal{F})$ to itself such that

$$
\mu \text {-a.s., } \mu_{\mathcal{G}}[f \mid \cdot]=\mathbb{E}_{\mu}[f \mid \mathcal{G}](\cdot), \forall f \in \mathcal{F} \text { bounded }
$$

where the " $\mu$-a.s." means that there exists a (mostly abstract) measurable set of full $\mu$-measure $\Omega_{\mu}$ where the above characterizing properties of a probability measure hold for all $\omega \in \Omega_{\mu}$, independently of the measurable set $A \in \mathcal{F}$. In our framework, this is fortunately granted:

Theorem 2.13. [101] Any probability measure on a Polish probability space $(\Omega, \mathcal{F})$ admits a regular conditional probability w.r.t. any sub- $\sigma-$ algebra of $\mathcal{F}$.

We also mention here two direct consequences of Definition 2.12 which will be useful to characterize measures in terms of systems of regular conditional probabilities. Keeping the same settings, one has, $\mu$-almost surely, for any bounded $\mathcal{G}$-measurable function $g$, any bounded measurable function $f$ and any sub- $\sigma$-algebra $\mathcal{G}^{\prime} \subset \mathcal{G}$,

$$
\mu_{\mathcal{G}}[g \cdot f \mid \cdot]=\mathbb{E}_{\mu}[g \cdot f \mid \mathcal{G}](\cdot)=g \cdot \mathbb{E}_{\mu}[f \mid \mathcal{G}]=g \cdot \mu_{\mathcal{G}}[f \mid \cdot]
$$

and

$$
\mu_{\mathcal{G}^{\prime}}\left[\mu_{\mathcal{G}}[f \mid \cdot] \cdot\right]=\mathbb{E}_{\mu}\left[\mathbb{E}_{\mu}[f \mid \mathcal{G}] \mid \mathcal{G}^{\prime}\right](\cdot)=\mathbb{E}_{\mu}\left[f \mid \mathcal{G}^{\prime}\right](\cdot)=\mu_{\mathcal{G}^{\prime}}[f \mid \cdot] .
$$

\footnotetext{
${ }^{3}$ To see how to construct counter-examples, consult e.g. [115].
} 
We recall now the useful concept of probability kernel to describe the alternative way of defining probability measures on infinite product probability spaces introduced in the late sixties by Dobrushin, Lanford and Ruelle to model phase transitions.

Definition 2.15. A probability kernel from a probability space $(\Omega, \mathcal{F})$ to a probability space $\left(\Omega^{\prime}, \mathcal{F}^{\prime}\right)$ is a map $\gamma(\cdot \mid \cdot): \mathcal{F}^{\prime} \times \Omega \rightarrow[0,1]$ such that

- For all $\omega \in \Omega, \gamma(\cdot \mid \omega)$ is a probability measure on $\left(\Omega^{\prime}, \mathcal{F}^{\prime}\right)$.

- For all $A^{\prime} \in \mathcal{F}^{\prime}, \gamma\left(A^{\prime} \mid \cdot\right)$ is $\mathcal{F}$-measurable.

The simplest example is the map $\gamma(A \mid \omega)=\mathbf{1}_{A}(\omega)$ defined for any probability space $(\Omega, \mathcal{F})$, any $A \in \mathcal{F}$, any $\omega \in \Omega$. It is a probability kernel from $(\Omega, \mathcal{F})$ into itself. More interesting examples concern regular versions of conditional probabilities, Markov transition kernels etc. We extend this notion in order to introduce the concept of specification and to prescribe conditional probabilities of a measure to try to define it. To do so, we state a few definitions.

Definition 2.16. Let $\gamma$ be a probability kernel from $(\Omega, \mathcal{F})$ to $\left(\Omega^{\prime}, \mathcal{F}^{\prime}\right)$. For any function $f \in \mathcal{F}^{\prime}$, we define $\gamma f \in \mathcal{F}$ to be the function defined for all $\omega \in \Omega$ by

$$
\gamma f(\omega)=\int_{\Omega^{\prime}} f(\sigma) \gamma(d \sigma \mid \omega)
$$

We also define for any $\mu \in \mathcal{M}_{1}^{+}(\Omega, \mathcal{F})$ the measure $\mu \gamma \in \mathcal{M}_{1}^{+}\left(\Omega^{\prime}, \mathcal{F}^{\prime}\right)$ by

$$
\forall A^{\prime} \in \mathcal{F}^{\prime}, \quad \mu \gamma\left(A^{\prime}\right)=\int_{\Omega} \gamma\left(A^{\prime} \mid \omega\right) \mu(d \omega) .
$$

A little bit more has to be required for a kernel to represent a regular version of a conditional probability. In order to illustrate the "doubleconditioning" stability (2.14) of conditional probabilities we also introduce the notion of product (or composition) of kernels.

Definition 2.17. Let $\gamma$ be a kernel from $(\Omega, \mathcal{F})$ to $\left(\Omega^{\prime}, \mathcal{F}^{\prime}\right)$ and $\gamma^{\prime}$ a kernel from $\left(\Omega^{\prime}, \mathcal{F}^{\prime}\right)$ to $\left(\Omega^{\prime \prime}, \mathcal{F}^{\prime \prime}\right)$. Then the product $\gamma \gamma^{\prime}$ is the kernel from $(\Omega, \mathcal{F})$ to $\left(\Omega^{\prime \prime}, \mathcal{F}^{\prime \prime}\right)$ s.t.

$$
\forall A^{\prime \prime} \in \mathcal{F}^{\prime \prime}, \forall \omega \in \Omega, \quad \gamma \gamma^{\prime}\left(A^{\prime \prime} \mid \omega\right)=\int_{\Omega^{\prime}} \gamma^{\prime}\left(A^{\prime \prime} \mid \sigma\right) \gamma(d \sigma \mid \omega) .
$$

We are now ready to give the more formal

Definition 2.18 (Regular version of conditional probability). Let $(\Omega, \mathcal{F}, \mu)$ be a probability space and $\mathcal{G}$ a sub- $\sigma$-algebra of $\mathcal{F}$. A regular (version of) conditional probability of $\mu$ given $\mathcal{G}$ is a probability kernel $\mu_{\mathcal{G}}(\cdot \mid \cdot)$ from $(\Omega, \mathcal{G})$ to $(\Omega, \mathcal{F})$ s.t.

$$
\mu \text {-a.s., } \quad \mu_{\mathcal{G}}[f \mid \cdot]=\mathbb{E}_{\mu}[f \mid \mathcal{G}](\cdot), \forall f \in \mathcal{F} \text { and } \mu \text {-integrable }
$$


Using the action of a kernel to a measure and the definition of the conditional expectation, it is also possible to characterize it in a more closed form, which will lead soon to a consistency condition different from the one of Kolmogorov.

Proposition 2.19 (Regular conditional probability II). In the same settings as above, a regular conditional probability of $\mu$ given $\mathcal{G}$ is a probability kernel $\mu_{\mathcal{G}}(\cdot \mid \cdot)$ from $(\Omega, \mathcal{F})$ to itself s.t. for all $f \mathcal{F}$-measurable and $\mu$-integrable,

1. $\mu_{\mathcal{G}}[f \mid \cdot]$ is $\mathcal{G}$-measurable.

2. $\mu$-a.s., $\mu_{\mathcal{G}}[g \cdot f \mid \cdot]=g \cdot \mu_{\mathcal{G}}[f \mid \cdot]$, for each bounded $g \in \mathcal{G}$

3. The kernel leaves invariant the probability measure $\mu$ : $\quad \mu \mu_{\mathcal{G}}=\mu$.

Coupled to the fact that every probability measure on a Polish space has regular conditional probabilities, Proposition 2.19 enables also to describe the double conditioning property in terms of kernels and will give rise to the concept of specification.

Definition 2.20 (System of regular conditional probabilities). Let $(\Omega, \mathcal{F}, \mu)$ be a probability space and $\left(\mathcal{F}_{i}\right)_{i \in I}$ a family of sub- $\sigma$-algebras of $\mathcal{F}$. A system of regular conditional probabilities of $\mu$ given $\left(\mathcal{F}_{i}\right)_{i \in I}$ is a family of probability kernels $\left(\mu_{\mathcal{F}_{i}}\right)_{i \in I}$ on $(\Omega, \mathcal{F})$ s.t.

1. For each $i \in I, \mu_{\mathcal{F}_{i}}$ is a regular conditional probability of $\mu$ given $\mathcal{F}_{i}$.

2. If $i, j \in I$ are such that $\mathcal{F}_{i} \subset \mathcal{F}_{j}$, then $\mu_{\mathcal{F}_{i}} \mu_{\mathcal{F}_{j}}=\mu_{\mathcal{F}_{i}}, \mu$-a.s.

In statistical mechanics we go in the opposite direction: Starting from a regular system of conditional probabilities, one wants to reconstruct probability measures, and so one aims at removing the " $\mu$-a.s." of the last definition, as one wants to obtain the same conditional probabilities for different measures.

\subsubsection{DLR-consistency and specifications}

Around 1970, Dobrushin [27], Lanford/Ruelle [81] have introduced a new way to construct probability measures on infinite product probability spaces that does not immediately yield uniqueness in the case of a Polish space, leaving the door open to the modelling of phase transitions in mathematical statistical mechanics. The key-point of their approach is to replace a system of marginals consistent in the sense of Kolmogorov by a system of regular conditional probabilities with respect to the outside of any finite set, giving rise to finite-volume versions of conditional probabilities with prescribed boundary condition(s). 
Let us consider now $\Lambda^{\prime} \subset \Lambda \in \mathcal{S}$ and the family of sub- $\sigma$-algebras $\left(\mathcal{F}_{\Lambda^{c}}\right)_{\Lambda \in \mathcal{S}}$, directed by inclusion in the sense that if $\Lambda^{\prime} \subset \Lambda \in \mathcal{S}$, one has

$$
\mathcal{F}_{\Lambda^{c}} \subset \mathcal{F}_{\Lambda^{\prime c}} \text { and } \bigcap_{\Lambda \in \mathcal{S}} \mathcal{F}_{\Lambda^{c}}=\mathcal{F}_{\infty}
$$

A system of regular conditional probabilities of $\mu \in \mathcal{M}_{1}^{+}(\Omega)$ w.r.t. the mentioned filtration exists, according to preceding section. To remove the " $\mu$-a.s" dependency and describe candidates to represent this system in the case of an equilibrium state, Preston [107] has introduced the concept of specification.

Definition 2.21 (Specification). A specification is a family $\gamma=\left(\gamma_{\Lambda}\right)_{\Lambda \in \mathcal{S}}$ of probability kernels from $(\Omega, \mathcal{F})$ into itself such that

1. For all $A \in \mathcal{F}, \gamma_{\Lambda}(A \mid \cdot)$ is $\mathcal{F}_{\Lambda^{c}}$-measurable.

2. (Properness) For all $\omega \in \Omega, B \in \mathcal{F}_{\Lambda^{c}}, \gamma_{\Lambda}(B \mid \omega)=\mathbf{1}_{B}(\omega)$

3. (Consistency)

$$
\Lambda^{\prime} \subset \Lambda \in \mathcal{S} \Longrightarrow \gamma_{\Lambda} \gamma_{\Lambda^{\prime}}=\gamma_{\Lambda}
$$

We recall that $\gamma_{\Lambda} \gamma_{\Lambda^{\prime}}$ is the map on $\Omega \times \mathcal{F}$ defined by

$$
\gamma_{\Lambda} \gamma_{\Lambda^{\prime}}(A \mid \omega)=\int_{\Omega} \gamma_{\Lambda^{\prime}}\left(A \mid \omega^{\prime}\right) \gamma_{\Lambda}\left(d \omega^{\prime} \mid \omega\right) .
$$

Specifications are thus the appropriate objects to describe conditional probabilities; an important point is that they are defined everywhere on $\Omega$, for the convenient reason that we want to deal with objects defined everywhere (not $\mu$-a.s.), and characterize $\mu$ afterwards. This will allow the description of different measures for a single specification, that is to model phase transitions in our settings. We also emphasize that for all $\sigma, \omega \in \Omega$, for all $\Lambda \in \mathcal{S}, \gamma_{\Lambda}(\sigma \mid \omega)$ depends only on $\sigma_{\Lambda}$ and $\omega_{\Lambda^{c}}$. For this reason, because only the components of $\omega$ outside $\Lambda$ (beyond the boundary) are involved, $\omega$ is often called boundary condition in statistical mechanics, and we shall use this term frequently in next sections. Moreover, conditions 1. and 2. of the definition of a specification can be removed by requiring $\gamma_{\Lambda}$ to be kernels from $\left(\Omega_{\Lambda^{c}}, \mathcal{F}_{\Lambda^{c}}\right)$ to $\left(\Omega_{\Lambda}, \mathcal{F}_{\Lambda}\right)$.

In fact, our main goal in this course is precisely to describe the set the measures satisfying the consistency relation when $\Lambda$ becomes the whole lattice.

Definition 2.23 (DLR measures). Let $\gamma$ be a specification on $(\Omega, \mathcal{F})$. The set of DLR measures for $\gamma$ is the set

$\mathcal{G}(\gamma)=\left\{\mu \in \mathcal{M}_{1}^{+}(\Omega): \forall \Lambda \in \mathcal{S}, \mu\left[A \mid \mathcal{F}_{\Lambda^{c}}\right](\cdot)=\gamma_{\Lambda}(A \mid \cdot), \mu\right.$-a.s., $\left.\forall A \in \mathcal{F}\right\}$ 
of the probability measures consistent with $\gamma$. Equivalently,

$$
\mu \in \mathcal{G}(\gamma) \Longleftrightarrow \mu \gamma_{\Lambda}=\mu, \forall \Lambda \in \mathcal{S}
$$

A DLR measure is thus a measure specified by some specification $\gamma$.

This definition contrasts with that of Kolmogorov's consistency: Instead of dealing with the family of marginals of the measure, we deal with its system of conditional probabilities. Such a DLR measure is then seen as an equilibrium state because the consistency relation implies that integrating out with respect to boundary conditions typical for the "equilibrium" DLR state outside a finite volume does not change the state in the finite volume. We shall be more precise about equilibrium properties in Chapter 4 . On a Polish space, the Kolmogorov compatibility yields existence and uniqueness of the consistent measure, whereas the set $\mathcal{G}(\gamma)$ could have a very different structure, the latter being a very important fact for our purpose of modelling the phenomenon of phase transitions. Indeed, in contrast to what occurs in Kolmogorov's consistency theorem, here neither existence, nor uniqueness needs to occur. Before describing more precisely various sets of DLR-measures, we provide a few examples describing these different possible structures.

\subsubsection{Examples}

We begin by two examples that illustrate the negative side of this description, the possibility of non-existence of measures specified by a specification. Although the first one does not lie in our settings because the singlespin state-space is not finite, the second does and helps us to extract the topological properties required to build a satisfactory framework describing Gibbs measures as equilibrium states of interacting particle systems. Thereafter, we provide an example of uniqueness (reversible Markov chain) followed by an example of non-uniqueness interpreted as the occurrence of a phase transition, the standard Ising model.

\section{One-dimensional random walk}

This analysis goes back to Spitzer but our presentation is inspired by [109]. The single-spin state-space $E=\mathbb{Z}$ is not finite nor compact so it is a bit out of our field, but we introduce it to illustrate the possibility of non-existence of any DLR measure. The lattice is the time, modelled by $S=\mathbb{Z}$. The symmetric n.n. random walk on $\mathbb{Z}, Y=\left(Y_{n}\right)_{n \in \mathbb{Z}}$, is then a random element of the configuration space $\Omega=\mathbb{Z}^{\mathbb{Z}}$ and we denote by $\mathbb{P}$ its law on $(\Omega, \mathcal{F})$, canonically built using Kolmogorov's extension theorem.

Let us try to define a specification $\gamma$ with which $\mathbb{P}$ would be consistent. Using the Markov property for random walks, the candidate is given by 
the kernel $\gamma_{\Lambda_{n}}$, defined for all cube $\Lambda_{n}=[-n, n] \cap \mathbb{Z}$ and for all $\sigma, \omega \in \Omega$ by

$$
\gamma_{\Lambda_{n}}(\sigma \mid \omega)=\mathbb{P}\left[Y_{\Lambda_{n}}=\sigma_{\Lambda_{n}} \mid Y_{-n-1}=\omega_{-n-1}, Y_{n+1}=\omega_{n+1}\right]
$$

where the event in the conditioning is the cylinder $C_{\omega_{\{n-1, n+1\}}}$, of positive $\mathbb{P}$-measure. It is straightforward to extend it to any finite $\Lambda \in \mathcal{S}$ to get a family of proper kernels $\gamma=\left(\gamma_{\Lambda}\right)_{\Lambda \in \mathcal{S}}$ that is indeed a specification. Let us assume that there exists $\mu \in \mathcal{G}(\gamma)$. Then we claim that $\mu$ cannot be a probability measure because for all $k \in \mathbb{Z}$, and for all $\epsilon>0, \mu\left[Y_{0}=k\right]<\epsilon$. The reason for this is that for all $n \in \mathbb{N}, S_{n}=Y_{n}-Y_{0}$ follows a binomial law. It is unbounded and thus for all $\epsilon>0$ and $k \in \mathbb{Z}$ and $n$ big enough, $\mathbb{P}\left[S_{n}=k\right]<\epsilon$. Using then the consistency relation $\mu \gamma_{\Lambda_{n}}=\mu$ to evaluate $\mu\left[Y_{0}=k\right]$ in terms of conditional probabilities of $\mathbb{P}$, one gets this result of "escape of mass to infinity": If $\mu \in \mathcal{G}(\gamma)$, then for all $k \in \mathbb{Z}$, and for all $\epsilon>0, \mu\left[Y_{0}=k\right] \leq \epsilon$, and thus $\mu$ cannot be a probability measure: $\mathcal{G}(\gamma)=\emptyset$.

\section{Totally random single-particle}

This example has been provided by Georgii [52]. Consider the case of a lattice gas, i.e. $E=\{0,1\}, S=\mathbb{Z}^{d}$, denote by $\mathbf{0}$ the configuration null everywhere and for any $a \in S$ consider the configuration $\sigma^{a}$ defined for all $i \in S$ by $\left(\sigma^{a}\right)_{i}=1$ iff $i=a$ (and zero otherwise), modelling the evolution of a single particle localized at the site $a$. To model a single particle evolving totally at random in a lattice gas in this DLR framework, introduce the following kernel, proper by construction:

$\forall \Lambda \in \mathcal{S}, \forall \omega \in \Omega, \forall A \in \mathcal{F}, \gamma_{\Lambda}(A \mid \omega)=\left\{\begin{array}{l}\frac{1}{|\Lambda|} \sum_{a \in \Lambda} \mathbf{1}_{A}\left(\sigma^{a}\right) \text { if } \omega_{\Lambda^{c}}=\mathbf{0}_{\Lambda^{c}} \\ \mathbf{1}_{A}\left(\mathbf{0}_{\Lambda} \omega_{\Lambda^{c}}\right)\end{array}\right.$

Firstly, one can check that the corresponding $\gamma$ is a specification and that for any sequence $\left(\mu_{n}\right)_{n}$ of probability measures on $\left(\Omega_{\Lambda_{n}}, \mathcal{F}_{\Lambda_{n}}\right)$, the sequence of probability measures $\left(\mu_{n} \gamma_{\Lambda_{n}}\right)_{n}$ converges weakly towards the Dirac measure $\delta_{\mathbf{0}}$ on the null configuration, and thus that the latter is a good candidate to be in $\mathcal{G}(\gamma)$. Nevertheless this is not the case and assuming that a measure $\mu$ belongs to $\mathcal{G}(\gamma)$, one proves [52] that

$$
\mu\left[\sum_{i \in \mathbb{Z}^{d}} \omega_{i}>1\right]=\mu\left[\sum_{i \in \mathbb{Z}^{d}} \omega_{i}=1\right]=\mu\left[\sum_{i \in \mathbb{Z}^{d}} \omega_{i}=0\right]=0
$$

using different techniques and expressions of the kernel in the three cases. Thus $\mu(\Omega)=0, \mu$ cannot be a probability measure and $\mathcal{G}(\gamma)$ is empty.

In this case, which fits in our main framework on the contrary to the first example, the non-existence comes from the dependence of the kernel 
on what happens at infinity and this cannot be controlled by the topology of weak convergence ${ }^{4}$. A good framework to ensure existence would be specifications where this influence is shielded out, and the main one corresponds to specifications that transform local functions into quasilocal ones, giving rise to the concept of quasilocal specification, central in this theory of infinite-volume Gibbs measures as we shall see next chapter.

\section{Existence and uniqueness: Reversible Markov chain}

Let us describe reversible Markov chains by means of specifications, following again a presentation of [109]. Consider $\Omega=\{-1,+1\}^{\mathbb{Z}}$ and a stochastic matrix

$$
M=\left(\begin{array}{cc}
p & 1-p \\
1-q & q
\end{array}\right)
$$

with $p>0, q>0$ such that $M$ is irreducible and aperiodic. Thus ${ }^{5}$

$$
\exists \text { unique } \nu \in \mathcal{M}_{1}^{+}(E, \mathcal{E}) \text { such that } \nu M=\nu .
$$

This defines an ergodic Markov chain $X=\left(X_{n}\right)_{n \in \mathbb{N}}$ whose conditional probabilities are given by $M$, and by Kolmogorov's existence theorem, a unique $\mathbb{P}_{\nu} \in \mathcal{M}_{1}^{+}(\Omega)$ s.t. for all $\omega \in \Omega, k, i_{1}, \cdots, i_{k} \in \mathbb{N}$,

$$
\mathbb{P}_{\nu}\left[C_{\omega_{\left\{i_{1}, \cdots, i_{k}\right\}}}\right]=\nu\left(\omega_{i_{1}}\right) \cdot M^{i_{2}-i_{1}}\left(\omega_{i_{1}}, \omega_{i_{2}}\right) \cdot \cdots \cdot M^{i_{k}-i_{k-1}}\left(\omega_{i_{k-1}}, \omega_{i_{k}}\right) .
$$

The ergodicity of the chain is crucial to get uniqueness, and we shall in particular use that

$$
\forall j, k \in E, \lim _{n \rightarrow \infty} M^{n}(j, k)=\nu(k)>0
$$

The considered Markov chain $X=\left(X_{n}\right)_{n \in \mathbb{N}}$ is then the sequence of random variables on $\left(\{-1,+1\}^{\mathbb{N}}, \mathcal{E}^{\otimes \mathbb{N}}\right)$ of law $\mathbb{P}_{\nu}$, defined on cylinders by (2.26). Writing its elementary cylinders $\left\{X_{k}(\omega)=i\right\}$ in the form $C_{\omega_{k}}=\left\{\omega_{k}=i\right\}$, one gets the Markov property: For all $\omega \in \Omega$, for all $k, i_{1}, \cdots, i_{k} \in \mathbb{N}, \forall i, j, \epsilon_{k-1}, \cdots, \epsilon_{0} \in E$

$$
\mathbb{P}_{\nu}\left[\omega_{i_{k+1}}=i \mid \omega_{i_{k}}=j, \omega_{i_{k-1}}=\epsilon_{k-1}, \cdots, \omega_{0}=\epsilon_{0}\right]=M(j, i)
$$

\footnotetext{
${ }^{4}$ We shall see later that under extra topological properties, one can construct measures in $\mathcal{G}(\gamma)$ by considering weak limits of sequences of finite-volume probability measures, with random boundary conditions.

${ }^{5}$ Our standard reference for Markov Chains is [21].
} 
This Markov chain is also reversible: $\forall k, l \in \mathbb{N}, \forall i, j, \epsilon_{k+1}, \cdots, \epsilon_{k+l} \in E$

$$
\begin{aligned}
& \mathbb{P}_{\nu}\left[\omega_{i_{k}}=i \mid \omega_{i_{k+1}}=j, \cdots, \omega_{k+l}=\epsilon_{k+l}\right] \\
= & \frac{\mathbb{P}_{\nu}\left[\omega_{i_{k}}=i, \omega_{i_{k+1}}=j, \cdots, \omega_{k+l}=\epsilon_{k+l}\right]}{\mathbb{P}_{\nu}\left[\omega_{i_{k+1}}=j, \cdots, \omega_{k+l}=\epsilon_{k+l}\right]} \\
= & \frac{\nu(i) M(i, j) \cdots M\left(\epsilon_{k+l-1}, \epsilon_{k+l}\right)}{\nu(j) M\left(j, \epsilon_{k+2}\right) \cdots M\left(\epsilon_{k+l-1}, \epsilon_{k+l}\right)} \\
= & \frac{\nu(i) M(i, j)}{\nu(j)}:=N(j, i)
\end{aligned}
$$

where $N$ is then the stochastic matrix associated to the reverse chain. Hence, we can extend this chain on $\Omega=\{-1,+1\}^{\mathbb{Z}}$, and in particular it is still ergodic. Introduce now a specification $\gamma$ such that $\mathbb{P}_{\nu} \in \mathcal{G}(\gamma)$, and compute

$$
\begin{aligned}
\mathbb{P}_{\nu}\left[\sigma_{\Lambda_{n}} \mid \sigma_{\Lambda_{n}^{c}}=\omega_{\Lambda_{n}^{c}}\right] & =\frac{\mathbb{P}_{\nu}\left[\omega_{-\infty,-n-1]} \sigma_{\Lambda_{n}} \omega_{[n+1,+\infty}\right]}{\mathbb{P}_{\nu}\left[\omega_{]-\infty,-n-1]} \omega_{[n+1,+\infty}\right]} \\
& =\frac{\mathbb{P}_{\nu}\left[\omega_{-n-1} \sigma_{\Lambda_{n}} \omega_{n+1}\right]}{\mathbb{P}_{\nu}\left[\omega_{-n-1} \omega_{n+1}\right]} \\
& =\frac{\nu\left(\omega_{-n-1}\right) M\left(\omega_{-n-1}, \sigma_{-n}\right) \cdots M\left(\sigma_{n}, \omega_{n+1}\right)}{\nu\left(\omega_{-n-1}\right) M^{2 n+2}\left(\omega_{-n-1}, \omega_{n+1}\right)} \\
& =\frac{M\left(\omega_{-n-1}, \sigma_{-n}\right) \cdots M\left(\sigma_{n}, \omega_{n+1}\right)}{M^{2 n+2}\left(\omega_{-n-1}, \omega_{n+1}\right)} .
\end{aligned}
$$

Denote then the (finite) normalization by $\mathbf{Z}_{\Lambda_{n}}(\omega)=M^{2 n+2}\left(\omega_{-n-1}, \omega_{n+1}\right)$ and define a proper kernel $\gamma_{\Lambda_{n}}$ on $(\Omega, \mathcal{F})$, for all $\sigma \in \Omega$, by

$$
\gamma_{\Lambda_{n}}(\sigma \mid \omega)=\frac{1}{\mathbf{Z}_{\Lambda_{n}}(\omega)} \cdot M\left(\omega_{-n-1}, \sigma_{-n}\right) \cdots M\left(\sigma_{n}, \omega_{n+1}\right) .
$$

One can check that we define thus a specification $\gamma$ such that $\mathbb{P}_{\nu} \in \mathcal{G}(\gamma)$ : In contrast to the modelization of the simple random walk described above, the existence of a DLR measure is then insured. Let us consider now any $\mu \in \mathcal{G}(\gamma)$ and prove that $\mu=\mathbb{P}_{\nu}$. To do so, it is enough to prove for all $\omega \in \Omega$, for all $k, i_{1}, \cdots, i_{k} \in \mathbb{N}$,

$$
\mathbb{P}_{\nu}\left[C_{\omega_{\left\{i_{1}, \cdots, i_{k}\right\}}}\right]=\mu\left[\left(\omega_{i_{1}}, \cdots, \omega_{i_{k}}\right)\right] .
$$

Let us prove it for the one-dimensional cylinder using Markov property and consistency: We have for all $x \in \mathbb{E}$ and $n \in \mathbb{N}$

$$
\begin{aligned}
\mu\left[\sigma_{0}=x\right] & =\sum_{i, j \in E} \mu\left[\sigma_{0}=x \mid \sigma_{-n-1}=i, \sigma_{n+1}=j\right] \mu\left[\sigma_{-n-1}=i, \sigma_{n+1}=j\right] \\
& =\sum_{i, j \in E} \frac{M^{n+1}(i, x) \cdot M^{n+1}(x, j)}{M^{2 n+2}(i, j)} \cdot \mu\left[\sigma_{-n-1}=i, \sigma_{n+1}=j\right] .
\end{aligned}
$$


Taking now the limit when $n$ goes to infinity, one gets

$$
\begin{aligned}
\mu\left[\sigma_{0}=x\right] & =\sum_{i, j \in E} \frac{\nu(x) \cdot \nu(j)}{\nu(j)} \cdot \mu\left[\sigma_{-n-1}=i, \sigma_{n+1}=j\right] \\
& =\nu(x) \sum_{i, j \in E} \mu\left[\sigma_{-n-1}=i, \sigma_{n+1}=j\right] \\
& =\nu(x)=\mathbb{P}_{\nu}\left[\sigma_{0}=x\right] .
\end{aligned}
$$

We obtain the equality of these measures on the other cylinders in the same way. Thus $\mathcal{G}(\gamma)$ is the singleton ${ }^{6}\left\{\mathbb{P}_{\nu}\right\}$.

\section{Example of phase transition: Ferromagnetic 2d-Ising model}

The Ising model is the original archetype Gibbs specification and we present it briefly at dimension $d=2$, temperature $T=\frac{1}{\beta}>0$ and no external field, as originally introduced by Lenz to model ferromagnetism ${ }^{7}$. To do so, one considers microscopic magnets $\sigma_{i} \in E=\{-1,+1\}$ at each site $i \in \mathbb{Z}^{2}$, and to express the fact that neighbors have a tendency to align, the following nearest neighbor potential. It is a family $\Phi=\left(\Phi_{A}\right)_{A \in \mathcal{S}}$ of $\mathcal{F}_{A}$-measurable functions defined for all $\omega \in \Omega$ by

$$
\Phi_{A}(\omega)=\left\{\begin{array}{l}
-J \omega_{i} \omega_{j} \text { if } A=\langle i j\rangle \\
0 \quad \text { otherwise. }
\end{array}\right.
$$

The corresponding Hamiltonian at finite volume $\Lambda \in \mathcal{S}$, temperature $\beta^{-1}>0$, coupling $J>0$ and boundary condition $\omega \in \Omega$ is the welldefined $^{8}$ function on $\Omega \times \Omega$ defined by

$$
H_{\Lambda}^{\beta \Phi}(\sigma \mid \omega)=\sum_{A \in \mathcal{S}, A \cap \Lambda \neq \emptyset} \Phi_{A}\left(\sigma_{\Lambda} \omega_{\Lambda^{c}}\right) .
$$

The Gibbs specification at inverse temperature $\beta>0$ is the family of probability kernels $\gamma^{\beta \Phi}=\left(\gamma_{\Lambda}^{\beta \Phi}\right)_{\Lambda \in \mathcal{S}}$ given for all $\Lambda \in \mathcal{S}, \sigma, \omega \in \Omega$ by

$$
\gamma_{\Lambda}^{\beta \Phi}(\sigma \mid \omega)=\frac{1}{Z_{\Lambda}^{\beta \Phi}(\omega)} e^{-\beta H_{\Lambda}(\sigma \mid \omega)},
$$

\footnotetext{
${ }^{6}$ Remark that the terms involving the measure $\nu$ cancel out in the specification. Nevertheless, its invariant character is encoded in the conditioning, yielding a DLR measure that depends on $\nu$.

${ }^{7}$ Ising analyzed this model in one dimension in its thesis supervised by Lenz in 1922 [71]. The only contribution of that work to higher dimensions was the wrong interpretation that just as in $d=1$, in higher dimension there is no phase transition.

${ }^{8}$ It is a finite sum here but this is not the case in general. One has usually to check summability conditions on the potential to define Gibbs measures, as we shall see in the next section.
} 
where the partition function $Z_{\Lambda}^{\beta \Phi}(\omega)$ is a standard normalization depending on the boundary condition $\omega$. It is indeed a specification due to the expression of the Hamiltonian in terms of a sum over local potential terms (see [52] or in next section). Intensive studies have established the following:

Theorem 2.27 (Phase transition at low $\mathbf{T}$ ). There exists $\beta_{c}>0$ s.t.

- There exists a unique measure consistent with $\gamma^{\beta \Phi}$ at high temperatures $\beta<\beta_{c}$.

- At low temperatures $\beta>\beta_{c}$, the set $\mathcal{G}\left(\gamma^{\beta \Phi}\right)$ is the Choquet simplex $\left[\mu_{\beta}^{-}, \mu_{\beta}^{+}\right]$whose extremal elements are mutually singular and can be selected by the weak limits

$$
\mu_{\beta}^{ \pm}(\cdot)=\lim _{\Lambda \uparrow \mathcal{S}} \gamma_{\Lambda}(\cdot \mid \pm)
$$

with the magnetizations satisfying

$$
\mu_{\beta}^{+}\left[\sigma_{0}\right]=-\mu_{\beta}^{-}\left[\sigma_{0}\right]=M_{0}>0 .
$$

The existence of the weak limits is usually proved here using correlation or related (GKS, FKG, etc.) inequalities valid for some ferromagnetic systems. The existence of a critical temperature has been qualitatively established by Peierls in 1936 [103, 55], using a geometrical computation based on the energy of contours, that are circuits in the dual of the lattice associated to a configuration, whose lengths are related to its energy. His analysis gave rise to the powerful Pirogov-Sinai theory of phase transitions for more general models $[106,36]$. The exact value of $\beta_{c}$ is due to Kramers and Wannier in 1941 [70], while Yang obtained the magnetization in 1951 [121], both using algebraic tools ${ }^{9}$. The full convex picture, restricted to translation-invariant measures, has been independently proved by Aizenmann [1] and Higuchi [58], both inspired by considerations on percolation raised by Russo [111], described in [53]. This picture has been recently extended to higher dimension by Bodineau [9]. The fact that $\mathcal{G}\left(\gamma^{\beta \Phi}\right)$ is the Choquet simplex $\left[\mu_{\beta}^{-}, \mu_{\beta}^{+}\right]$means that any measure $\mu \in \mathcal{G}\left(\gamma^{\beta \Phi}\right)$ is uniquely determined by a convex combination of the extreme phases $\mu_{\beta}^{ \pm}$, i.e. that there exists a unique $\alpha \in[0,1]$ s.t. $\mu=\alpha \mu_{\beta}^{-}+(1-\alpha) \mu_{\beta}^{+}$. The situation is more complex in higher dimension or on other lattices, as we shall see.

\subsection{Convexity theory of DLR-measures}

In this subsection, before introducing Gibbs measures properly speaking within the nice topological framework of quasilocality, we enlarge our finite

\footnotetext{
${ }^{9}$ The magnetization has been conjectured in an unpublished manuscript by Onsager in 1949, who also rigorously derived the free energy in 1944 [102].
} 
single-spin state-space settings and first study the general structure of the set $\mathcal{G}(\gamma)$ of probability measures consistent with a specification $\gamma$ on a general Polish probability space $(\Omega, \mathcal{F})$, product of ${ }^{10}$ a finite single-spin state-space $(E, \mathcal{E})$. In this case, the family $\mathcal{C}$ of cylinders has the nice following property of being a countable core, following a terminology of [52]:

Definition 2.28 (Countable core). A countable family $\mathcal{C} \subset \mathcal{F}$ is said to be a countable core if it has the following properties:

1. $\mathcal{C}$ generates $\mathcal{F}$ and is stable under finite intersections ${ }^{11}$.

2. If $\left(\mu_{n}\right)_{n \in \mathbb{N}}$ is a sequence of $\mathcal{M}_{1}^{+}(\Omega)$ such that $\lim _{n \rightarrow \infty} \mu_{n}(C)$ exists for any cylinder $C \in \mathcal{C}$, then there exists a unique $\mu \in \mathcal{M}_{1}^{+}(\Omega)$ that coincides with this limit on $\mathcal{C}$ :

$$
\forall C \in \mathcal{C}, \mu(C)=\lim _{n \rightarrow \infty} \mu_{n}(C) .
$$

The proof that the family of cylinders $\mathcal{C}$ is indeed a core relies on Carathéodory's extension theorem $[52,118]$. We exploit this property to define some weights $\alpha_{\mu}(M)$ associated with any measurable subset of measures $M \in e(\operatorname{ex} \mathcal{G}(\gamma))$ by

$$
\alpha_{\mu}(M)=\mu\left[\left\{\omega \in \Omega: \exists \nu \in M, \lim _{n} \gamma_{\Lambda_{n}}(C \mid \omega)=\nu(C), \forall C \in \mathcal{C}\right\}\right] .
$$

The main purpose of this section is to use this property to provide a general description of $\mathcal{G}(\gamma)$ when it is not an empty set. Conditions ensuring existence are described in Chapter 3.

\subsubsection{Choquet simplex of DLR-measures}

Theorem 2.31. Assume that $\mathcal{G}(\gamma) \neq \emptyset$. Then $\mathcal{G}(\gamma)$ is a convex subset of $\mathcal{M}_{1}^{+}(\Omega, \mathcal{F})$ whose extreme boundary is denoted ex $\mathcal{G}(\gamma)$, and satisfies the following properties:

1. The extreme elements of $\mathcal{G}(\gamma)$ are the probability measures $\mu \in \mathcal{G}(\gamma)$ that are trivial on the tail $\sigma$-field $\mathcal{F}_{\infty}$ :

$$
\operatorname{ex} \mathcal{G}(\gamma)=\left\{\mu \in \mathcal{G}(\gamma): \mu(B)=0 \text { or } 1, \forall B \in \mathcal{F}_{\infty}\right\}
$$

Moreover, distinct extreme elements $\mu, \nu \in \operatorname{ex} \mathcal{G}(\gamma)$ are mutually singular: $\exists B \in \mathcal{F}_{\infty}, \mu(B)=1$ and $\nu(B)=0$, and more generally, each $\mu \in \mathcal{G}(\gamma)$ is uniquely determined within $\mathcal{G}(\gamma)$ by its restriction to $\mathcal{F}_{\infty}$.

\footnotetext{
${ }^{10}$ In a more general set-up, $(E, \mathcal{F})$ has to be a standard Borel space, see [52].

${ }^{11}$ This property corresponds to what is called a $\pi$-system, see in the next section.
} 
2. $\mathcal{G}(\gamma)$ is a Choquet simplex: Any $\mu \in \mathcal{G}(\gamma)$ can be written in a unique way as

$$
\mu=\int_{\operatorname{ex\mathcal {G}(\gamma )}} \nu \cdot \alpha_{\mu}(d \nu),
$$

where $\alpha_{\mu} \in \mathcal{M}_{1}^{+}(\operatorname{ex} \mathcal{G}(\gamma), e(\operatorname{ex} \mathcal{G}(\gamma)))$ is defined by (2.30) for all $M \in$ $e(\operatorname{ex} \mathcal{G}(\gamma))$.

In particular, when $M$ is a singleton $\{\nu\} \in e(\operatorname{ex} \mathcal{G}(\gamma))$, (2.30) reads

$$
\alpha_{\mu}(\{\nu\})=\mu\left[\left\{\omega \in \Omega: \lim _{n} \gamma_{\Lambda_{n}}(\cdot \mid \omega)=\nu(\cdot)\right\}\right] .
$$

The convexity of $\mathcal{G}(\gamma)$ is trivial and 1 . will be a direct consequence of the following lemma, also crucial to prove 2. To state this lemma, we recall the absolute continuity of measures: We say $\nu \in \mathcal{M}_{1}^{+}(\Omega)$ is absolutely continuous with respect to $\mu$, and we write it $\nu<<\mu$, if and only if

$$
\forall A \in \mathcal{F}, \mu(A)=0 \Longrightarrow \nu(A)=0 .
$$

We also recall that $\mu \in \mathcal{G}(\gamma)$ is extreme iff

$$
\mu=\alpha \nu+(1-\alpha) \bar{\nu} \text { with } \alpha \in] 0,1[\text { and } \nu, \bar{\nu} \in \mathcal{G}(\gamma) \Longrightarrow \nu=\bar{\nu}=\mu \text {. }
$$

Lemma 2.35. Assume that $\mu \in \mathcal{G}(\gamma)$ is such that

$$
\mu=\alpha \nu+(1-\alpha) \bar{\nu}, \text { with } \alpha \in] 0,1\left[, \nu, \bar{\nu} \in \mathcal{M}_{1}^{+}(\Omega) .\right.
$$

Then $\nu<<\mu, \bar{\nu}<<\mu$ and

$$
\nu \in \mathcal{G}(\gamma) \Longleftrightarrow f:=\frac{d \nu}{d \mu} \in \mathcal{F}_{\infty}
$$

Proof: Let $\mu, \nu, \bar{\nu}$ and $\alpha$ as above. The absolute continuity of $\nu$ w.r.t. $\mu$ comes trivially from the positiveness of probability measures: Take $A \in \mathcal{F}$ with $\mu(A)=0$, then $\alpha \nu(A)+(1-\alpha) \bar{\nu}(A)=0$ implies $\nu(A)=\bar{\nu}(A)=0$ because $0<\alpha<1$, and thus $\nu<<\mu$ and $\bar{\nu}<<\mu$. Now let us prove (2.36). We follow ${ }^{12}$ mostly the proof of [52] and introduce first two $\sigma$-algebras related to the specification $\gamma$ : The $\sigma$-algebra of $\gamma$-invariant measurable sets

$$
\mathcal{F}_{\gamma}=\left\{A \in \mathcal{F}: \gamma_{\Lambda}(A \mid \cdot)=\mathbf{1}_{A}(\cdot), \forall \Lambda \in \mathcal{S}\right\}
$$

and $^{13}$ the $\sigma$-algebra of $\mu$-almost surely $\gamma$-invariant measurable sets

$$
\mathcal{F}_{\gamma}(\mu)=\left\{A \in \mathcal{F}: \gamma_{\Lambda}(A \mid \cdot)=\mathbf{1}_{A}(\cdot) \mu \text {-a.s., } \forall \Lambda \in \mathcal{S}\right\} .
$$

\footnotetext{
${ }^{12}$ More generally, the proof comes from [31], but we have rewritten it to avoid the introduction of too many concepts. These ideas are also related to the so-called disintegration of measures, see $[3,4,101]$.

${ }^{13}$ They are indeed $\sigma$-algebras [52].
} 
We first prove that for a given $\mu \in \mathcal{G}(\gamma)$,

$$
\nu \in \mathcal{G}(\gamma) \Longleftrightarrow f:=\frac{d \nu}{d \mu} \in \mathcal{F}_{\gamma}(\mu)
$$

To prove the implication $\Rightarrow$, it is enough to prove that for all $c \in[0,1]$ the event $\{f \geq c\} \in \mathcal{F}_{\gamma}(\mu)$. For $\Lambda \in \mathcal{S}$, we want to prove that, whenever $\mu, \nu \in \mathcal{G}(\gamma)$ and $f=\frac{d \nu}{d \mu}$,

$$
\gamma_{\Lambda}(f \geq c \mid \cdot)=\mathbf{1}_{f \geq c}(\cdot), \quad \mu-a . s .
$$

or equivalently that when $g=\mathbf{1}_{f \geq c}$, one has

$$
\gamma_{\Lambda} g=g, \quad \mu-\text { a.s. }
$$

Now, $\mu \in \mathcal{G}(\gamma)$ implies $\mu\left[\gamma_{\Lambda} g-g\right]=0$, so to prove $(2.38)$ it is enough to prove

$$
\gamma_{\Lambda} g \leq g, \quad \mu-\text { a.s. }
$$

Writing $\gamma_{\Lambda} g=\left(\gamma_{\Lambda} g\right) \cdot \mathbf{1}_{f \geq c}+\left(\gamma_{\Lambda} g\right) \cdot \mathbf{1}_{f<c}=g \cdot \gamma_{\Lambda} g+\mathbf{1}_{f<c} \cdot \gamma_{\Lambda} g$, one gets that proving

$$
\left(\gamma_{\Lambda} g\right) \cdot \mathbf{1}_{f<c}=0, \quad \mu-\text { a.s. }
$$

will be enough to get (2.39) and thus (2.38). To do so, let us prove that

$$
\int_{\{f<c\}}(f-c) \cdot \gamma_{\Lambda} g d \mu \geq 0
$$

i.e. that

$$
\int_{\{f<c\}} f \cdot \gamma_{\Lambda} g d \mu \geq c \cdot \int_{\{f<c\}} \gamma_{\Lambda} g d \mu .
$$

Using $\nu=f \mu, \nu \gamma_{\Lambda}=\nu, \mu=\mu \gamma_{\Lambda}$ and the expression for $g$, one writes

$$
\begin{aligned}
\int_{f<c} f \cdot \gamma_{\Lambda} g d \mu & =\int_{\Omega} f \cdot \gamma_{\Lambda} g d \mu-\int_{f \geq c} f \cdot \gamma_{\Lambda} g d \mu \\
& =\int_{\Omega} f \cdot \gamma_{\Lambda} g d \mu-\int_{\Omega} f \cdot g \cdot \gamma_{\Lambda} g d \mu \\
& =\int_{\Omega} \gamma_{\Lambda} g d \nu-\int_{\Omega} f \cdot g \cdot \gamma_{\Lambda} g d \mu \\
& =\int_{\Omega} g d \nu-\int_{\Omega} f \cdot g \cdot \gamma_{\Lambda} g d \mu \\
& =\int_{\Omega} f \cdot g d \mu-\int_{\Omega} f \cdot g \gamma_{\Lambda} g d \mu \\
& =\int_{\Omega} f \cdot g \cdot\left(1-\gamma_{\Lambda} g\right) d \mu .
\end{aligned}
$$


But $f \cdot g=f \cdot \mathbf{1}_{f \geq c} \geq c \cdot \mathbf{1}_{f \geq c}=c \cdot g$, and because $0 \leq \gamma_{\Lambda} g \leq 1$, one gets

$\int_{f<c} f \cdot \gamma_{\Lambda} g d \mu \geq c \cdot \int_{\Omega} g \cdot\left(1-\gamma_{\Lambda} g\right) d \mu=c \cdot \mu[g]-c \cdot \mu\left[g \gamma_{\Lambda} g\right]=c \cdot \int_{f<c} \gamma_{\Lambda} g d \mu$

where the last equality has been obtained using the consistency relation $\mu[g]=\mu\left[\gamma_{\Lambda} g\right]$. So (2.42) holds, which in turns implies (2.41) and then (2.40) because trivially $(f-c)$ is strictly negative on the event $\{f<c\}$, implying thus $\gamma_{\Lambda} g=0$ on the same event, that is exactly (2.39). Thus one has $\gamma_{\Lambda} g=g \quad \mu$-a.s., and eventually that the density $f \in \mathcal{F}_{\gamma}(\mu)$.

Let us now prove that for $\mu \in \mathcal{G}(\gamma)$ and $f=\frac{d \nu}{d \mu}$,

$$
f \in \mathcal{F}_{\gamma}(\mu) \Longrightarrow \nu=f \cdot \mu \in \mathcal{G}(\gamma)
$$

It is enough to prove it for a step function $f=\mathbf{1}_{A}$, with $A \in \mathcal{F}_{\gamma}(\mu)$, so let us prove that for all $\Lambda \in \mathcal{S}$ and $A \in \mathcal{F}_{\gamma}(\mu)$, the measure

$$
\nu(\cdot):=\mathbf{1}_{A}(\cdot) \mu(\cdot)=\mu(\cdot \cap A)
$$

satisfies $\nu \gamma_{\Lambda}=\nu$. By the defining properties of a specification, one can write, for all $B \in \mathcal{F}$ and $\Lambda \in \mathcal{S}$,

$$
\begin{aligned}
\nu \gamma_{\Lambda}(B)=\int_{\Omega} \gamma_{\Lambda}(B \mid \cdot) d \nu & =\nu\left[\gamma_{\Lambda}(A \cap B \mid \cdot)\right]+\nu\left[\gamma_{\Lambda}(B \backslash A \mid \cdot)\right] \\
& \leq \mu\left[\gamma_{\Lambda}(A \cap B \mid \cdot)\right]+\mu\left[\mathbf{1}_{A}(\cdot) \gamma_{\Lambda}(\Omega \backslash A \mid \cdot)\right] \\
& =\mu(A \cap B)+\mu\left[\mathbf{1}_{A}(\cdot) \mathbf{1}_{\Omega \backslash A}(\cdot)\right]=\nu(B) .
\end{aligned}
$$

Working similarly on $B^{c}$ one also gets the domination of $\nu \gamma_{\Lambda}\left(B^{c}\right)$ by $\nu\left(B^{c}\right)$, and together with

$$
\nu \gamma_{\Lambda}(B)+\nu \gamma_{\Lambda}\left(B^{c}\right)=1=\nu(B)+\nu\left(B^{c}\right)
$$

this implies $\nu \gamma_{\Lambda}(B)=\nu(B), \forall B \in \mathcal{F}$ and $\Lambda \in \mathcal{S}$, so $\nu \in \mathcal{G}(\gamma)$.

To conclude, realize first that $\mathcal{F}_{\infty}$ is exactly the $\gamma$-invariant $\sigma$-algebra $\mathcal{F}_{\gamma}$ : Any $A \in \mathcal{F}_{\infty}$ is $\gamma$-invariant by properness, and reciprocally, any $\gamma$ invariant set $A \in \mathcal{F}_{\infty}$, because it can be written $A=\left\{\gamma_{\Lambda}(A \mid \cdot)=1\right\}$ for any $\Lambda \in \mathcal{S}$. Eventually, tail-triviality is obtained because $\mu$ is trivial on $\mathcal{F}_{\gamma}(\mu)$ if and only if it is trivial on $\mathcal{F}_{\gamma}=\mathcal{F}_{\infty}$, the $\mu$-completion of the latter being exactly ${ }^{14} \mathcal{F}_{\gamma}(\mu)$, see [52].

\footnotetext{
${ }^{14} \mathrm{It}$ is not the case for general kernels, properness of the specification is crucial [52].
} 


\section{Proof of Theorem 2.31:}

1. It is straightforward to check that $\mathcal{G}(\gamma)$ is a convex subset of $\mathcal{M}_{1}^{+}(\Omega)$. Then $\operatorname{ex} \mathcal{G}(\gamma)$ is non-empty ${ }^{15}$ and suppose $\mu$ is one of its extreme elements, and that there exists $B \in \mathcal{F}_{\infty}$ with $0<\mu(B)<1$. Then the conditional probabilities w.r.t. $B$ and its complement $B^{c}$ are well-defined as probability measures on $(\Omega, \mathcal{F})$ in such a way that

$$
\mu(\cdot)=\mu(\cdot \mid B) \mu(B)+\mu\left(\cdot \mid B^{c}\right) \mu\left(B^{c}\right)
$$

or equivalently

$$
\mu(\cdot)=\alpha \mu(\cdot \mid B)+(1-\alpha) \mu\left(\cdot \mid B^{c}\right)
$$

with $\alpha=\mu(B) \in] 0,1[$. Denote $\nu(\cdot)=\mu(\cdot \mid B)$ and rewrite

$$
\nu(\cdot)=\frac{\mu(\cdot \cap B)}{\mu(B)}=\frac{\mathbf{1}_{B}(\cdot)}{\mu(B)} \cdot \mu(\cdot)
$$

in such a way that $\nu<<\mu$, with a density $\frac{d \nu}{d \mu}=\frac{\mathbf{1}_{B}(\cdot)}{\mu(B)}$ that belongs to $\mathcal{F}_{\infty}$ because $B$ is a tail event. Lemma 2.35 implies thus that $\nu(\cdot)=\mu(\cdot \mid B)$ and $\bar{\nu}(\cdot)=\mu\left(\cdot \mid B^{c}\right)$ are distinct elements of $\mathcal{G}(\gamma)$, and together with (2.43) and $0<\alpha<1$, this contradicts the extremality of $\mu$. Thus, such a tail event $B \in \mathcal{F}_{\infty}$ cannot exist and one gets the first part of item 1 . of this theorem:

$$
\mu \in \operatorname{ex} \mathcal{G}(\gamma) \Longrightarrow \mu(B)=0 \text { or } 1, \forall B \in \mathcal{F}_{\infty}
$$

To prove the converse statement, consider $\mu \in \mathcal{G}(\gamma)$, trivial on $\mathcal{F}_{\infty}$ and such that there exists $\alpha \in] 0,1[$ and $\nu, \bar{\nu} \in \operatorname{ex} \mathcal{G}(\gamma)$ with $\mu=\alpha \nu+(1-\alpha) \bar{\nu}$. Then by Lemma $2.35, \nu<<\mu$ with a density $f:=\frac{d \nu}{d \mu} \in \mathcal{F}_{\infty}$. The latter is a density thus $\mu[f]=1$ and by tail-triviality of $\mu$ one also has $\mu[f]=f$ ( $\mu$-a.s.). Hence $f=1$ ( $\mu$-a.s.) and $\nu=\bar{\nu}=\mu$, which is thus an extreme element of $\mathcal{G}(\gamma)$ :

$$
\mu(B)=0 \text { or } 1, \forall B \in \mathcal{F}_{\infty} \Longrightarrow \mu \in \operatorname{ex} \mathcal{G}(\gamma)
$$

Let us prove now that any $\mu \in \mathcal{G}(\gamma)$ is uniquely determined within $\mathcal{G}(\gamma)$ by its restriction to $\mathcal{F}_{\infty}$. Consider $\mu, \nu \in \mathcal{G}(\gamma)$ such that $\mu(B)=\nu(B)$ for all $B \in \mathcal{F}_{\infty}$ and the combination $\bar{\mu}=\frac{1}{2} \mu+\frac{1}{2} \nu$, which is also in $\mathcal{G}(\gamma)$ by convexity . By Lemma 2.35 one can write $\mu=f \cdot \bar{\mu}$ and $\nu=g \cdot \bar{\mu}$ with $f, g \in \mathcal{F}_{\infty}$. If $\nu=\mu$ on $\mathcal{F}_{\infty}$, then one also has $\bar{\mu}=\nu=\mu$ on $\mathcal{F}_{\infty}$, thus $f=g=1$ and $\nu=\mu$. In particular, distinct extreme elements are mutually singular because they are trivial on the tail- $\sigma$-algebra: There exists then $B \in \mathcal{F}_{\infty}$ such that $\mu(B)=1$ and $\nu(B)=0$.

${ }^{15}$ Consider for example a regular version of $\mu\left[\cdot \mid \mathcal{F}_{\infty}\right]$ for $\mu \in \mathcal{G}(\gamma)$. 
Hence, we have now a characterization of extremality in terms of tailtriviality. To see how this leads to a unique simplicial decomposition, we shall also use the following characterization of extremal DLR measures, which can be derived from (2.32) using standard arguments:

$$
\operatorname{ex} \mathcal{G}(\gamma)=\left\{\mu \in \mathcal{G}(\gamma): \mu\left[A \mid \mathcal{F}_{\infty}\right]=\mu(A), \mu \text {-a.s., } \forall A \in \mathcal{F}\right\} .
$$

2. To get the unique extreme decomposition of $\mu \in \mathcal{G}(\gamma)$, we follow the spirit of the proof of Dynkin [31] as worked out in detail by Georgii [52] in our particular DLR case. We shall mention at the end of this chapter other frameworks where such a decomposition holds.

We start thus from a specification $\gamma$ for which there exists $\mu \in \mathcal{G}(\gamma)$. By consistency, for any $n \in \mathbb{N}$, the kernel $\gamma_{\Lambda_{n}}$ is a regular version of conditional probability of $\mu$ given $\mathcal{F}_{\Lambda_{n}^{c}}$ :

$$
\gamma_{\Lambda_{n}}(A \mid \cdot)=\mu\left[A \mid \mathcal{F}_{\Lambda_{n}^{c}}\right](\cdot), \mu-\text { a.s., } \forall A \in \mathcal{F} .
$$

The existence of such a regular version of conditional probabilities is ensured by Theorem 2.13, and we shall sometimes denote formally by $\Omega_{\mu}$ the set of full $\mu$-measure set on which (2.45) holds for all $A$ and for all $n$. Remark that the uniformity in $n$ implies that $\Omega_{\mu}$ is a tail-event.

The backward martingale theorem [118] ensures then that the following almost-sure limit

$$
\lim _{n \rightarrow \infty} \mu\left[C \mid \mathcal{F}_{\Lambda_{n}^{c}}\right](\cdot)=\mu\left[C \mid \mathcal{F}_{\infty}\right](\cdot), \forall C \in \mathcal{C}
$$

exists also on a full measure set (that can be assumed to be the same $\Omega_{\mu}$ ), defining a regular version of the conditional probability w.r.t. the tail $\sigma$ algebra $\mathcal{F}_{\infty}$. The regularity of such versions of conditional probabilities is encoded in the order of the locutions " $\mu$-a.-s." and " $\forall A \in \mathcal{F}$ ".

Our strategy is now to combine (2.45) and (2.46) with the core property (Definition 2.28) to introduce appropriate objects for a decomposition of DLR measures when applied on the countable family of cylinders first, to derive some (tail-) measurability properties using countability, and thereafter to extend in a standard way the latter objects onto probability kernels that are in some sense extreme " $\mu$-almost surely". In fact, the starting point of the decomposition is one of the defining properties of versions of conditional probability with respect to the tail $\sigma$-algebra:

$$
\forall \mu \in \mathcal{M}_{1}^{+}(\Omega), \mu(\cdot)=\int_{\Omega} \mu\left[\cdot \mid \mathcal{F}_{\infty}\right](\omega) d \mu(\omega) .
$$

In some informal sense, the regular versions of $\mu\left[\cdot \mid \mathcal{F}_{\infty}\right]$ are the prototypes of extremal measures entering in the decomposition of any $\mu \in \mathcal{G}(\gamma)$. 
To formalize this using consistency and the backward martingale theorem, we need to carefully define appropriate asymptotics of the specification $\gamma$, that will be probability kernels with an asymptotic properness leading to tail triviality, that eventually leads to a concentration on the extreme elements of $\mathcal{G}(\gamma)$.

\section{Step 1: $\mu$-asymptotics of the specification}

We use first the core property (2.29) to " $\mu$-almost-surely" extend our asymptotic kernels from a definition on the cylinders. Indeed, combining (2.45) and (2.46), one gets that

$$
\forall \omega \in \Omega_{\mu}, \lim _{n \rightarrow \infty} \gamma_{\Lambda_{n}}(C \mid \omega)=\mu\left[C \mid \mathcal{F}_{\infty}\right](\omega), \forall C \in \mathcal{C}
$$

By the core property, there exists then, for each $\omega \in \Omega_{\mu}$, a measure $\pi^{\omega} \in$ $\mathcal{M}_{1}^{+}(\Omega)$ s.t.

$$
\forall C \in \mathcal{C}, \pi^{\omega}(C)=\lim _{n \rightarrow \infty} \gamma_{\Lambda_{n}}(C \mid \omega)=\mu\left[C \mid \mathcal{F}_{\infty}\right](\omega)
$$

Extending this construction to any $\omega \in \Omega_{\mu}^{c}$ by requiring $\pi^{\omega}$ to be any arbitrary elements of $\mathcal{M}_{1}^{+}(\Omega)$, one gets a probability kernel with nice specific properties:

Lemma 2.49. Let $\mu_{0} \in \mathcal{M}_{1}^{+}(\Omega), \mu \in \mathcal{G}(\gamma), \Omega_{\mu} \in \mathcal{F}_{\infty}$ as above for which (2.45) and (2.46) hold, and define $\pi^{*}: \mathcal{F} \times \Omega \longrightarrow[0,1] ;(A, \omega) \longmapsto \pi^{\omega}(A)$ as follows:

- $\forall \omega \in \Omega_{\mu}, \pi^{\omega}$ is the unique element of $\mathcal{M}_{1}^{+}(\Omega)$ such that

$$
\forall C \in \mathcal{C}, \pi^{\omega}(C)=\lim _{n} \gamma_{\Lambda_{n}}(C \mid \omega)=\mu\left[C \mid \mathcal{F}_{\infty}\right](\omega)
$$

- $\forall \omega \in \Omega_{\mu}^{c}, \pi^{\omega}$ is chosen to be the arbitrary probability measure $\mu_{0}$.

Then $\pi$ is a probability kernel from $\left(\Omega, \mathcal{F}_{\infty}\right)$ to $(\Omega, \mathcal{F})$ such that

$$
\mu \text {-a.s., } \mu\left[A \mid \mathcal{F}_{\infty}\right]=\pi^{\cdot}(A), \forall A \in \mathcal{F} \text {. }
$$

2.

$$
\left\{\pi^{\cdot} \in \mathcal{G}(\gamma)\right\}:=\left\{\omega \in \Omega: \pi^{\omega} \in \mathcal{G}(\gamma)\right\} \in \mathcal{F}_{\infty}
$$

and $\pi \cdot$ is $\mu$-a.s. consistent with $\gamma$ in the sense that:

$$
\mu\left[\pi^{\cdot} \in \mathcal{G}(\gamma)\right]=1
$$


Proof: To prove that $\pi$ is a probability kernel from $\left(\Omega, \mathcal{F}_{\infty}\right)$ to $(\Omega, \mathcal{F})$, we need to prove (see Definition 2.15) that for all $\omega \in \Omega, \pi^{\omega}(\cdot)$ is a probability measure on $(\Omega, \mathcal{F})$, and that for all $A \in \mathcal{F}, \pi^{\cdot}(A)$ is $\mathcal{F}_{\infty}$-measurable. The first item is true by construction, thanks to the core property. To prove the second one, denote $\mathcal{D}=\left\{A \in \mathcal{F}: \pi^{\cdot}(A) \in \mathcal{F}_{\infty}\right\}$. By construction, $\mathcal{D}$ contains the set $\mathcal{C}$ of cylinders. The latter is a $\pi$-system (i.e. a family of sets stable by finite intersections, that generates the $\sigma$-algebra $\mathcal{F}$ ) whereas $\mathcal{D}$ is a Dynkin system (i.e. a family of subsets of $\Omega$ that contains $\Omega$ and is stable for subtractions of subsets and under monotone limits of sets) contained in $\mathcal{F}$. Then we use

Lemma 2.52 (Dynkin lemma [118]). Any Dynkin system which contains a $\pi$-system contains the $\sigma$-algebra generated by this $\pi$-system.

Thus the property characterizing $\mathcal{D}$ extends to the whole $\sigma$-algebra $\mathcal{F}$, because the former is generated by the $\pi$-system of the cylinder, and thus: $\forall A \in \mathcal{F}, \pi^{\cdot}(A) \in \mathcal{F}_{\infty}$. By construction, the equality $\pi^{\omega}(C)=\mu\left[C \mid \mathcal{F}_{\infty}\right]$ is true for all cylinders $C$ for $\mu$-almost every $\omega$ by (2.48). For these $\omega$ 's, $\mu\left[\cdot \mid \mathcal{F}_{\infty}\right](\omega)$ and $\pi^{\omega}$ are two probability measures that coincide on a $\pi$ system, which coincide then on the $\sigma$-algebra generated by this $\pi$-system, which is $\mathcal{F}$ itself here. This proves item 1 . of the lemma.

Fix now a cylinder $C \in \mathcal{C}$ and focus first on the (random) measures $\pi^{\cdot}(C)$. By construction, they inherit the properness property and in particular for $\mu$-almost every $\omega \in \Omega$,

$$
\forall C \in \mathcal{C}_{\infty}, \quad \pi^{\omega}(C)=\mathbf{1}_{C}(\omega)
$$

Also inherited from consistency: For $\mu$-almost every $\omega$,

$$
\forall C \in \mathcal{C}, \pi^{\omega} \gamma_{\Lambda}(C)=\mu\left[\mu\left[C \mid \mathcal{F}_{\Lambda^{c}}\right] \mid \mathcal{F}_{\infty}\right]=\mu\left[C \mid \mathcal{F}_{\infty}\right]=\pi^{\omega}(C)
$$

Using Dynkin's lemma and standard extension techniques, this implies that

$$
\mu \text {-a.s., } \begin{cases}\pi^{\omega} \gamma_{\Lambda}(A)=\pi^{\omega}(A), & \forall \Lambda \in \mathcal{S}, \forall A \in \mathcal{F} . \\ \pi^{\omega}(B)=\mathbf{1}_{B}(\omega), & \forall B \in \mathcal{F}_{\infty} .\end{cases}
$$

In particular, one gets (2.50) and (2.51) and Lemma 2.49 is proved.

Step 2: Concentration on the extremal measures

Consider now, for $\mu \in \mathcal{G}(\gamma), \pi^{\cdot}$ as a random measure on the probability space $(\Omega, \mathcal{F}, \mu)$, taking values in the set $\mathcal{M}_{1}^{+}(\Omega)$. By the former lemma, it $\mu$-concentrates on $\mathcal{G}(\gamma)$ and has some specific tail-measurable properties 
that are useful to relate it to extremal measures. One gets then the starting point of the decomposition by rewriting (2.47) for $\mu \in \mathcal{G}(\gamma)$ :

$$
\forall A \in \mathcal{F}, \mu(A)=\int_{\Omega} \pi^{\omega}(A) d \mu(\omega)=\mu\left[\pi^{\cdot}(A)\right] .
$$

Denote formally $\alpha_{\mu}$ the law of $\pi^{\cdot}$ as a random variable on the probability space $(\Omega, \mathcal{F}, \mu)$. Writing $M_{0}=\pi^{*}(\Omega)$, one can rewrite formally (2.53) under the form

$$
\mu(\cdot)=\int_{\Omega} \pi \cdot d \mu=\int_{M_{0}} \nu \mu[\pi \cdot \in d \nu]=\int_{M_{0}} \nu \alpha_{\mu}[d \nu] .
$$

Before focusing more properly on a rigorous definition of the weights $\alpha_{\mu}$ that leads to the correct decomposition, we first establish an extra important consequence of the previous lemma: The limiting procedure used to define $\pi^{\prime}$ on the space $\Omega_{\mu}$ of full $\mu$-measure allows interesting probabilistic properties of the measure $\pi^{\omega}$ for such a typical $\omega$ : The measure $\pi^{\omega}$ is an extreme element of $\mathcal{G}(\gamma)$ and the above integral reduces to the set $M_{0}=\operatorname{ex} \mathcal{G}(\gamma)$.

\section{Lemma 2.55.}

$$
\left\{\pi^{\cdot} \in \operatorname{ex} \mathcal{G}(\gamma)\right\} \in \mathcal{F}_{\infty} \text { and }, \forall \mu \in \mathcal{G}(\gamma), \mu\left[\pi^{\cdot} \in \operatorname{ex} \mathcal{G}(\gamma)\right]=1
$$

Proof: Firstly, recall that the consistency has a consequence on the expected value of $\pi^{\cdot}(A)$ as a random variable (with values in $[0,1]$ ) on $(\Omega, \mathcal{F}, \mu)$, as seen in $(2.53): \forall A \in \mathcal{F}$,

$$
\begin{aligned}
\mu\left[\pi^{\cdot}(A)\right] & =\int_{\Omega} \pi^{\omega}(A) d \mu(\omega) \\
& =\int_{\Omega} \mu\left[A \mid \mathcal{F}_{\infty}\right](\omega) d \mu(\omega)=\mu\left[\mu\left[A \mid \mathcal{F}_{\infty}\right]\right]=\mu(A)
\end{aligned}
$$

so the expected value of $\pi^{\cdot}(A)$ is $\mu(A)$, while its variance under $\mu \in \mathcal{G}(\gamma)$ is:

$$
\begin{aligned}
\mathbb{E}_{\mu}\left[\left(\pi^{\cdot}(A)-\mu(A)\right)^{2}\right] & =\mu\left[\left(\pi^{\cdot}(A)\right)^{2}-2 \mu(A) \pi^{\cdot}(A)+(\mu(A))^{2}\right](2.56) \\
& =\mu\left[\left(\pi^{\cdot}(A)\right)^{2}\right]-2 \mu(A) \mu\left[\pi^{\cdot}(A)\right]+(\mu(A))^{2}
\end{aligned}
$$

in such a way that, when $\mu \in \mathcal{G}(\gamma)$, we can define this variance to be

$$
\sigma_{A}^{2}(\mu):=\mu\left[\left(\pi^{\cdot}(A)\right)^{2}\right]-\left[\mu\left(\pi^{\cdot}(A)\right)\right]^{2}=\mu\left[\left(\pi^{\cdot}(A)\right)^{2}\right]-\left(\mu(A)^{2}\right)
$$

or

$$
\sigma_{A}^{2}(\mu)=e_{\left(\pi^{\cdot}(A)\right)^{2}}(\mu)-\left(e_{\pi^{\cdot}(A)}(\mu)\right)^{2}
$$


that could in particular be used to get (tail) measurability. For technical reasons, we extend the definition of this map $\sigma_{A}^{2}$ on the whole space $\mathcal{M}_{1}^{+}(\Omega)$ using $^{16}$ the same expression (2.58).

By its definition via $(2.46), \pi^{\cdot}(A)$ is a version of $\mu\left[A \mid \mathcal{F}_{\infty}\right]$ for all $A \in \mathcal{F}$ and by (2.44) $\mu$ is extreme if and only if $\mu\left[A \mid \mathcal{F}_{\infty}\right]=\mu(A), \mu$-a.s., $\forall A \in \mathcal{F}$. This implies that the starting DLR measure $\mu \in \mathcal{G}(\gamma)$ will be extreme if and only if

$$
\forall A \in \mathcal{F}, \pi^{\cdot}(A)=\mu(A), \mu-\text { a.s. }
$$

in such a way that

$$
\operatorname{ex} \mathcal{G}(\gamma)=\left\{\mu \in \mathcal{G}(\gamma): \pi^{\cdot}(A)=\mu(A) \mu \text {-a.s., } \forall A \in \mathcal{F}\right\} \text {. }
$$

Using Dynkin's lemma for the $\mathcal{D}$-system $\left\{A \in \mathcal{F}: \pi^{\cdot}(A)=\mu(A) \mu\right.$-a.s. $\}$ that contains $\mathcal{C}$, one gets

$$
\left\{\pi^{\cdot} \in \operatorname{ex} \mathcal{G}(\gamma)\right\}=\left\{\pi^{\cdot} \in \mathcal{G}(\gamma)\right\} \cap \bigcap_{C \in \mathcal{C}}\left\{\pi^{\cdot}: \pi^{\cdot}(C)=\mu(C)\right\}
$$

which in particular insures the $\mathcal{F}_{\infty}$-measurability of $\left\{\pi^{\cdot} \in \operatorname{ex} \mathcal{G}(\gamma)\right\}$ from (2.50) and from this of $\pi^{\circ}(C)$ for any $C \in \mathcal{C}$. Hence, the extremal measures are $\mu \in \operatorname{ex} \mathcal{G}(\gamma)$ satisfy

$$
\forall C \in \mathcal{C}, \pi^{\cdot}(C)=\mu(C), \mu-\text { a.s. }
$$

i.e. that for all $C \in \mathcal{C}, \pi^{\cdot}(C)$ would be, as a random variable, almost surely equal to its $\mu$-expectation. As in many cases in such situations, it implies that its variance $(2.57)$ should be $\mu$-a.s. zero:

$$
\mu \in \operatorname{ex} \mathcal{G}(\gamma) \Longleftrightarrow \mu \in \mathcal{G}(\gamma) \text { and } \forall C \in \mathcal{C}, \mu\left[\sigma_{C}^{2}\left(\pi^{\cdot}(C)\right)=0\right]=1 .
$$

This proves that $\pi^{\cdot}$ is itself extreme $\mu$-almost surely, as a consequence of the tail measurability of the random variable $\pi^{*}: A \longmapsto \pi^{*}(A)$. Indeed, one then has $\mu\left[\left(\pi^{\cdot}(A)\right)^{2} \mid \mathcal{F}_{\infty}\right]=\left(\pi^{\cdot}(A)\right)^{2} \mu$-a.s., for all $A \in \mathcal{F}$, and in particular, for all $C \in \mathcal{C}$,

$$
\mu\left[\mu\left[\left(\pi^{\cdot}(C)\right)^{2} \mid \mathcal{F}_{\infty}\right](\omega)-\left(\pi^{\omega}(C)\right)^{2}\right]=0
$$

which implies

$$
\mu\left[\sigma_{\pi}^{2} \cdot\left(\pi^{\cdot}(C)\right)^{2}\right]=\mu\left[\pi^{\omega}\left[\left(\pi^{\cdot}(C)\right)^{2}\right]-\left(\pi^{\omega}(C)\right)^{2}\right]=0 .
$$

This proves $\mu\left[\pi^{\cdot} \in \operatorname{ex} \mathcal{G}(\gamma)\right]=1$ and the lemma using (2.60).

\footnotetext{
${ }^{16}$ Instead of the more usual variance (2.56). The two expressions coincide on $\mathcal{G}(\gamma)$.
} 


\section{Step 3: Extreme decomposition and its uniqueness}

To properly get the decomposition using the concentration of the asymptotic kernels on the extreme DLR measures, we use the tail-measurability of $\pi^{\cdot}$ got in the previous lemma together with the very definition of the conditional expectation in an extended version of (2.47):

$$
\forall A \in \mathcal{F}, \forall B \in \mathcal{F}_{\infty}, \int_{B} \mu\left[A \mid \mathcal{F}_{\infty}\right](\cdot) d \mu(\cdot)=\int_{B} \mathbf{1}_{A}(\cdot) d \mu(\cdot) .
$$

By Lemma 2.55, $B=\left\{\pi^{\cdot} \in \operatorname{ex} \mathcal{G}(\gamma)\right\} \in \mathcal{F}_{\infty}$ so in particular, one has for all $A \in \mathcal{F}$,

$$
\begin{aligned}
\int_{\{\pi \cdot \in \operatorname{ex} \mathcal{G}(\gamma)\}} \mu\left[A \mid \mathcal{F}_{\infty}\right](\cdot) d \mu(\cdot) & =\int_{\{\pi \cdot \in \operatorname{ex} \mathcal{G}(\gamma)\}} \mathbf{1}_{A}(\cdot) d \mu(\cdot) \\
& =\mu\left(A \cap\left\{\pi^{\cdot} \in \operatorname{ex} \mathcal{G}(\gamma)\right\}\right)
\end{aligned}
$$

and the latter is exactly $\mu(A)$ by concentration of $\pi^{\cdot}$ on $\operatorname{ex} \mathcal{G}(\gamma)$ for $\mu \in$ $\mathcal{G}(\gamma)$. Thus one can rewrite $(2.53)$ as

$$
\begin{aligned}
\mu(\cdot)=\mu\left(\cdot \cap\left\{\pi^{\cdot} \in \operatorname{ex} \mathcal{G}(\gamma)\right\}\right) & =\int_{\left\{\pi^{\cdot} \in \operatorname{ex} \mathcal{G}(\gamma)\right\}} \mu\left[\cdot \mid \mathcal{F}_{\infty}\right](\omega) d \mu(\omega) \\
& =\int_{\left\{\pi^{\cdot} \in \operatorname{ex} \mathcal{G}(\gamma)\right\}} \pi^{\omega}(\cdot) d \mu(\omega) .
\end{aligned}
$$

Consider $\alpha_{\mu}$ as defined by (2.30) and extend it into a probability measure $\alpha_{\mu} \in \mathcal{M}_{1}^{+}(\operatorname{ex} \mathcal{G}(\gamma), e(\operatorname{ex} \mathcal{G}(\gamma))$, defined to be the law of $\pi$ as a random extremal DLR measure, i.e. by

$$
\alpha_{\mu}(M):=\mu\left(\pi^{\cdot} \in M\right), \quad \forall M \in e(\operatorname{ex} \mathcal{G}(\gamma)) .
$$

It is indeed a probability measure because for all $M \in e(\operatorname{ex} \mathcal{G}(\gamma))$ one has $\left\{\pi^{\cdot} \in M\right\} \in \mathcal{F}_{\infty} \subset \mathcal{F}$, and because $\alpha_{\mu}(\operatorname{ex} \mathcal{G}(\gamma))=1$ by step 2 . above ${ }^{17}$. Thus we identify $M_{0}$ by $\operatorname{ex} \mathcal{G}(\gamma)$ in (2.54) and rewrite (2.63) under the form

$$
\mu=\int_{\operatorname{ex\mathcal {G}(\gamma )}} \nu \alpha_{\mu}(d \nu)
$$

Uniqueness of the representation follows by the uniqueness of the extension in the core property and from the uniqueness of the representation of a probability measure via its action on measurable functions. Theorem 2.31 is thus proved.

\footnotetext{
${ }^{17}$ Rigorously speaking, one should use the expression (2.58) in terms of the evaluation maps to prove the measurability on $(\operatorname{ex} \mathcal{G}(\gamma), e(\operatorname{ex} \mathcal{G}(\gamma)))$, see e.g. [52].
} 


\subsubsection{Selections by boundary conditions}

In statistical physics, Gibbs measures are often considered by taking the infinite-volume limit of finite-volume specifications with prescribed boundary conditions. This is not rigorously true for general DLR measures, but a corollary of the simplicial decomposition indeed indicates that it is true for extremal measures ${ }^{18}$. The direct description of non-extremal ones is more peculiar, but of course can be done using this decomposition, see [52, 36] for more details. Stronger results are also true in the quasilocal context, but the latter is not necessary for what follows; it is important for us while we shall consider non-quasilocal measures within the still active Dobrushin program of restoration of Gibbsianness. Extreme points have thus the nice general extra property to get identified with some particular sequence of measures with boundary conditions: An infinite-volume extremal measure specified by $\gamma$ can be selected by a sequences of finite-volume measures with boundary conditions that are typical for it:

Theorem 2.65. [52] Let $\gamma$ be a specification s.t. there exists $\mu \in \operatorname{ex} \mathcal{G}(\gamma)$. Then, for any sequence of cubes $\left(\Lambda_{n}\right) \in \mathcal{S}$, for any $f \in \mathcal{F}$ bounded, the following convergence holds:

$$
\begin{array}{ccc}
\gamma_{\Lambda_{n}} f(\cdot) & \underset{n \rightarrow \infty}{\longrightarrow} \mu[f], \quad \mu-\text { a.s. } \\
\gamma_{\Lambda_{n}}(\cdot \mid \omega) & \underset{n \rightarrow \infty}{W} & \mu(\cdot), \text { for } \mu-\text { a.e. }(\omega) .
\end{array}
$$

In case of phase transitions, it provides a more explicit description of extremal measures:

Theorem 2.68. [52] Let $\gamma$ be a specification s.t. there exists $\mu \neq \nu \in$ $\operatorname{ex\mathcal {G}}(\gamma)$. Consider $f \in \mathcal{F}$ bounded such that $\mu[f] \neq \nu[f]$. Then the tailmeasurable sets

$$
\begin{aligned}
B_{\mu}^{f} & =\left\{\omega: \gamma_{\Lambda_{n}} f(\omega) \underset{n \rightarrow \infty}{\longrightarrow} \mu[f]\right\} \\
B_{\nu}^{f} & =\left\{\omega: \gamma_{\Lambda_{n}} f(\omega) \underset{n \rightarrow \infty}{\longrightarrow} \nu[f]\right\}
\end{aligned}
$$

are such that $\mu\left(B_{\mu}^{f}\right)=\nu\left(B_{\nu}^{f}\right)=1$ and $\nu\left(B_{\mu}^{f}\right)=\mu\left(B_{\nu}^{f}\right)=0$.

\section{Basic example: 2d Ising model at low temperature:}

Although the simplicial representation is a very satisfactory result from a theoretical point of view, it is far from being an easy task to characterize and describe the extreme DLR measures for a given specification. We shall

\footnotetext{
${ }^{18}$ The converse statement is not true. There exists non extremal measures that are such weak limits, see e.g. the 3 -states Potts models with well-chosen external field.
} 
mention a few known examples later on in this course, but we also stress here that the description is still mostly incomplete from the mathematical point of view. The most complete results concern the standard Ising model on $\mathbb{Z}^{2}$. For this model, we have seen in Theorem 2.27 that the set of DLR measures is the convex set $\left[\mu_{\beta}^{-}, \mu_{\beta}^{+}\right]$where the extremal measures are characterized by an opposite magnetization $\pm m_{\beta} \in[0,1]$, defined to be $m_{\beta}=\mathbb{E}_{\mu_{\beta}^{+}}\left[\sigma_{0}\right]=-\mathbb{E}_{\mu_{\beta}^{-}}\left[\sigma_{0}\right]$, with $m_{\beta} \neq 0$ at low enough temperature. In such a case, one can write for any $\mu \in \mathcal{G}(\gamma)$, using (2.64) and the definition (2.34) of the weights,

$$
\mu=\alpha_{\mu}\left(\left\{\mu_{\beta}^{+}\right\}\right) \cdot \mu_{\beta}^{+}+\alpha_{\mu}\left(\left\{\mu_{\beta}^{-}\right\}\right) \cdot \mu_{\beta}^{-}
$$

where the weights can be shown to satisfy

$$
\alpha_{\mu}\left(\mu_{\beta}^{ \pm}\right)=\mu\left[\left\{\omega \in \Omega: \lim _{n} \gamma_{\Lambda_{n}}(C \mid \pm)=\mu_{\beta}^{ \pm}(C) \text { for any } C \in \mathcal{C}\right\}\right]=\mu\left(B_{m_{\beta}^{ \pm}}\right)
$$

with the sets $B_{m}$ defined in $(2.2)$ for $m \in[0,1]$. For the Gibbs measure with free boundary conditions ${ }^{19}$, one e.g. recovers

$$
\mu^{f}=\frac{1}{2} \cdot \mu_{\beta}^{+}+\frac{1}{2} \cdot \mu_{\beta}^{-} .
$$

\subsubsection{Ergodic vs. extremal DLR measures}

Thus, Theorem 2.31 tells us that for a given specification, any DLR measure is uniquely determined in terms of the extremal ones, those that are trivial on the tail $\sigma$-algebra $\mathcal{F}_{\infty}$, i.e. for which global macroscopic observables do not fluctuate. This is the reason why they are related to macroscopic states of our system, as we shall discuss soon. This description is reminiscent to the standard ergodic decomposition of translation-invariant probability measures. Let us describe briefly the proof of [52] that gets this decomposition as a corollary of the previous theorem, using a particular specification related to a spatial average operator.

Hence, as usual in ergodic theory, we focus now on the set $\mathcal{M}_{1, \text { inv }}^{+}(\Omega)$ of translation-invariant probability measures and on the $\sigma$-algebra $\mathcal{F}_{\text {inv }}$ of translation-invariant events. Introducing a particular specification $\tilde{\gamma}$ defined for all $A \in \mathcal{F}, \omega \in \Omega$ and $\Lambda \in \mathcal{S}$ by

$$
\tilde{\gamma}_{\Lambda}(A \mid \omega)=\frac{1}{|\Lambda|} \sum_{i \in \Lambda} \mathbf{1}_{A}\left(\tau_{i} \omega\right)
$$

one easily gets that the $\tilde{\gamma}$-invariant sets are exactly the translation-invariant ones, i.e.

$$
\mathcal{F}_{\tilde{\gamma}}=\mathcal{F}_{\text {inv }} .
$$

${ }^{19}$ I.e. without any boundary condition, see in next section. 
By an adaptation of the proof of the extreme decomposition (2.63), in the more general framework of [31], one gets the following theorem, proved in Chapter 14 of [52]:

Theorem 2.69. [52, 36] The set $\mathcal{M}_{1, \text { inv }}^{+}(\Omega)$ is a convex subset of $\mathcal{M}_{1}^{+}(\Omega)$ such that:

1. its extreme elements are the probability measures that are trivial on the translation-invariant $\sigma$-algebra $\mathcal{F}_{\text {inv }}$, i.e. the ergodic probability measures on $(\Omega, \mathcal{F})$ :

$$
\operatorname{erg}(\Omega)=\left\{\mu \in \mathcal{M}_{1, \text { inv }}^{+}(\Omega): \mu(A)=0 \text { or } 1, \forall A \in \mathcal{F}_{\text {inv }}\right\} .
$$

Distinct ergodic measures $\mu, \nu$ are mutually singular: $\exists A \in \mathcal{F}_{\text {inv }}$, $\mu(A)=1$ and $\nu(A)=0$, and more generally, each $\mu \in \operatorname{erg}(\Omega)$ is uniquely determined within the ergodic measures by its restriction on $\mathcal{F}_{\text {inv }}$.

2. $\mathcal{M}_{1, \mathrm{inv}}^{+}(\Omega)$ is a Choquet simplex: Any $\mu \in \mathcal{M}_{1, \mathrm{inv}}^{+}(\Omega)$ can be written in a unique way as

$$
\mu=\int_{\operatorname{erg}(\Omega)} \nu \cdot \alpha_{\mu}(d \nu)
$$

with $\alpha_{\mu} \in \mathcal{M}_{1}^{+}(\operatorname{erg}(\Omega), e(\operatorname{erg}(\Omega)))$ defined for all $M \in e(\operatorname{erg} \mathcal{M})$ by

$\alpha_{\mu}(M)=\mu\left[\left\{\omega \in \Omega: \exists \nu \in M, \lim _{n} \tilde{\gamma}_{\Lambda_{n}}(C \mid \omega)=\nu(C)\right.\right.$ for any $\left.\left.C \in \mathcal{C}\right\}\right]$.

In this theorem, translation-invariance and the ergodic theorem play the roles respectively devoted to tail-triviality and the backward martingale limit theorem in Theorem 2.31. Many other similarities exist, described e.g. in Chapter 14 of [52] or in [36].

Remark 2.71 (Physical Phases). Once we agree to describe the true physical phases of the system by some random field of $\mathcal{M}_{1, \text { inv }}^{+}(\Omega)$, we have now three different mathematical manners to characterize the macroscopic "states" of the systems we want to model. Starting from a specification describing an equilibrium at finite volume ${ }^{20}$, one can consider first the set of DLR measures $\mathcal{G}(\gamma)$ as good candidates to play the same role at infinite volume, leaving moreover the door open to the modelization of phase transitions. Requiring furthermore that macroscopic, i.e. tail-measurable, observables should not fluctuate, one can then restrict the macroscopic description to the DLR measures that are trivial on the tail $\sigma$-algebra,

\footnotetext{
${ }^{20}$ It corresponds to the finite-volume Boltzmann-Gibbs weights, see next chapter.
} 
i.e. to $\operatorname{ex} \mathcal{G}(\gamma)$, and use thereafter the extreme decomposition to get a more general description that incorporates uncertainty of the experiment. For other purposes, one can also be interested in translation-invariant objects, in particular when the underlying system is translation-invariant, and chose then to focus either on the set $\mathcal{G}_{\text {inv }}(\gamma)=\mathcal{M}_{1 \text {,inv }}^{+}(\Omega) \cap \mathcal{G}(\gamma)$ and on its extreme elements ex $\mathcal{G}_{\text {inv }}(\gamma)$, or either on the translation-invariant extremal measures, i.e. the translation-invariant elements of $\operatorname{ex} \mathcal{G}(\gamma)$. These approaches are far from being equivalent: The latter form a rather small and sometimes empty set, whereas the former consists of ergodic measures, that are in particular extreme because $\mathcal{F}_{\text {inv }} \subset \mathcal{F}_{\infty}$ in the following almost sure sense: For each $\mu \in \mathcal{M}_{1}^{+}(\Omega)$ and each $A \in \mathcal{F}_{\text {inv }}$, there exists $B \in \mathcal{F}_{\infty}$ with $\mu(A \Delta B)=0$, where $\Delta$ is the standard symmetric difference of sets. These ergodic measures are thus often chosen to be the physical phases that represent macroscopically the equilibrium state of the underlying interacting particle system. If one does not focus on translation-invariance, the structure of extremal states could be very rich and far from being equivalent to ergodicity, see various examples of Ising models at higher dimensions [26], or antiferromagnetic [36], or on trees [8, 57, 62, 107].

Remark 2.72 (de Finetti's theorem and exchangeability). Let us also mention now that within a slightly different framework, a theorem similar to Theorem 2.31 is equivalent to the de Finetti's theorem for exchangeable measures ${ }^{21}$. Instead of working on a lattice, consider $S$ to be the set of non-negative integers $\mathbb{N}$, and focus on permutations and its associate $\sigma$-algebra of symmetric events $\mathcal{I}=\cap_{n \in \mathbb{N}}$ already introduced in Section 2.2. This symmetric $\sigma$-algebra plays the role devoted to $\mathcal{F}_{\infty}$ in the extreme decomposition, and defining a family of proper probability kernels $\gamma=\left(\gamma_{n}\right)_{n \in \mathbb{N}}$ from $(\Omega, \mathcal{I})$ to $(\Omega, \mathcal{F})$ by,

$$
\forall n \in \mathbb{N}, \forall A \in \mathcal{F}, \forall \omega \in \Omega, \gamma_{n}(A \mid \omega)=\frac{1}{n !} \sum_{\tau \in I_{n}} \mathbf{1}_{A}(\tau \omega)
$$

one first gets that the set $\mathcal{G}(\gamma)$ of $\gamma$-invariant probability measures is exactly the set of exchangeable measures. Using a corollary of Theorem 2.31 , its extreme points have then to be trivial on the symmetric events $\mathcal{I}$, corresponding thus to the product measures of the form $\lambda^{\otimes \mathbb{N}}$ with $\lambda \in \mathcal{M}_{1}^{+}(E, \mathcal{E})$. Proceeding in a similar way as in the extreme decomposition of Theorem 2.31, one gets that all permutation-invariant (or exchangeable) measures are uniquely determined as convex combinations of product measures: This is exactly de Finetti's theorem [25]. A more refined analysis of this analogy and of the $\sigma$-algebras $\mathcal{I}, \mathcal{F}_{\text {inv }}$ and $\mathcal{F}_{\infty}$ also leads to related $0-1$ laws, see $[3,4,31,52]$.

\footnotetext{
${ }^{21}$ See e.g. [25]. It can also be used to complete the description of mean-field models, see e.g. the discussion in [83].
} 


\section{Chapter 3}

\section{Quasilocal and Gibbs measures}

We recall that in general in this volume, we restrict our studies to the case of finite single-spin state-space and we moreover emphasize that, in this chapter in particular, some of the results or equivalences are not true if this hypothesis is not fulfilled, see e.g. Proposition 3.5.

\subsection{Quasilocality for measures and specifica- tions}

\subsubsection{Essential continuity of conditional probabilities}

The link between continuity and quasilocality described through the product topology enables to generalize the Markov property to probability measures whose conditional expectations of local functions depend only weakly of spins arbitrarily far away from their support. This leads to the concept of quasilocal measures, which, in addition to provide a good framework to get the existence of specified measures, is also closely related to the notion of Gibbs measures. In our settings, it corresponds to the concept of Feller kernels in the standard theory of stochastic processes.

Definition 3.1. A specification $\gamma$ is said to be quasilocal when

$$
\forall \Lambda \in \mathcal{S}, \quad f \in \mathcal{F}_{\text {loc }} \Longrightarrow \gamma_{\Lambda} f \in \mathcal{F}_{\text {qloc }} .
$$

A measure is said to be quasilocal if there exists a quasilocal specification $\gamma$ such that $\mu \in \mathcal{G}(\gamma)$. 
Recall that for any $\Lambda \in \mathcal{S}, \gamma_{\Lambda} f$ is defined by:

$$
\forall \omega \in \Omega, \gamma_{\Lambda} f(\omega)=\int_{\Omega} f(\sigma) \gamma_{\Lambda}(d \sigma \mid \omega) .
$$

Thus, $\gamma_{\Lambda}$ being properly speaking a kernel from $\left(\Omega_{\Lambda^{c}}, \mathcal{F}_{\Lambda^{c}}\right)$ to $\left(\Omega_{\Lambda}, \mathcal{F}_{\Lambda}\right)$, the function $\gamma_{\Lambda} f$ is $\mathcal{F}_{\Lambda^{c}-\text { measurable and continuity is to be understood }}$ here as continuity w.r.t. the boundary condition $\omega$ (depending only on $\left.\omega_{\Lambda^{c}}\right)$ : If $\gamma$ is a quasilocal, then for any $f \in \mathcal{F}_{\text {loc }}$, for any $\Lambda \in \mathcal{S}$,

$$
\lim _{\Lambda^{\prime} \uparrow \mathcal{S}} \sup _{\sigma_{\Lambda^{\prime c}}=\omega_{\Lambda^{\prime c}}}\left|\gamma_{\Lambda} f(\omega)-\gamma_{\Lambda} f(\sigma)\right|=0 .
$$

An important consequence is that the conditional probabilities of local or quasilocal functions w.r.t. a quasilocal measure are essentially continuous in the sense that for $\mu \in \mathcal{G}(\gamma)$ with $\gamma$ quasilocal, for all $f \in \mathcal{F}_{\text {qloc }}$, for all $\Lambda \in \mathcal{S}$ and $\omega \in \Omega$, there always exists a version of the conditional probability $\mu\left[f \mid \mathcal{F}_{\Lambda^{c}}\right](\cdot)$ that is continuous at $\omega$. In particular, for a given quasilocal measure $\mu$, it is not possible to change a version of conditional probabilities to make it discontinuous: Take e.g. $f(\sigma)=\sigma_{0}$, one should have for all $\Lambda \in \mathcal{S}$ and for all $\omega \in \Omega$,

$$
\lim _{\Lambda^{\prime} \uparrow \mathcal{S}} \sup _{\omega^{1}, \omega^{2} \in \Omega}\left|\mu\left[\sigma_{0} \mid \omega_{\Lambda^{\prime} \backslash \Lambda} \omega_{\Lambda^{\prime c}}^{1}\right]-\mu\left[\sigma_{0} \mid \omega_{\Lambda^{\prime} \backslash \Lambda} \omega_{\Lambda^{\prime c}}^{2}\right]\right|=0
$$

because the former conditionings are in open neighborhoods of $\omega$, and open neighborhoods are automatically of positive $\mu$-measures here [43]. This also express an almost sure asymptotic weak dependence in the conditioning that can be seen as an asymptotic extension of Markov properties, as suggested by the denomination almost Markovian chosen by Sullivan [117].

The failure of this essential continuity (3.2) will be very important in the last chapter when dealing with transformations of Gibbs or quasilocal measures and to detect non-quasilocality via essential discontinuity, characterized in the following proposition, although its formulation is a bit stronger than the usual general meaning (see [43]).

Proposition 3.3 (Essential discontinuity). A conditional probability of a measure $\mu \in \mathcal{M}_{1}^{+}(\Omega, \mathcal{F})$ is essentially discontinuous at $\omega$ if there exist $\Lambda \in \mathcal{S}, f \in \mathcal{F}_{\text {loc }}, \delta>0$ and $\mathcal{N}_{\Lambda}^{1}(\omega), \mathcal{N}_{\Lambda}^{2}(\omega)$ neighborhoods of $\omega$ such that

$$
\forall \omega^{1} \in \mathcal{N}_{\Lambda}^{1}(\omega), \omega^{2} \in \mathcal{N}_{\Lambda}^{2}(\omega),\left|\mu\left[f \mid \mathcal{F}_{\Lambda^{c}}\right]\left(\omega^{1}\right)-\mu\left[f \mid \mathcal{F}_{\Lambda^{c}}\right]\left(\omega^{2}\right)\right|>\delta
$$

or equivalently

$$
\lim _{\Lambda^{\prime} \uparrow \infty} \sup _{\omega^{1}, \omega^{2} \in \Omega}\left|\mu\left[f \mid \mathcal{F}_{\Lambda^{c}}\right]\left(\omega_{\Lambda^{\prime}} \omega_{\Lambda^{\prime c}}^{1}\right)-\mu\left[f \mid \mathcal{F}_{\Lambda^{c}}\right]\left(\omega_{\Lambda^{\prime}} \omega_{\Lambda^{\prime c}}^{2}\right)\right|>\delta .
$$




\subsubsection{Existence results in the quasilocal framework}

In our finite single-spin state-space settings, where compactness holds, quasilocality ensures thus the existence of a measure in $\mathcal{G}(\gamma)$. The following proposition additionally indicates how one can naturally construct such objects as the limit of large but finite systems with some specified boundary conditions, that have then to be typical for the measure constructed. The set of DLR measures is then a closed convex subset, and this explains the usual introduction of Gibbs measures as weak limits of finite-volume probability measures with boundary conditions. For any specification $\gamma$ and sequence $\left(\nu_{n}\right)_{n \in \mathbb{N}} \in \mathcal{M}_{1}^{+}(\Omega)$, we recall that $\nu_{n} \gamma_{n}$ denotes the probability measure acting on a bounded function $f \in \mathcal{F}$ via:

$$
\nu_{n} \gamma_{\Lambda_{n}}[f]=\int_{\Omega} \gamma_{\Lambda_{n}} f(\omega) \nu_{n}(d \omega), \forall n \in \mathbb{N} .
$$

Proposition 3.5. [52] Let $\gamma=\left(\gamma_{\Lambda}\right)_{\Lambda \in \mathcal{S}}$ be a quasilocal specification on it. Then, for any sequences of cubes $\left(\Lambda_{n}\right)_{n \in \mathbb{N}}$ and any arbitrary sequence $\left(\nu_{n}\right)_{n \in \mathbb{N}}$ on $\mathcal{M}_{1}^{+}(\Omega)$, the weak limit

$$
\mu:=\lim _{n \rightarrow \infty} \nu_{n} \gamma_{n}
$$

exists in $\mathcal{M}_{1}^{+}(\Omega)$ and $\mu \in \mathcal{G}(\gamma)$. In particular, $\mathcal{G}(\gamma)$ is a non empty convex subset of $\mathcal{M}_{1}^{+}(\Omega)$.

We introduce now the main example of quasilocal measures, that are nothing but (infinite-volume) Gibbs measures, and explain why a converse statement telling that most quasilocal measures are Gibbs is also true.

\subsection{Infinite-volume Gibbs measures}

\subsubsection{Equilibrium states at finite volume}

We recall here briefly some elementary physical concepts that led Boltzmann and Gibbs to settle down their prescription for equilibrium states. This is very simplified, and probably too simple from a physical point of view (the notion of entropy being for example far from being so simple, see e.g. $[88,80,54])$ but we state it in order to formally justify the notion of equilibrium states developed in Chapter 4 within the so-called variational principle. Hence, our aim is to provide a probabilistic translation of the second law of thermodynamics that claims:

Equilibrium at a fixed value of energy maximizes entropy

or, in an equivalent statement, Equilibrium minimizes free energy. 
For a modelization at finite volume $\Lambda$, the microscopic states are the collections $\sigma_{\Lambda} \in \Omega_{\Lambda}$ of random variables $\left(\sigma_{i}\right)_{i \in \Lambda}$ and the macroscopic states are their possible distributions $\mu_{\Lambda} \in \mathcal{M}_{1}^{+}\left(\Omega_{\Lambda}, \mathcal{F}_{\Lambda}\right)$. The energy of a configuration is represented by an Hamiltonian at finite volume $H_{\Lambda}\left(\sigma_{\Lambda}\right)$ and thus the energy of a macroscopic "state" $\mu_{\Lambda}$ is represented by the average of the Hamiltonian, i.e.

$$
\mathbb{E}_{\mu}\left[H_{\Lambda}\right]:=\sum_{\sigma_{\Lambda} \in \Omega_{\Lambda}} H_{\Lambda}\left(\sigma_{\Lambda}\right) \mu_{\Lambda}\left(d \sigma_{\Lambda}\right) .
$$

In one of its original interpretations, the entropy of a system is supposed to evaluate its degree of disorder. Translated into a probabilistic framework and quoting Khinchin in [69], "it seems highly desirable to introduce a quantity which in a reasonable way measures the amount of uncertainty associated with a given probability measure, that would be minimal for complete certainty, positive in other cases, maximal for the one with equally likely outcomes (uniform distribution), and that would have some nice monotone properties when the knowledge of the system increases". Following these ideas, one could show that such a function of a measure should involve the function $f(x)=x \ln (x)$ and the standard definition of the entropy of a (finite-volume) probability measure $\mu_{\Lambda}$ is indeed given by:

$$
\mathcal{H}_{\Lambda}(\mu)=-\sum_{\sigma_{\Lambda} \in \Omega_{\Lambda}} \mu\left(\sigma_{\Lambda}\right) \ln \mu_{\Lambda}\left(\sigma_{\Lambda}\right)
$$

In classical thermodynamics, the free energy " $F$ " of a system is usually defined through the second law in the form " $F=U-T S$ " where $U$ is the (internal) energy, $T=\frac{1}{\beta}$ the temperature and $S$ the entropy. To pursue the analogy, let us define the free energy at inverse temperature $\beta>0$ of a (finite-volume) probability measure $\mu_{\Lambda}$ to be

$$
F_{\Lambda}^{\beta}(\mu)=\mathbb{E}_{\mu}\left[H_{\Lambda}\right]-\frac{1}{\beta} \mathcal{H}_{\Lambda}(\mu) .
$$

There exist two simple ways to see which probability measures could reasonably be considered as equilibrium states, following the two different statements of the second law of thermodynamics. In the first formulation in terms of maximization of entropy, it is an elementary exercise using Lagrange's multipliers [66] to show that a probability measure $\mu_{\Lambda}$ having the given energy (3.6) and maximizing its entropy (3.7) should give weights of the form given by the famous Boltzmann-Gibbs weights $[10,51]$ to each configuration:

$$
\nu_{\Lambda}^{\beta}\left[\sigma_{\Lambda}\right]=\frac{1}{Z_{\Lambda}^{\beta}} \cdot e^{-\beta H_{\Lambda}\left(\sigma_{\Lambda}\right)}
$$

where the normalization is the partition function $Z_{\Lambda}^{\beta}=\sum_{\sigma_{\Lambda} \in \Omega_{\Lambda}} e^{-\beta H_{\Lambda}\left(\sigma_{\Lambda}\right)}$, which will be related soon to our free energy. 
To establish (3.9) and illustrate the second law of thermodynamics in its second formulation, we introduce another important concept, the relative entropy of two probability measures. For simplicity, we consider the case of probability measures $\mu_{\Lambda}$ and $\nu_{\Lambda}$ on the finite-volume configuration space $\left(\Omega_{\Lambda}, \mathcal{F}_{\Lambda}\right)$, assumed to be non-null in the sense that any configuration has a positive probability; the relative entropy of $\mu_{\Lambda}$ with respect to $\nu_{\Lambda}$ is then defined to be

$$
\mathcal{H}_{\Lambda}(\mu \mid \nu)=\sum_{\sigma_{\Lambda} \in \Omega_{\Lambda}} \mu_{\Lambda}\left(\sigma_{\Lambda}\right) \cdot \ln \frac{\mu_{\Lambda}\left(\sigma_{\Lambda}\right)}{\nu_{\Lambda}\left(\sigma_{\Lambda}\right)} .
$$

This function has among others the nice property to be non-negative for any probability measures on $\Omega_{\Lambda}$ and to be zero if and only the two measures coincide:

$$
\begin{aligned}
& \mathcal{H}_{\Lambda}(\mu \mid \nu) \geq 0 . \\
& \mathcal{H}_{\Lambda}(\mu \mid \nu)=0 \text { iff } \mu_{\Lambda}=\nu_{\Lambda} .
\end{aligned}
$$

Observing that $\mathcal{H}_{\Lambda}\left(\mu \mid \nu^{\beta}\right)=F_{\Lambda}^{\beta}(\mu)+\frac{1}{\beta} \ln Z_{\Lambda}^{\beta}$, one concludes that free energy is indeed minimal when $\mu_{\Lambda}=\nu_{\Lambda}^{\beta}$ is given by the Boltzmann-Gibbs weights (3.9). This minimal value of the free energy is then

$$
F_{\Lambda}^{\beta}\left(\nu^{\beta}\right)=-\frac{1}{\beta} \ln Z_{\Lambda}^{\beta}
$$

recovering thus the other form of the second law of thermodynamics. This justifies the following introduction of Gibbs specifications.

\subsubsection{Gibbs specifications and infinite-volume Gibbs measures}

Definition 3.10 (Potential). A potential is a family $\Phi=\left(\Phi_{A}\right)_{A \in \mathcal{S}}$ of functions

$$
\Phi_{A}: \Omega \longrightarrow \mathbb{R}
$$

indexed by the set $\mathcal{S}$ of finite subsets of $S$, such that $\forall A \in \mathcal{S}, \Phi_{A}$ is $\mathcal{F}_{A^{-}}$ measurable.

Our infinite-volume formalism incorporates the finite-volume one by considering free (or empty) boundary conditions, to extend Hamiltonians from $\Omega_{\Lambda}$ to $\Omega$ in a well defined way by considering finite sums in the following ${ }^{1}$

\footnotetext{
${ }^{1}$ This is true only when $\Phi_{A}$ is bounded for all $A \in \mathcal{S}$. In a more general framework involving "hard-core exclusion", $\Phi$ is allowed to be $\infty$ and the formalism has been adapted, see e.g. [29, 86].
} 
Definition 3.11 (Hamiltonian with free boundary condition). Consider a potential $\Phi$. For all $\Lambda \in \mathcal{S}$, the Hamiltonian at finite volume $\Lambda$ with free boundary condition associated with $\Phi$ is the well defined and $\mathcal{F}_{\Lambda}$-measurable map

$$
\begin{aligned}
\mathbf{H}_{\Lambda}^{\Phi, f}: \quad \Omega & \longrightarrow \mathbb{R} \\
& \omega \longmapsto \mathbf{H}_{\Lambda}^{\Phi, f}(\omega):=\sum_{A \in \mathcal{S}, A \subset \Lambda} \Phi_{A}(\omega) .
\end{aligned}
$$

Nevertheless, the sums involved in the Hamiltonians are not finite in general and one should focus first on convergence properties of potentials before introducing infinite-volume Hamiltonians with prescribed boundary conditions. In the following definition, convergence of series will be considered in the sense of the convergence along nets already defined: A series $\sum_{\Lambda \in \mathcal{S}} F_{\Lambda}$ converges iff the net $\left(\sum_{\Lambda \in \Delta} F_{\Lambda}\right)_{\Delta \in \mathcal{S}}$ converges to a finite limit as $\Delta \uparrow S$ in the sense of Definition 2.6. We shall illustrate this convergence in some examples soon.

Definition 3.12 (Convergence of potentials). A potential $\Phi$ is said to be

1. Nearest-neighbor iff for all $\omega \in \Omega, \Phi_{A}(\omega)=0$ unless $A=\{i\}$ or $A$ is a pair $\langle i j\rangle$ of nearest neighbors.

2. Finite-range iff there exists a range $R \in \mathbb{N}^{*}$ such that, for all $\omega$, $\Phi_{A}(\omega)=0$ for all $A$ such that $|A|>R$, where $|A|=\sup _{i, j \in A} d(i, j)$ is the diameter of $A$.

3. (Point-wise) convergent at $\omega \in \Omega$ if, for all $\Lambda \in \mathcal{S}$, the Hamiltonian

$$
\mathbf{H}_{\Lambda}^{\Phi}(\omega):=\sum_{A \in \mathcal{S}, A \cap \Lambda \neq \emptyset} \Phi_{A}(\omega)
$$

exists, convergent when the convergence holds for all $\omega \in \Omega$ and almost-surely convergent when there exists $\mu \in \mathcal{M}_{1}^{+}(\Omega)$ such that $\Phi$ is convergent at $\mu$-a.e. $\omega \in \Omega$.

4. Uniformly convergent when the series defining (3.13) are uniformly convergent in $\omega \in \Omega$, or equivalently when

$$
\lim _{\Delta \uparrow \mathcal{S}} \sup _{\omega \in \Omega}\left|\sum_{A \in \mathcal{S}, A \cap \Lambda \neq \emptyset, A \cap \Delta^{c} \neq \emptyset} \Phi_{A}(\omega)\right|=0 .
$$

5. Uniformly absolutely convergent (UAC) when

$$
\forall i \in S, \sum_{A \in \mathcal{S}, A \ni i} \sup _{\omega \in \Omega}\left|\Phi_{A}(\omega)\right|<+\infty .
$$


Nearest-neighbor and finite-range potentials are UAC and obviously

Lemma 3.16. $\Phi U A C \Longrightarrow \Phi$ uniformly convergent $\Longrightarrow \Phi$ convergent.

A potential that is UAC satisfies also, for any $\Lambda \in \mathcal{S}$,

$$
\sum_{A \in \mathcal{S}, A \cap \Lambda \neq \emptyset} \sup _{\omega \in \Omega}\left|\Phi_{A}(\omega)\right|<+\infty
$$

which in particular implies uniform convergence (it corresponds to normal convergence of series).

Remark 3.17. If we do not make precise the way these infinite sums are done, the sum $\mathbf{H}_{\Lambda}^{\Phi}$ in (3.13) could be ill-defined. Consider the pair (but not n.n.) potential $\Phi$ defined for all $\omega \in\{-1,+1\}^{\mathbb{Z}}$ by $\Phi_{A}(\omega)=\frac{1}{|i-j|} \omega_{i} \omega_{j}$ if $A=\{i, j\}$ and $\Phi_{A}=0$ when $A$ is not a pair ${ }^{2}$. Let $\Lambda \in \mathcal{S}, \omega \in \Omega$, write $S=B^{+} \cup B^{-}$, with $B^{ \pm}=B^{ \pm}(\omega)=\left\{i \in \mathbb{Z}, \omega_{i}= \pm 1\right\}$ to get for $A \in \mathcal{S}$

$$
\begin{array}{r}
\sum_{A \cap \Lambda \neq \emptyset} \Phi_{A}(\omega)=\sum_{A \cap \Lambda \neq \emptyset, A \subset B^{+}(\omega)} \Phi_{A}(\omega)+\sum_{A \cap \Lambda \neq \emptyset, A \subset B^{-}(\omega)} \Phi_{A}(\omega) \\
+\sum_{A \cap \Lambda \neq \emptyset, A \cap B^{+}(\omega) \neq \emptyset, A \cap B^{-}(\omega) \neq \emptyset} \Phi_{A}(\omega) .
\end{array}
$$

But

$$
\sum_{A \cap \Lambda \neq \emptyset, A \subset B^{+}(\omega)} \Phi_{A}(\omega)=\sum_{A \cap \Lambda \neq \emptyset, A \subset B^{-}(\omega)} \Phi_{A}(\omega)=\sum_{i \in \Lambda, j \in \mathbb{Z}} \frac{1}{|i-j|}
$$

are non-convergent series $^{3}$ whereas the series

$$
\sum_{A \cap \Lambda \neq \emptyset A \cap B^{+}(\omega) \neq \emptyset, A \cap B^{-}(\omega) \neq \emptyset} \Phi_{A}(\omega)
$$

can be convergent for some $\omega$ 's. Thus, the series could be non-convergent whereas $\mathbf{H}_{\Lambda}^{\Phi}$ is well-defined if we use nets as above.

\section{Examples of potentials}

1. Ising potentials: The single-spin state-space is $E=\{-1,+1\}$ with a priori measure $\rho_{0}=\frac{1}{2} \delta_{-1}+\frac{1}{2} \delta_{+1}$. The (n.n.) Ising potential is $\Phi=\left(\Phi_{A}\right)_{A \in \mathcal{S}}$ defined by

$$
\Phi_{A}(\omega)= \begin{cases}-J(i, j) \cdot \omega_{i} \cdot \omega_{j} & \text { if } A=\{i, j\} \\ -h(i) \cdot \omega_{i} & \text { if } A=\{i\} \\ 0 & \text { otherwise }\end{cases}
$$

\footnotetext{
${ }^{2}$ This potential corresponds to the so-called Coulomb interactions, see e.g. [113].

${ }^{3}$ Although the general term depends on $\omega$, the l.h.s is independent of it, because of the particular way the sum has been split.
} 
where $J: S \times S \longrightarrow \mathbb{R}$ is called the coupling function and $h: S \longrightarrow \mathbb{R}$ the external magnetic field. In the standard Ising model, $J(i, j)=0$ unless $i, j$ are n.n. and when both $J$ and $h$ are constant, we call it homogeneous Ising model, inhomogeneous otherwise. It is said to be ferromagnetic when $J \geq 0$ and anti-ferromagnetic when $J<0$. Less standard non-n.n. Ising models are also sometimes considered. One studies e.g. long-range Ising models where $J(i, j)=\frac{1}{|i-j|^{r}}$ for any pair $\{i, j\}$, well defined for $r>d$ when $S=\mathbb{Z}^{d}$, which exhibits a phase transition in one dimension for $r \in] 1,2$ ] (see in next sections for a few results), or so-called Kac-Ising potentials which have a long but finite range. Their origin comes from a description of the van der Waals theory of liquid-vapor transitions initiated by [67] and we follow here the terminology of [22]. The starting point is a smooth non-negative function supported by the unit ball $J(\cdot)$ and normalized as a probability kernel (i.e. $\|J\|_{1}=1$ ). The Kac interaction allows ranges from 1 to $+\infty$ via a parameter $\gamma>0$ and coupling constants

$$
J_{\gamma}(i, j)=\gamma^{d} \cdot J(\gamma|i-j|), \quad \forall i, j \in S
$$

In the original van der Waals theory, one is particularly interested in small $\gamma$ for which the model presents a long but finite range interaction (of order $\gamma^{-1}$ ), small coupling constants (of order $\gamma^{d}$ ) and a total strength at each site of constant order 1 , and in performing thereafter the limit $\gamma$ goes to 0 to approach mean-field models. It corresponds to the n.n. Ising model when $\gamma=1$, and thus Kac models allow an interplay between n.n. and mean-field models.

\section{A (uniformly) convergent potential that is not UAC:}

This example is due to Sullivan [117]. Consider $\Omega=\{-1,+1\}^{\mathbb{Z}}$ and define a potential $\Phi$ that is non-null only for the finite sets of adjacent sequences in $\mathbb{Z}$ on which the spins are all +1 . More precisely, it is defined for all $A \in \mathcal{S}$ and for all $\omega \in \Omega$ by $\Phi_{A}(\omega)=$ $\frac{(-1)^{n}}{n^{2}}$ iff $\omega_{i}=+1, \quad \forall i \in A=\{k, \cdots, k+n-1\}, k \in \mathbb{Z}, n \in$ $\mathbb{N}^{*}$, and by $\Phi_{A}=0$ otherwise. To prove that $\Phi$ is a convergent potential, we prove that the series $\mathbf{H}_{\Lambda}^{\Phi}(\omega)=\sum_{A \cap \Lambda \neq \emptyset, A \in \mathcal{S}} \Phi_{A}(\omega)$ are convergent for $\Lambda=\{0\}$, the extension to all finite subsets $\Lambda$ being then straightforward. This amounts to prove that, for all $\omega \in \Omega$, the sequence of general term $U_{n}(\omega)=\sum_{A \ni 0, A \cap \Lambda_{n}^{c} \neq \emptyset} \Phi_{A}(\omega)$ converges to zero when $\mathrm{n}$ goes to infinity. Here it becomes

$$
\sum_{A \ni 0, A \cap \Lambda_{n}^{c} \neq \emptyset} \Phi_{A}(\omega)=\sum_{k>n} \sum_{A \ni 0,|A|=k} \frac{(-1)^{k}}{k^{2}} \cdot \prod_{i \in A} \mathbf{1}_{\left\{\omega_{i}=+1\right\}}(\omega) .
$$


and one has for $n$ large enough, uniformly in $\omega$,

$0 \leq\left|U_{n}(\omega)\right|=\left|\sum_{A \ni 0, A \cap \Delta_{n}^{c} \neq \emptyset} \Phi_{A}(\omega)\right| \leq\left|U_{n}(+)\right|=\left|\sum_{k>n}(k+1) \frac{(-1)^{k}}{k^{2}}\right|$

where the term on the right is the tail of a convergent alternating series, which is convergent. This potential is thus uniformly convergent, but it is not uniformly absolutely convergent (UAC):

$$
\sum_{A \ni 0, A \subset \Lambda_{n}} \sup _{\omega \in \Omega}\left|\Phi_{A}(\omega)\right|=\sum_{n=0}^{2 k}(k+1)\left|\frac{(-1)^{k}}{k^{2}}\right|=\sum_{k=0}^{2 n} \frac{k+1}{k^{2}}
$$

and the latter is a non-convergent series.

3. Almost surely convergent potential: Potentials associated to renormalized measures when defined as weakly Gibbsian measures, see Chapter 5 or [97].

4. (Relatively) uniformly convergent potential: Sullivan [117] has introduced this notion of convergence slightly weaker than uniform convergence, which can be associated to any quasilocal specification and which is translation-invariant when the specification is. See in next section and Remark 3.58.

5. UAC: We describe in next section how to define a UAC potential from a quasilocal specification, following a general construction of Kozlov [72].

Before introducing Gibbs measures properly speaking, we give a general definition of particular potentials that will be used to build a convergent potential associated to a quasilocal specification in the forthcoming Theorem 3.39, and later on in Chapter 5 to establish thermodynamic properties in the generalized Gibbsian framework.

Definition 3.18 (Vacuum potential). Let $\Phi$ be a potential and denote by $+=\left(+_{i}\right)_{i \in S}$ a particular ${ }^{4}$ configuration of $\Omega$. We say that $\Phi$ is a vacuum potential with vacuum state $+\in \Omega$ iff $\Phi_{A}(\omega)=0$ whenever $\omega_{i}=$ $+_{i}$ for some $i \in A \in \mathcal{S}$.

For such a potential, also called lattice gas potential, the Hamiltonian with free boundary conditions can be seen as an Hamiltonian with the vacuum state as boundary condition, when Hamiltonians with boundary condition are defined by the following

\footnotetext{
${ }^{4}$ This could be any configuration, we denote it by "+" only by analogy with the Ising model. In particular, it is not necessarily translation-invariant.
} 
Definition 3.19 (Hamiltonian at volume $\Lambda$ with boundary condition $\omega)$. When $\Phi$ is a convergent potential, the Hamiltonian at volume $\Lambda \in \mathcal{S}$ with boundary condition $\omega \in \Omega$ is defined for all $\sigma \in \Omega$ by

$$
\mathbf{H}_{\Lambda}^{\Phi, \omega}(\sigma)=\mathbf{H}_{\Lambda}^{\Phi}(\sigma \mid \omega):=\mathbf{H}_{\Lambda}^{\Phi}\left(\sigma_{\Lambda} \omega_{\Lambda^{c}}\right)=\sum_{A \in \mathcal{S}, A \cap \Lambda \neq \emptyset} \Phi_{A}\left(\sigma_{\Lambda} \omega_{\Lambda^{c}}\right)
$$

A convergent potential is regular enough to define this Hamiltonian with boundary conditions, but it will not be enough to define Gibbs measures with the right expected properties, for which UAC is usually required. At finite volume $\Lambda$, the Hamiltonian with free boundary conditions of a configuration $\sigma$ is seen as the energy of the system contained in $\Lambda$ when it is in the configuration $\sigma$, and a UAC convergence means that a change of a configuration in a finite part of the infinite system produces always a finite change of the total energy. Requiring for a potential to be UAC will be enough to define a Gibbsian specification associated with this potential, and then to provide a "reasonable" modelling of the physical properties of the system ${ }^{5}$. This requirement actually seems to be too strong, and this possibly too strong requirement causes troubles in the analysis of some renormalization group transformations, leading to generalized Gibbs measures described in Chapter 5.

We are now ready to introduce Gibbs specifications and Gibbs measures, defined from a UAC potential. First we introduce the following normalization, central in statistical physics and related to the free energy of the system as we shall see in Chapter 4.

Definition 3.21 (Partition function). Let $\Phi$ be a convergent potential, $\omega \in \Omega, \beta>0$ and $\Lambda \in \mathcal{S}$. We call partition function at temperature $\beta^{-1}$, at volume $\Lambda$, with potential $\Phi$ and boundary condition $\omega$, the $\mathcal{F}_{\Lambda^{c}-\text { measurable }}$ function

$$
\mathbf{Z}_{\Lambda}^{\beta \Phi}(\omega)=\int_{\Omega} e^{-\beta \mathbf{H}_{\Lambda}^{\Phi}(\sigma)} \kappa_{\Lambda}(d \sigma)=\int_{\Omega_{\Lambda}} e^{-\beta \mathbf{H}_{\Lambda}^{\Phi}(\sigma \mid \omega)} \rho_{\Lambda}\left(d \sigma_{\Lambda}\right)
$$

where $\kappa_{\Lambda}=\rho_{\Lambda} \otimes \delta_{\omega_{\Lambda^{c}}}^{\otimes \Lambda^{c}} \in \mathcal{M}_{1}^{+}(\Omega)$, and $\delta_{x}$ is the Dirac measure on $x \in E^{\Lambda}$.

When free boundary conditions are considered, the partition function is denoted $Z_{\Lambda}^{\beta \Phi, \mathrm{f}}$.

Definition 3.22 (Gibbs distribution at finite volume $\Lambda$ ). Let $\Phi$ be a $U A C$ potential. For $\Lambda \in \mathcal{S}$, we call Gibbs distribution at finite volume $\Lambda$, with potential $\Phi$, at temperature $\beta^{-1}$ and with boundary condition $\omega \in \Omega$, the probability measure $\gamma_{\Lambda}^{\beta \Phi}(\cdot \mid \omega)$ on $(\Omega, \mathcal{F})$ defined by:

$$
\forall A \in \mathcal{F}, \gamma_{\Lambda}^{\beta \Phi}(A \mid \omega)=\frac{1}{\mathbf{Z}_{\Lambda}^{\beta \Phi}(\omega)} \int_{\Omega_{\Lambda}} \mathbf{1}_{A}\left(\sigma_{\Lambda} \omega_{\Lambda^{c}}\right) e^{-\beta \mathbf{H}_{\Lambda}^{\Phi}(\sigma \mid \omega)} \rho_{\Lambda}\left(d \sigma_{\Lambda}\right)
$$

\footnotetext{
${ }^{5}$ See also a general discussion about Banach spaces of interactions in [36].
} 
Theorem 3.23 (Gibbs specification). Let $\Phi$ be a UAC potential and $\beta>0$. The family of kernels $\gamma^{\beta \Phi}=\left(\gamma_{\Lambda}^{\beta \Phi}\right)_{\Lambda \in \mathcal{S}}$ is a specification, called Gibbs specification with (UAC) potential $\Phi$, at inverse temperature $\beta>0$.

Proof: It is straightforward to prove that for a UAC potential, the Hamiltonian with boundary condition is bounded, and thus the partition function exists as a function of the boundary condition. Define now for all $A \in \mathcal{F}, \Lambda \in \mathcal{S}$ and $\sigma \in \Omega$, the density-type function

$$
f_{\Lambda}(\sigma)=\frac{1}{\mathbf{Z}_{\Lambda}^{\beta \Phi}(\sigma)} \cdot e^{-\beta \mathbf{H}_{\Lambda}^{\Phi}(\sigma)} .
$$

There is no boundary condition $\omega$ involved in this function, although it incorporates the partition function. This being $\mathcal{F}_{\Lambda^{c}}$-measurable, one nevertheless recovers

$$
\forall \sigma, \omega \in \Omega, f_{\Lambda}\left(\sigma_{\Lambda} \omega_{\Lambda^{c}}\right)=\frac{1}{Z_{\Lambda}^{\beta \Phi}(\omega)} \cdot e^{-\beta \mathbf{H}_{\Lambda}^{\Phi}(\sigma \mid \omega)} .
$$

We have also $0<\left|f_{\Lambda}\right| \leq 1$ and for all $A \in \mathcal{F}$ and $\omega \in \Omega$,

$$
\gamma_{\Lambda}^{\beta \Phi}(A \mid \omega)=\int_{A} f_{\Lambda}(\sigma) \kappa_{\Lambda}^{\omega}(d \sigma)=\int_{\Omega_{\Lambda}} \mathbf{1}_{A}\left(\sigma_{\Lambda} \omega_{\Lambda^{c}}\right) \cdot f_{\Lambda}\left(\sigma_{\Lambda} \omega_{\Lambda^{c}}\right) \rho_{\Lambda}\left(d \sigma_{\Lambda}\right) .
$$

and it is straightforward to check that $\gamma_{\Lambda}^{\beta \Phi}$ is a probability kernel satisfying properties 1. in Definition 2.21 of a specification. Properness is also directly verified: Let $B \in \mathcal{F}_{\Lambda^{c}} . \forall \sigma, \omega \in \Omega, \mathbf{1}_{B}\left(\sigma_{\Lambda} \omega_{\Lambda^{c}}\right)$ is independent of $\sigma$ and $\mathbf{1}_{B}\left(\sigma_{\Lambda} \omega_{\Lambda^{c}}\right)=\mathbf{1}_{B}\left(\omega_{\Lambda} \omega_{\Lambda^{c}}\right)=\mathbf{1}_{B}(\omega)$. Therefore, for all $\omega \in \Omega$ and $B \in \mathcal{F}_{\Lambda^{c}}$,

$$
\begin{aligned}
& \gamma_{\Lambda}^{\beta \Phi}(B \mid \omega)=\frac{1}{\mathbf{Z}_{\Lambda}^{\beta \Phi}(\omega)} \int_{\Omega_{\Lambda}} \mathbf{1}_{B}\left(\sigma_{\Lambda} \omega_{\Lambda^{c}}\right) e^{-\beta \mathbf{H}_{\Lambda}^{\Phi}(\sigma \mid \omega)} \rho_{\Lambda}\left(d \sigma_{\Lambda}\right) \\
= & \frac{1}{\mathbf{Z}_{\Lambda}^{\beta \Phi}(\omega)} \int_{\Omega_{\Lambda}} \mathbf{1}_{B}\left(\omega_{\Lambda} \omega_{\Lambda^{c}}\right) e^{-\beta \mathbf{H}_{\Lambda}^{\Phi}(\sigma \mid \omega)} \rho_{\Lambda}\left(d \sigma_{\Lambda}\right) \\
= & \frac{\mathbf{1}_{B}(\omega)}{\mathbf{Z}_{\Lambda}^{\beta \Phi}(\omega)} \int_{\Omega_{\Lambda}} e^{-\beta \mathbf{H}_{\Lambda}^{\Phi}(\sigma \mid \omega)} \rho_{\Lambda}\left(d \sigma_{\Lambda}\right)=\mathbf{1}_{B}(\omega) .
\end{aligned}
$$

To prove consistency (2.22), we assume without any loss of generality that $\beta=1$ and consider $\Lambda \subset \Lambda^{\prime} \in \mathcal{S}, A \in \mathcal{F}$ and $\omega \in \Omega$. To prove that $\gamma_{\Lambda^{\prime}}(A \mid \omega)=\gamma_{\Lambda^{\prime}} \gamma_{\Lambda}(A \mid \omega)$, we write

$$
\begin{gathered}
\gamma_{\Lambda^{\prime}}(A \mid \omega)=\int_{\Omega_{\Lambda^{\prime}}} \mathbf{1}_{A}\left(\tau_{\Lambda^{\prime}} \omega_{\Lambda^{\prime c}}\right) f_{\Lambda^{\prime}}\left(\tau_{\Lambda^{\prime}} \omega_{\Lambda^{\prime c}}\right) \rho_{\Lambda^{\prime}}\left(d \tau_{\Lambda^{\prime}}\right) \\
\text { and } \gamma_{\Lambda^{\prime}} \gamma_{\Lambda}(A \mid \omega)=\int_{\Omega} \gamma_{\Lambda}(A \mid \tau) \gamma_{\Lambda^{\prime}}(d \tau \mid \omega) \\
=\int_{\Omega_{\Lambda^{\prime}}}\left(\int_{\Omega_{\Lambda}} \mathbf{1}_{A}\left(\sigma_{\Lambda} \tau_{\Lambda^{\prime} \backslash \Lambda} \omega_{\Lambda^{\prime c}}\right) f_{\Lambda}\left(\sigma_{\Lambda} \tau_{\Lambda^{\prime} \backslash \Lambda} \omega_{\Lambda^{\prime c}}\right) d \sigma_{\Lambda}\right) \cdot f_{\Lambda^{\prime}}\left(\tau_{\Lambda^{\prime}} \omega_{\Lambda^{\prime c}}\right) d \tau_{\Lambda^{\prime}}
\end{gathered}
$$


where we have written $d \sigma_{\Lambda}$ instead of $\rho_{\Lambda}\left(d \sigma_{\Lambda}\right)$. To prove that the family is invariant under expectations w.r.t. the conditioning in intermediate regions, we modify the latter expression in order to extract what is really needed in terms of the functions $f_{\Lambda}$. Write

$$
\gamma_{\Lambda^{\prime}} \gamma_{\Lambda}(A \mid \omega)=\int_{\Omega_{\Lambda^{\prime} \backslash \Lambda}} g_{\Lambda, \Lambda^{\prime}}\left(\tau_{\Lambda^{\prime} \backslash \Lambda}\right) d \tau_{\Lambda^{\prime} \backslash \Lambda}
$$

where, using Fubini's theorem and trivial changes of variables, one can rewrite $g_{\Lambda, \Lambda^{\prime}}\left(\tau_{\Lambda^{\prime} \backslash \Lambda}\right)$ as

$$
\begin{aligned}
& \int_{\Omega_{\Lambda}} f_{\Lambda^{\prime}}\left(\tau_{\Lambda} \tau_{\Lambda^{\prime} \backslash \Lambda} \omega_{\Lambda^{\prime c}}\right) \cdot\left(\int_{\Omega_{\Lambda}} \mathbf{1}_{A}\left(\sigma_{\Lambda} \tau_{\Lambda^{\prime} \backslash \Lambda} \omega_{\Lambda^{\prime c}}\right) f_{\Lambda}\left(\sigma_{\Lambda} \tau_{\Lambda^{\prime} \backslash \Lambda} \omega_{\Lambda^{\prime c}}\right) d \sigma_{\Lambda}\right) d \tau_{\Lambda} \\
& =\int_{\Omega_{\Lambda}} \mathbf{1}_{A}\left(\tau_{\Lambda} \tau_{\Lambda^{\prime} \backslash \Lambda} \omega_{\Lambda^{\prime c}}\right) \cdot f_{\Lambda}\left(\tau_{\Lambda^{\prime}} \omega_{\Lambda^{\prime c}}\right) \cdot\left(\int_{\Omega_{\Lambda}} f_{\Lambda^{\prime}}\left(\sigma_{\Lambda} \tau_{\Lambda^{\prime} \backslash \Lambda} \omega_{\Lambda^{\prime c}}\right) d \sigma_{\Lambda}\right) d \tau_{\Lambda}
\end{aligned}
$$

To get consistency at the level of the density functions $\left(f_{\Lambda}\right)_{\Lambda \in \mathcal{S}}$, one would like to get rid of the last integral in this expression. This is provided by the following lemma, which indicates under which conditions on the family $\left(f_{\Lambda}\right)_{\Lambda \in \mathcal{S}}$ of densities one recovers consistency at the level of specifications. This lemma is in fact crucial to get consistency for the Gibbs kernels ${ }^{6}$.

Lemma 3.27 (Consistency for densities). Let $\left(f_{\Lambda}\right)_{\Lambda \in \mathcal{S}}$ be a family of (strictly) positive measurable functions $f_{\Lambda}$ such that $\forall \Lambda \in \mathcal{S}, \forall \omega \in \Omega$, $\int_{\Omega_{\Lambda}} f_{\Lambda}\left(\sigma_{\Lambda} \omega_{\Lambda^{c}}\right) \rho_{\Lambda}\left(d \sigma_{\Lambda}\right)=1$. The following statements are equivalent:

1. $\forall \Lambda \subset \Lambda^{\prime} \in \mathcal{S}, \forall \omega, \omega^{\prime} \in \Omega$ s.t. $\omega_{\Lambda^{c}}=\omega_{\Lambda^{c}}^{\prime}$,

$$
\frac{f_{\Lambda^{\prime}}\left(\omega^{\prime}\right)}{f_{\Lambda^{\prime}}(\omega)}=\frac{f_{\Lambda}\left(\omega^{\prime}\right)}{f_{\Lambda}(\omega)} .
$$

2. $\forall \Lambda \subset \Lambda^{\prime} \in \mathcal{S}, \forall \omega \in \Omega$,

$$
f_{\Lambda^{\prime}}(\omega)=f_{\Lambda}(\omega) \cdot \int_{\Omega_{\Lambda}} f_{\Lambda^{\prime}}\left(\sigma_{\Lambda} \omega_{\Lambda^{c}}\right) \rho_{\Lambda}\left(d \sigma_{\Lambda}\right) .
$$

Proof: Let us prove that 1 . $\Longrightarrow 2$, writing $d \sigma_{\Lambda}$ instead of $\rho_{\Lambda}\left(d \sigma_{\Lambda}\right)$. Assume (3.28) holds for $\Lambda \subset \Lambda^{\prime} \in \mathcal{S}$ and let $\omega \in \Omega$. Then

$$
\begin{aligned}
f_{\Lambda}(\omega) \cdot \int_{\Omega_{\Lambda}} f_{\Lambda^{\prime}}\left(\sigma_{\Lambda} \omega_{\Lambda^{c}}\right) d \sigma_{\Lambda} & =\int_{\Omega_{\Lambda}} f_{\Lambda}(\omega) f_{\Lambda^{\prime}}\left(\sigma_{\Lambda} \omega_{\Lambda^{c}}\right) d \sigma_{\Lambda} \\
& =\int_{\Omega_{\Lambda}} f_{\Lambda^{\prime}}(\omega) f_{\Lambda}\left(\sigma_{\Lambda} \omega_{\Lambda^{c}}\right) d \sigma_{\Lambda} \\
& =f_{\Lambda^{\prime}}(\omega) \cdot \int_{\Omega_{\Lambda}} f_{\Lambda}\left(\sigma_{\Lambda} \omega_{\Lambda^{c}}\right) d \sigma_{\Lambda}=f_{\Lambda^{\prime}}(\omega)
\end{aligned}
$$

\footnotetext{
${ }^{6}$ This property of specifications will be very useful to play on the conditioning for boundary conditions that coincide outside some finite sets, in particular to get the Kozlov's potential in next section. It corresponds to the key bar-displacement property of [43], where densities of specifications are explicitly introduced.
} 
because $\int_{\Omega_{\Lambda}} f_{\Lambda}\left(\sigma_{\Lambda} \xi_{\Lambda^{c}}\right) d \sigma_{\Lambda}=1$. Thus $1 . \Longrightarrow 2$.

Consider now $\omega, \omega^{\prime}, \Lambda, \Lambda^{\prime}$ as above, with $\omega_{\Lambda^{c}}=\omega_{\Lambda^{c}}^{\prime}$. Using

$f_{\Lambda^{\prime}}(\omega)=f_{\Lambda}(\omega) \cdot \int_{\Omega_{\Lambda}} f_{\Lambda^{\prime}}\left(\sigma_{\Lambda} \omega_{\Lambda^{c}}\right) d \sigma_{\Lambda}, f_{\Lambda^{\prime}}\left(\omega^{\prime}\right)=f_{\Lambda}\left(\omega^{\prime}\right) \cdot \int_{\Omega_{\Lambda}} f_{\Lambda^{\prime}}\left(\sigma_{\Lambda} \omega_{\Lambda^{c}}^{\prime}\right) d \sigma_{\Lambda}$ with $\omega_{\Lambda^{c}}=\omega_{\Lambda^{c}}^{\prime}$, we get

$$
\int_{\Omega_{\Lambda}} f_{\Lambda^{\prime}}\left(\sigma_{\Lambda} \omega_{\Lambda^{c}}\right) d \sigma_{\Lambda}=\int_{\Omega_{\Lambda}} f_{\Lambda^{\prime}}\left(\sigma_{\Lambda} \omega_{\Lambda^{c}}^{\prime}\right) d \sigma_{\Lambda}
$$

and then

$f_{\Lambda^{\prime}}\left(\omega^{\prime}\right) \cdot f_{\Lambda}(\omega) \cdot \int_{\Omega_{\Lambda}} f_{\Lambda^{\prime}}\left(\sigma_{\Lambda} \omega_{\Lambda^{c}}\right) d \sigma_{\Lambda}=f_{\Lambda}\left(\omega^{\prime}\right) \cdot\left(\int_{\Omega_{\Lambda}} f_{\Lambda^{\prime}}\left(\sigma_{\Lambda} \omega_{\Lambda^{c}}\right) d \sigma_{\Lambda}\right) \cdot f_{\Lambda^{\prime}}(\omega)$ and we conclude the proof of the lemma by non-nullness of our Gibbs weights.

To prove Theorem 3.23, we check now that item 1 . is true when $f_{\Lambda}$ is given by (3.24). Consider $\Lambda \subset \Lambda^{\prime} \in \mathcal{S}, \omega$ and $\omega^{\prime}$ s.t. $\omega_{\Lambda^{c}}=\omega_{\Lambda^{c}}^{\prime}$. By definition

$$
\frac{f_{\Lambda^{\prime}}\left(\omega^{\prime}\right)}{f_{\Lambda^{\prime}}(\omega)}=\left(\frac{\mathbf{Z}_{\Lambda^{\prime}}\left(\omega^{\prime}\right)}{\mathbf{Z}_{\Lambda^{\prime}}(\omega)}\right)^{-1} \cdot \frac{\exp \left(-\sum_{A \cap \Lambda^{\prime} \neq \emptyset} \Phi_{A}\left(\omega^{\prime}\right)\right)}{\exp \left(-\sum_{A \cap \Lambda^{\prime} \neq \emptyset} \Phi_{A}(\omega)\right)}
$$

But, by $\mathcal{F}_{A^{-}}$-measurability of $\Phi_{A}$, for $A \subset \Lambda, \omega_{\Lambda^{c}}^{\prime}=\omega_{\Lambda^{c}}$ implies $\Phi_{A}(\omega)=$ $\Phi_{A}\left(\omega^{\prime}\right)$, and thus

$$
\begin{aligned}
\frac{\exp \left(-\sum_{A \cap \Lambda^{\prime} \neq \emptyset} \Phi_{A}\left(\omega^{\prime}\right)\right)}{\exp \left(-\sum_{A \cap \Lambda^{\prime} \neq \emptyset} \Phi_{A}(\omega)\right)} & =e^{-\sum_{A \cap \Lambda^{\prime} \neq \emptyset}\left(\Phi_{A}\left(\omega^{\prime}\right)-\Phi_{A}(\omega)\right)} \\
& =e^{-\sum_{A \cap \Lambda \neq \emptyset}\left(\Phi_{A}\left(\omega^{\prime}\right)-\Phi_{A}(\omega)\right)} .
\end{aligned}
$$

The ratio of the partition functions is also the same for such $\omega$ 's and $\omega^{\prime \prime}$ s and eventually (3.28) holds. This implies Item 2. of the lemma: For all $\Lambda \subset \Lambda^{\prime} \in \mathcal{S}$, for all $\tau, \omega \in \Omega$,

$$
f_{\Lambda}\left(\tau_{\Lambda^{\prime}} \omega_{\Lambda^{\prime c}}\right) \cdot\left(\int_{\Omega_{\Lambda}} f_{\Lambda^{\prime}}\left(\sigma_{\Lambda} \tau_{\Lambda^{\prime} \backslash \Lambda} \omega_{\Lambda^{\prime c}}\right) d \sigma_{\Lambda}\right)=f_{\Lambda^{\prime}}\left(\tau_{\Lambda^{\prime}} \omega_{\Lambda^{\prime c}}\right)
$$

and thus (3.26) holds with

$$
g_{\Lambda, \Lambda^{\prime}}\left(\tau_{\Lambda^{\prime} \backslash \Lambda}\right)=\int_{\Omega_{\Lambda}} \mathbf{1}_{A}\left(\tau_{\Lambda^{\prime}} \omega_{\Lambda^{\prime c}}\right) \cdot f_{\Lambda^{\prime}}\left(\tau_{\Lambda^{\prime}} \omega_{\Lambda^{\prime c}}\right) d \tau_{\Lambda}
$$

yielding consistency

$$
\begin{aligned}
\gamma_{\Lambda^{\prime}} \gamma_{\Lambda}(A \mid \omega) & =\int_{\Omega_{\Lambda^{\prime} \backslash \Lambda}}\left(\int_{\Omega_{\Lambda}} \mathbf{1}_{A}\left(\tau_{\Lambda^{\prime}} \omega_{\Lambda^{\prime c}}\right) \cdot f_{\Lambda^{\prime}}\left(\tau_{\Lambda^{\prime}} \omega_{\Lambda^{\prime c}}\right) d \tau_{\Lambda}\right) d \tau_{\Lambda^{\prime} \backslash \Lambda} \\
& =\int_{\Omega_{\Lambda^{\prime}}} \mathbf{1}_{A}\left(\tau_{\Lambda^{\prime}} \omega_{\Lambda^{\prime c}}\right) f_{\Lambda^{\prime}}\left(\tau_{\Lambda^{\prime}} \omega_{\Lambda^{\prime c}}\right) d \tau_{\Lambda^{\prime}}=\gamma_{\Lambda^{\prime}}(A \mid \omega) .
\end{aligned}
$$


Thus $\gamma^{\beta \Phi}$ is indeed a specification.

The relationships between potentials and Gibbs specifications is not oneto-one: local changes in the potentials can be made without affecting the kernels $\gamma^{\beta \Phi}$, yielding equivalent descriptions of measures. This leads to the concept of physical equivalence ${ }^{7}$.

Definition 3.30 (Physical equivalence). Two potentials $\Phi$ and $\Phi^{\prime}$ are physically equivalent if the Gibbs kernels $\gamma_{\Lambda}^{\beta \Phi}$ and $\gamma_{\Lambda}^{\beta \Phi^{\prime}}$ are the same for all $\Lambda \in \mathcal{S}$.

In particular, physically equivalent potentials define the same Gibbs measure(s), introduced now.

Definition 3.31 (Gibbs measures). A probability measure $\mu \in \mathcal{M}_{1}^{+}(\Omega)$ is said to be a Gibbs measure if there exists a UAC potential $\Phi$ and $\beta>0$ such that $\mu \in \mathcal{G}\left(\gamma^{\beta \Phi}\right)$. We often say that $\mu$ is a Gibbs measure for the $U A C$ potential $\Phi$.

\section{Examples of Gibbs measures and phase transitions:}

\section{One-dimensional homogeneous Ising models}

(a) Ferromagnetic n.n.: We have already told in the previous chapter how ergodic Markov chains could be described as Gibbs measures for the homogeneous Ising model. The converse is also possible, and is indeed achieved in a general framework via the introduction of stochastic matrices defined in terms of the n.n. potential. The one-dimensional n.n. Ising model with coupling $J>0$ and external magnetic field $h$ is described and analyzed in this way in [52] using a matricial formalism that leads in particular to the well known absence of phase transitions in one dimension. When $h>0$, uniqueness is proved with a Gibbs measures $\mu_{\beta}^{h}$ of positive magnetization, that converges weakly when the temperature goes to zero, to the Dirac measure at the all + configuration $\left(\delta_{+}\right)$, while opposite measures $\mu_{\beta}^{-}$and $\delta_{-}$are reached when $h<0$. In absence of magnetic field $h=0$, there is also, in one dimension, a unique (neutral) Gibbs measure $\mu_{\beta}$ but it weakly converges to the convex combination $\frac{1}{2} \delta_{+}+\frac{1}{2} \delta_{-}$, exhibiting then a so called asymptotic loss of tail-triviality responsible of the phase transition observed at low temperature in dimension 2 .

(b) Anti-ferromagnetic n.n.: The same matricial formalism is also used to deal with the anti-ferromagnetic case $J<0$. At high

\footnotetext{
${ }^{7}$ Other equivalence classes and spaces of potentials exist, see [36, 64]. In particular, to get the following equivalence it is crucial to focus on UAC potentials.
} 
magnetic field $|h|>2$, the +-phase is the unique one and weakly converges, when the temperature goes to zero, to $\delta_{+}$. The boundary case $|h|=2$ also leads to uniqueness, leading to a unique phase $\mu_{F}$ described in terms of Fibonacci's numbers reflecting a highly non-trivial phenomenon: As claimed in [52], in spite of the existence of infinitely many ground states ${ }^{8}$ there is no asymptotic loss of tail triviality. This loss occurs when the magnetic field is lower, $|h|<2$, where one gets as a unique Gibbs measure a convex combination $\mu=\frac{1}{2} \mu_{\beta}^{ \pm}+\frac{1}{2} \mu^{\mp}$ of two symmetric measures whose typical configurations have either mostly pluses on a the (say) odd sublattice and mostly minuses on the even one. When the temperature goes to zero, $\mu$ weakly converges to a similar convex combination of Dirac measures $\frac{1}{2} \delta_{ \pm}+\frac{1}{2} \delta_{\mp}$, see again a detailed analysis in [52].

(c) Long-range one dimensional Ising models: The potential has already been introduced in the beginning of this section. When the polynomial decay $r=1$, it is not UAC, but a formalism that corresponds to so-called Coulomb interactions can be developed within the weaker notion of uniform convergence, see [113] and also the previous chapter. For $r>1$, this has been studied by e.g. $[32,114]$ and it leads in particular to phase transitions in one dimension when $1<r<2$. Phase transition also occurs in the case $r=2$, yielding a particular decay of correlations known as a Thouless effect, see [49].

2. 3d Ising models: We shall be laconic to describe this very important example of mathematical statistical mechanics: Theorem 2.27 is not valid in dimension $d \geq 3$ and they do exist non-translationinvariant extreme Gibbs measures. This has been achieved by Dobrushin in [28], with a shorter proof in [2], and these non-translationinvariant so-called Dobrushin states are related to the stability of an interface between a +-like phase and a --like one, and to each interface corresponds an extremal Gibbs measure, in addition to the usual +- and --phases. This example is very relevant for comparing the notions of ergodic and extremal Gibbs measures discussed at the end of the previous chapter.

3. Ising models on Cayley trees: This example is also very interesting from the ergodic vs. extreme point of view and there exists as well an (uncountable) infinite number of extremal Gibbs measures at low temperature, and depending on the temperature there could

\footnotetext{
${ }^{8}$ Ground states are the minimizers of the Hamiltonian used to describe the phases at zero temperature and by extension to low temperatures within the Pirogov-Sinai theory [106].
} 
exist two or three translation-invariant ones, see all the work done in $[8,57,62,107]$ and a whole chapter in [52].

4. Kac-models: A careful adaptation of the Peierls argument allows to establish the occurrence of a phase transition for this model at low temperature and long enough range, see $[12,18]$ in dimension $d \geq 2$.

Theorem 3.32. For $d \geq 2$, for any $\beta>1$, there exists $\gamma=\gamma(\beta)$ such that for all $\gamma<\gamma(\beta)$, there exists at least two distinct $D L R$ measures $\mu_{\gamma}^{-} \neq \mu_{\gamma}^{+}$.

Now that our central objects are properly defined, we can prove a previous claim providing Gibbs measures as the main example of quasilocal measures. It is the easiest part of the link between these two notions, a partial converse statement will be established in next section.

Theorem 3.33. Let $\beta>0$ and $\Phi$ be a UAC potential. Then the Gibbs specification $\gamma^{\beta \Phi}$ is quasilocal. Thus, any Gibbs measure is also quasilocal.

Proof: If $\Phi$ be UAC potential, it implies in particular that:

$$
\sum_{A \in \mathcal{S}, A \cap \Lambda \neq \emptyset} \sup _{\omega \in \Omega}\left|\Phi_{A}(\omega)\right|<+\infty
$$

which in turns implies that for all $\Lambda \in \mathcal{S}, H_{\Lambda}^{\Phi}$ is a quasilocal function. Indeed, if $\mathcal{S} \ni \Lambda^{\prime} \supset \Lambda$ and consider two configurations $\sigma$ and $\omega$ such that $\sigma_{\Lambda^{\prime}}=\omega_{\Lambda^{\prime}}$, we have

$$
\left|\mathbf{H}_{\Lambda}^{\Phi}(\omega)-\mathbf{H}_{\Lambda}^{\Phi}(\sigma)\right| \leq 2 \sum_{A \in \mathcal{S}, A \cap \Lambda \neq \emptyset, A \cap \Lambda^{\prime c} \neq \emptyset} \sup _{\omega \in \Omega}\left|\Phi_{A}(\omega)\right|
$$

and the latter converges to zero as a consequence of (3.34). Thus, one gets the quasilocality of the Hamiltonians:

$$
\lim _{\Lambda^{\prime} \uparrow \mathcal{S}} \sup _{\sigma, \omega \in \Omega, \sigma_{\Lambda^{\prime}}=\omega_{\Lambda^{\prime}}}\left|\mathbf{H}_{\Lambda}^{\Phi}(\omega)-\mathbf{H}_{\Lambda}^{\Phi}(\sigma)\right|=0 .
$$

Quasilocality of Gibbs specifications follows.

Remark 3.35 (Uniform convergence and quasilocality). Requiring for a potential to be uniformly absolutely convergent is actually too strong a requirement for merely proving the quasilocality of the Gibbs specification. Uniform convergence is actually enough to prove the quasilocality of the Hamiltonian. In such a case, one has

$$
\sup _{\sigma, \omega \in \Omega, \sigma_{\Lambda^{\prime}}=\omega_{\Lambda^{\prime}}}\left|\mathbf{H}_{\Lambda}^{\Phi}(\omega)-\mathbf{H}_{\Lambda}^{\Phi}(\sigma)\right| \leq 2 \sup _{\omega \in \Omega}\left|\sum_{A \in \mathcal{S}, A \cap \Lambda \neq \emptyset, A \cap \Lambda^{\prime c} \neq \emptyset} \Phi_{A}(\omega)\right|
$$


and

$$
\lim _{\Lambda^{\prime} \uparrow \mathcal{S}} \sup _{\sigma, \omega \in \Omega}\left|\sum_{A \in \mathcal{S}, A \cap \Lambda \neq \emptyset, A \cap \Lambda^{\prime c} \neq \emptyset} \Phi_{A}(\omega)\right|=0
$$

means the uniform convergence of this potential. Thus, when the potential is uniformly convergent, the Hamiltonian is a well-defined quasilocal function and so is the specification.

Remark 3.36 (Non-Gibbsianness and essential discontinuity). Let $\mu$ be a Gibbs measure: By Theorem 3.33, there exists a quasilocal specification $\gamma$ s.t. $\mu \in \mathcal{G}(\gamma)$ and

$$
\forall A \in \mathcal{F}, \mu\left[A \mid \mathcal{F}_{\Lambda^{c}}\right](\cdot)=\mathbb{E}_{\mu}\left[\mathbf{1}_{A} \mid \mathcal{F}_{\Lambda^{c}}\right](\cdot)=\gamma_{\Lambda}(A \mid \cdot) \mu \text {-a.s. }
$$

so that there exists always one continuous version, as a function of the boundary condition $\omega$, of the conditional probabilities of $\mu$ with respect to the $\sigma$-algebra generated by the outside of finite sets. This will be used in Chapter 5 to detect non-Gibbsianness by proving the existence of special configurations that are point of essential discontinuities, for which there exists conditional expectations of local functions that have no continuous version.

A Gibbs specification is quasilocal but the converse is not true in general. However, most of the quasilocal specifications are Gibbsian, as we prove in the sequel.

\subsubsection{Gibbs representation theorem}

In this section, we want to characterize a Gibbs measure at the level of specifications: Let $\mu$ be a DLR measure, i.e. such that there is a specification $\gamma$ with $\mu \in \mathcal{G}(\gamma)$. To characterize $\mu$ as a Gibbs measure, one should manage to express the weights of configurations in an exponential form and in some sense every configuration should receive a non-zero weight. One says that the specification has to be non-null in the following sense:

Definition 3.37 (Uniform non-nullness). A specification $\gamma$ is said to be uniformly non-null iff $\forall \Lambda \in \mathcal{S}, \exists \alpha_{\Lambda}, \beta_{\Lambda}$ with $0<\alpha_{\Lambda} \leq \beta_{\Lambda}<\infty$ s.t.

$$
0<\alpha_{\Lambda} \rho(A) \leq \gamma_{\Lambda}(A \mid \omega) \leq \beta_{\Lambda} \rho(A), \forall \omega \in \Omega, \forall A \in \mathcal{F}
$$

Simple non-nullness, in the sense that $\rho(A)>0 \Longrightarrow \gamma_{\Lambda}(A \mid \omega)>0$, for all $\omega \in \Omega$, is equivalent to uniform non-nullness when $\gamma$ is quasilocal, [52]. It is also-called the finite-energy condition in percolation circles. A measure $\mu$ is then said to be (uniformly) non-null if there exists a (uniformly) non-null specification $\gamma$ such that $\mu \in \mathcal{G}(\gamma)$. 
We are now ready to give a partial converse statement of Theorem 3.33 . For the purpose of this theorem, the inverse temperature $\beta$ has been incorporated in the potential.

Theorem 3.39 (Gibbs representation theorem [72, 43]). Let $\mu$ be a quasilocal and uniformly non-null probability measure on $(\Omega, \mathcal{F})$. Then $\mu$ is a Gibbs measure, i.e. there exists a UAC potential $\Psi$ such that $\mu \in \mathcal{G}\left(\gamma^{\Psi}\right)$.

Proof: Let $\mu$ non-null and quasilocal: There exists a quasilocal specification $\gamma$ such that $\mu \in \mathcal{G}(\gamma)$ and (3.38) holds, and let us try to guess which necessary property a potential should have to get $\gamma=\gamma^{\beta \Phi}$, by considering such a Gibbs specification $\gamma$ first. Among all the physically equivalent potentials that define this specification, let us also assume for the moment that a vacuum potential $\Phi^{+}$exists, with a vacuum state denoted by + . The vacuum property and its link with free boundary conditions will be very useful to relate the specification and the potential. Indeed, considering $\Lambda \in \mathcal{S}$, then one obviously has $H_{\Lambda}^{\Phi^{+}}(+\mid+)=0$ by the vacuum property and thus

$$
\gamma_{\Lambda}(+\mid+)=\frac{1}{Z_{\Lambda}(+)}
$$

so for any other configuration $\sigma \in \Omega$

$$
\gamma_{\Lambda}(\sigma \mid+)=\gamma_{\Lambda}(+\mid+) e^{-H_{\Lambda}^{\Phi^{+}}(\sigma \mid+)} .
$$

To exploit consistency via Lemma (3.27), we introduce the density $f_{\Lambda}(\sigma):=$ $\gamma_{\Lambda}(\sigma \mid \sigma)$. Consistency implies that it satisfies the conditions of Lemma 3.27, and we shall use indifferently both expressions, in terms of $f$ or in terms ${ }^{9}$ of $\gamma$. Then, by non-nullness and the defining equation (3.24),

$$
H_{\Lambda}^{\Phi^{+}}(\sigma \mid+)=-\ln \frac{\gamma_{\Lambda}(\sigma \mid+)}{\gamma_{\Lambda}(+\mid+)}=\ln \frac{f_{\Lambda}(+)}{f_{\Lambda}\left(\sigma_{\Lambda}+\Lambda^{c}\right)} .
$$

It then possible to derive a vacuum potential from this Hamiltonian, because prescribing a vacuum state as boundary condition is equivalent to consider the Hamiltonian with free boundary condition, for which the use of an inversion formula from Moebius is direct. Indeed, the vacuum condition yields

$$
H_{\Lambda}^{\Phi^{+}}(\sigma \mid+)=\sum_{A \cap \Lambda \neq \emptyset} \Phi_{A}^{+}\left(\sigma_{\Lambda}+\Lambda^{c}\right)=\sum_{A \subset \Lambda} \Phi_{A}^{+}(\sigma)+\sum_{A \cap \Lambda \neq \emptyset, A \cap \Lambda^{c} \neq \emptyset} \Phi_{A}^{+}\left(+{ }_{\Lambda}+{ }_{\Lambda^{c}}\right)
$$

\footnotetext{
${ }^{9}$ The formulation in terms of the density $f_{\Lambda}$ is handy to use consistency via Lemma 3.27 , while the expression in terms of $\gamma$ is more familiar. Fernández [43] has introduced densities for specifications and has expressed Lemma 3.27 in terms of $\gamma$ directly.
} 
where the last sum is null by the vacuum property. Thus

$$
\left.\forall \Lambda \in \mathcal{S}, \forall \sigma \in \Omega, H_{\Lambda}^{\Phi^{+}}(\sigma \mid+)=H_{\Lambda}^{\Phi^{+}, f}(\sigma):=\sum_{A \subset \Lambda} \Phi_{A}^{+}(\sigma)\right) .
$$

In particular one gets directly the single-site potentials:

$$
\forall i \in S, \forall \sigma \in \Omega, \Phi_{\{i\}}^{+}(\sigma)=H_{\{i\}}^{\Phi^{+}, f}(\sigma)=-\ln \frac{\gamma_{\{i\}}(\sigma \mid+)}{\gamma_{\{i\}}(+\mid+)} .
$$

To get an insight of the mechanism of the Moebius inversion formula, which will enables us to rewrite $\Phi^{+}$from $\gamma$, let us use (3.40) to derive the potential for finite regions consisting of two and three sites, for a fixed $\sigma$ that we forget in the notation. For $\Lambda=\{i, j\}$, write

$$
H_{\{i, j\}}^{\Phi^{+}, f}=\Phi_{\{i\}}^{+}+\Phi_{\{j\}}^{+}+\Phi_{\{i, j\}}^{+}
$$

so, using the single-site expression (3.41), one gets for all $\sigma \in \Omega$

$$
\begin{aligned}
\Phi_{\{i, j\}}^{+} & =H_{\{i, j\}}^{\Phi^{+}, f}-H_{\{i\}}^{\Phi^{+}, f}-H_{\{j\}}^{\Phi^{+}, f} \\
& =-\ln \frac{\gamma_{\{i, j\}}(\sigma \mid+)}{\gamma_{\{i, j\}}(+\mid+)}+\ln \frac{\gamma_{\{i\}}(\sigma \mid+)}{\gamma_{\{i\}}(+\mid+)}+\ln \frac{\gamma_{\{j\}}(\sigma \mid+)}{\gamma_{\{j\}}(+\mid+)} .
\end{aligned}
$$

For $\Lambda=\{i, j, k\}$, write similarly, thanks to the vacuum condition,

$$
\begin{aligned}
H_{\{i, j, k\}}^{\Phi^{+}, f} & =\Phi_{\{i\}}^{+}+\Phi_{\{j\}}^{+}+\Phi_{\{k\}}^{+}+\Phi_{\{i, j\}}^{+}+\Phi_{\{i, k\}}^{+}+\Phi_{\{j, k\}}^{+}+\Phi_{\{i, j, k\}}^{+} \\
& =\Phi_{\{i\}}^{+}+\Phi_{\{j\}}^{+}+\Phi_{\{k\}}^{+}+H_{\{i, j\}}^{\Phi^{+}, f}-H_{\{i\}}^{\Phi^{+}, f}-H_{\{j\}}^{\Phi^{+}, f}+H_{\{i, k\}}^{\Phi^{+}, f} \\
& -H_{\{i\}}^{\Phi^{+}, f}-H_{\{k\}}^{\Phi^{+}, f}+H_{\{j, k\}}^{\Phi^{+}, f}-H_{\{j\}}^{\Phi^{+}, f}-H_{\{k\}}^{\Phi^{+}, f}+\Phi_{\{i, j, k\}}^{+} .
\end{aligned}
$$

So,

$H_{\{i, j, k\}}^{\Phi^{+}, f}=-H_{\{i\}}^{\Phi^{+}, f}-H_{\{j\}}^{\Phi^{+}, f}-H_{\{k\}}^{\Phi^{+}, f}+H_{\{i, j\}}^{\Phi^{+}, f}+H_{\{i, k\}}^{\Phi^{+}, f}+H_{\{j, k\}}^{\Phi^{+}, f}+\Phi_{\{i, j, k\}}^{+}$

and thus

$\Phi_{\{i, j, k\}}^{+}=H_{\{i, j, k\}}^{\Phi^{+}, f}-H_{\{i, j\}}^{\Phi^{+}, f}-H_{\{i, k\}}^{\Phi^{+}, f}-H_{\{j, k\}}^{\Phi^{+}, f}+H_{\{i\}}^{\Phi^{+}, f}+H_{\{j\}}^{\Phi^{+}, f}+H_{\{k\}}^{\Phi^{+}, f}$.

Proceeding by induction, one could reconstruct the potential in this way. It is actually formally proved using the following formula, proved in this way e.g. in [43].

Proposition 3.42 (Moebius "inclusion-exclusion" inversion formula). Let $\mathcal{S}$ be a countable set of finite sets and $H=\left(H_{\Lambda}\right)_{\Lambda \in \mathcal{S}}$ and $\Phi=\left(\Phi_{A}\right)_{A \in \mathcal{S}}$ be set functions from $\mathcal{S}$ to $\mathbb{R}$. Then

$$
\forall \Lambda \in \mathcal{S}, H_{\Lambda}=\sum_{A \subset \Lambda} \Phi_{A} \Longleftrightarrow \forall A \in \mathcal{S}, \Phi_{A}=\sum_{B \subset A}(-1)^{|A \backslash B|} H_{B}
$$


We use it and propose then the

Definition 3.44. A vacuum potential for a given specification $\gamma$ is the potential defined for all $\sigma \in \Omega$ by $\Phi_{\emptyset}^{+}=0$ and for all $A \in \mathcal{S}$,

$$
\Phi_{A}^{+}(\sigma)=-\sum_{B \subset A}(-1)^{|A \backslash B|} \ln \frac{\gamma_{B}(\sigma \mid+)}{\gamma_{B}(+\mid+)}=\sum_{B \subset A}(-1)^{|A \backslash B|} \ln \frac{f_{B}(+)}{f_{B}\left(\sigma_{B}+B^{c}\right)} .
$$

Lemma 3.46 (Convergence and consistency of the vacuum potential). Let $\gamma$ be any quasilocal and non-null specification. Then $\Phi^{+}=$ $\left(\Phi_{A}^{+}\right)_{A \in \mathcal{S}}$ defined by (3.45) is a vacuum potential with vacuum state $+\in \Omega$, for any reference configuration $+\in \Omega$. It is moreover convergent and its corresponding Gibbs specification $\gamma^{\Phi}$ coincides with $\gamma$.

Proof: $\Phi^{+}$is obviously a potential. Consider any reference configuration $+\in \Omega$ and prove first that $\Phi^{+}$satisfies the vacuum condition. It will be crucial to get consistency. Consider $A \in \mathcal{F}$ and $\sigma \in \Omega$ such that there exists $i \in A$ where $\sigma_{i}=+_{i}$ and write

$$
\Phi_{A}^{+}(\sigma)=-\sum_{B \subset A}(-1)^{|A \backslash B|} \ln \frac{\gamma_{B}(\sigma \mid+)}{\gamma_{B}(+\mid+)}=\sum_{B \subset A}(-1)^{|A \backslash B|} H_{B}^{\Phi^{+}, f}(\sigma)
$$

where by the Moebius formula, one has for all $B \in \mathcal{S}$,

$$
H_{B}^{\Phi^{+}, f}(\sigma)=\sum_{A \subset B} \Phi_{A}^{+}(\sigma)=-\ln \frac{\gamma_{B}(\sigma \mid+)}{\gamma_{B}(+\mid+)}=\ln \frac{f_{B}(+)}{f_{\Lambda}\left(B_{\Lambda}+\Lambda^{c}\right)} .
$$

Using Equation (3.28), one first gets

$$
\forall i \in B \subset A, H_{B}^{\Phi^{+}, f}(\sigma)=H_{B \backslash i}^{\Phi^{+}, f}(\sigma) .
$$

Indeed, by consistency property of the specification, one can rewrites

$$
\frac{f_{B}(+)}{f_{B}\left(\sigma_{B}+{ }_{B^{c}}\right)}=\frac{f_{B}\left(+{ }_{B \backslash i}+{ }_{i}\right)}{f_{B}\left(\sigma_{B \backslash i}+{ }_{i}\right)}=\frac{f_{B \backslash i}(+)}{\left.f_{B \backslash i}\left(\sigma_{B \backslash i}+{ }_{i}\right)\right)}
$$

to eventually get (3.49). Now define for any site $i \in S$ a partition of $\mathcal{S}$ by $\mathcal{S}=\mathcal{S}_{A, i} \cup \mathcal{S}_{A, i}^{c}$ with $\mathcal{S}_{A, i}=\{V \in \mathcal{S}, V \subset A, V \ni i\}$. An obvious bijection from $\mathcal{S}_{A, i}$ to $\mathcal{S}_{A, i}^{c}$ links $B \in \mathcal{S}_{A, i}$ to $B \backslash i \in \mathcal{S}_{A, i}^{c}$, so one gets

$$
\begin{aligned}
\Phi_{A}^{+}(\omega) & =\sum_{B \in \mathcal{S}_{A, i}}(-1)^{|A \backslash B|} H_{B}^{\Phi^{+}}(\omega)+\sum_{B \in \mathcal{S}_{A, i}^{c}}(-1)^{|A \backslash B|} H_{B}^{\Phi^{+}}(\omega) \\
& =\sum_{B \in \mathcal{S}_{A, i}}\left[(-1)^{|A \backslash B|} H_{B}^{\Phi^{+}}(\omega)+(-1)^{|A \backslash\{B \backslash i\}|} H_{B \backslash i}^{\Phi^{+}}(\omega)\right] \\
& =\sum_{B \in \mathcal{S}_{A, i}}(-1)^{|A \backslash B|}\left[H_{B}^{\Phi^{+}}(\omega)-H_{B \backslash i}^{\Phi^{+}}(\omega)\right] \\
& =0
\end{aligned}
$$


and $\Phi^{+}$is indeed a vacuum potential associated to the specification $\gamma$. Let us check that in the non-null quasilocal case it is consistent with $\gamma$ and has the required asymptotic properties. We first need to prove that we can always define

$$
\forall \sigma, \omega \in \Omega, H_{\Lambda}^{\Phi^{+}}(\sigma \mid \omega)=\sum_{A \cap \Lambda \neq \emptyset, A \in \mathcal{S}} \Phi_{A}^{+}\left(\sigma_{\Lambda} \omega_{\Lambda^{c}}\right)
$$

in order to extend the definition of the Hamiltonian with free b.c. (3.48) to an Hamiltonian with any $\omega \in \Omega$ as a boundary condition. It amounts to proving the convergence of the potential, i.e. that for all $\sigma \in \Omega$,

$$
H_{\Lambda}^{\Phi^{+}}(\sigma):=\sum_{A \cap \Lambda \neq \emptyset, A \in \mathcal{S}} \Phi_{A}^{+}(\sigma)<+\infty
$$

in the sense that the limit as $\Delta \uparrow \mathcal{S}$ of the net $\left(\sum_{A \cap \Lambda \neq \emptyset, A \subset \Delta} \Phi_{A}^{+}(\sigma)\right)_{\Delta \in \mathcal{S}}$ is finite. Recall that we have already been able to define the Hamiltonian with free b.c. as

$$
H_{\Lambda}^{\Phi^{+}, f}(\sigma)=\sum_{A \subset \Lambda} \Phi_{A}^{+}(\sigma)=\ln \frac{f_{\Lambda}(+)}{f\left(\sigma_{\Lambda}+\Lambda^{c}\right)} .
$$

To prove now that it is a convergent potential using the quasilocality of the function $\omega \longmapsto f_{\Lambda}\left(\sigma_{\Lambda} \omega_{\Lambda^{c}}\right)$, we re-write

$$
\sum_{A \cap \Lambda \neq \emptyset, A \subset \Delta} \Phi_{A}^{+}(\sigma)=\sum_{A \subset \Delta} \Phi_{A}^{+}(\sigma)-\sum_{A \subset \Delta \cap \Lambda^{c}} \Phi_{A}^{+}(\sigma) .
$$

Using twice Moebius inversion formula (3.43), one obtains

$$
\sum_{A \subset \Delta} \Phi_{A}^{+}(\sigma)=\ln \frac{f_{\Delta}(+)}{f_{\Delta}\left(\sigma_{\Delta}+\Delta^{c}\right)}
$$

and

$$
\left.\sum_{A \subset \Delta \cap \Lambda^{c}} \Phi_{A}^{+}(\sigma)=\ln \frac{f_{\Delta \cap \Lambda^{c}}(+)}{f_{\Delta \cap \Lambda^{c}}\left(\sigma_{\Delta \cap \Lambda^{c}}+\Delta^{c} \cup \Lambda\right.}\right) .
$$

By consistency (Lemma 3.27), we get $\ln \frac{f_{\Delta}(+)}{f_{\Delta}\left(\sigma_{\Delta \cap \Lambda^{c}+\Delta^{c} \cup \Lambda}\right)}$ for the second term, because, on $\Delta \cap \Lambda^{c}$, the two involved configurations coincide and eventually

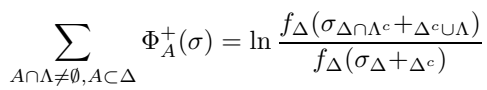

and using again Lemma (3.27) with the configurations $\sigma_{\Delta \cap \Lambda^{c}}+{ }_{\Delta^{c} \cup \Lambda}$ and $\left.\sigma_{\Delta}+\Delta^{c}\right)$ which agree outside $\Delta \cap \Lambda$, we get

$$
\left.\sum_{A \cap \Lambda \neq \emptyset, A \subset \Delta} \Phi_{A}^{+}(\sigma)=\ln \frac{f_{\Lambda \cap \Delta}\left(\sigma_{\Delta \cap \Lambda^{c}}+\Delta^{c} \cup \Lambda\right.}{}\right) .
$$


Let $\Delta \uparrow S$ in the sense defined. For $\Delta \supset \Lambda$, one gets

$$
\sum_{A \cap \Lambda \neq \emptyset, A \subset \Delta} \Phi_{A}^{+}(\sigma)=\ln \frac{f_{\Lambda}\left(+{ }_{\Lambda} \sigma_{\Delta \backslash \Lambda}+\Delta^{c}\right)}{f_{\Lambda}\left(\sigma_{\Delta}+\Delta^{c}\right)} .
$$

Thus quasilocality implies that the potential $\Phi$ is convergent and that for all $\sigma \in \Omega$,

$$
H_{\Lambda}^{\Phi^{+}}(\sigma)=\sum_{A \cap \Lambda \neq \emptyset, A \in \mathcal{S}} \Phi_{A}^{+}(\sigma)=-\ln \frac{\gamma_{\Lambda}(\sigma \mid \sigma)}{\gamma_{\Lambda}(+\mid \sigma)}=\ln \frac{f_{\Lambda}\left(+{ }_{\Lambda} \sigma_{\Lambda^{c}}\right)}{f_{\Lambda}(\sigma)}<+\infty .
$$

Hence, every quasilocal and non-null specification $\gamma$ is consistent with the convergent vacuum potential $\Phi^{+}$whose the Hamiltonian with boundary condition $\omega \in \Omega$ is defined for all $\sigma \in \Omega$

$$
\forall \omega \in \Omega, H_{\Lambda}^{\Phi^{+}}(\sigma \mid \omega)=-\ln \frac{\gamma_{\Lambda}(\sigma \mid \omega)}{\gamma_{\Lambda}(+\mid \omega)}=\ln \frac{f_{\Lambda}\left(+{ }_{\Lambda} \omega_{\Lambda^{c}}\right)}{f_{\Lambda}\left(\sigma_{\Lambda} \omega_{\Lambda^{c}}\right)}<+\infty
$$

This proves Lemma 3.46: Any quasilocal and non-null specification is consistent with a convergent potential.

Unfortunately, this vacuum potential is not UAC in the sense of (3.15). To gain summability and absoluteness, Kozlov [72] introduced a particular re-summation procedure by telescoping the terms of the Hamiltonian with free boundary conditions in large enough annuli to recover absoluteness, but carefully keeping consistency, to eventually get a potential $\Psi$ such that, for all $\sigma \in \Omega$

$$
\sum_{A \subset \Lambda} \Psi_{A}(\sigma)=H_{\Lambda}^{\Phi^{+}, f}(\sigma)=\sum_{A \subset \Lambda} \Phi_{A}^{+}(\sigma)=\ln \frac{f_{\Lambda}(+)}{f\left(\sigma_{\Lambda}+\Lambda^{c}\right)}
$$

holds together with the extra summability property

$$
\forall i \in S, \sum_{A \in \mathcal{S}, A \ni i} \sup _{\omega}\left|\Psi_{A}(\omega)\right|<+\infty
$$

We shall describe it formally following the pedagogical exposition of Fernández [43], and describe a bit more explicitly the telescoping at the end of this proof.

In our settings with a finite single-spin state space, non-nullness and quasilocality can be reduced to site-characterizations that are very useful to get the stronger summability around sites (3.52). Introduce, for any site $i \in S$ and any cube $\Lambda_{n}$, the quantities

$$
m_{i}:=\inf _{\omega} f_{\{i\}}(\omega)=\inf _{\omega \in \Omega} \gamma_{\{i\}}(\omega \mid \omega)
$$


and

$$
g_{i}(n)=\sup _{\omega}\left|f_{\{i\}}\left(\omega_{\Lambda_{n}}+\Lambda_{n}^{c}\right)-f_{\{i\}}(\omega)\right|
$$

By non-nullness, one has $m_{i}>0$ for all $i \in S$ and quasilocality reads

$$
\forall i \in S, g_{i}(n) \underset{n \rightarrow \infty}{\longrightarrow} 0
$$

Starting from the expression of the Hamiltonians (3.47) in terms of the vacuum potential, which itself is expressed as the logarithm of ratios of densities, Kozlov used the inequality

$$
\left|\ln \frac{a}{b}\right| \leq \frac{|a-b|}{\min (\mathrm{a}, \mathrm{b})}, \forall a, b>0
$$

to get that for all $i \in S$

$$
\sup _{\omega}\left|\sum_{A \subset \Lambda_{n}, A \ni i} \Phi^{+}(\sigma)\right| \leq \frac{g_{i}(n)}{m_{i}}
$$

and in particular that

$$
\sup _{\omega}\left|\sum_{A \subset \Lambda_{n}, A \ni i} \Phi^{+}(\sigma)-\sum_{A \subset \Lambda_{n-1}, A \ni i} \Phi^{+}(\sigma)\right| \leq \frac{g_{i}(n)+g_{i}(n-1)}{m_{i}} .
$$

Kozlov used then these bounds to reduce the lack of absolute convergence by grouping terms of the vacuum potential within intermediate annuli chosen large enough to exploit quasilocality. To do so, the telescoping has thus to integrate larger boxes, i.e. has to be performed along subsequences of cubes $\Lambda_{n_{k}^{i}}, k \geq 1$ in (3.54) chosen such that for any $i \in S$,

$$
\sum_{k \geq 1} g_{i}\left(n_{k}^{i}\right)<\infty
$$

which is always possible because for any $i \in S$, the sequence $\left(g_{i}(n)\right)_{n \in \mathbb{N}}$ converges to zero.

For any $i \in S$, we consider then the subsequence of cubes $\Lambda_{n_{k}^{i}}$, centered in $i$, of radius $n_{k}^{i}$ such that the annuli $\Lambda_{n_{k}^{i}} \backslash \Lambda_{n_{k-1}^{i}}$ is large enough, as we shall see. This size will allow the use of the bounds (3.53) for any $i \in S$ and to get the right summability properties, the telescoping is done by following the bonds along these cubes, adding at each steps the terms of the vacuum potential that correspond to bonds of the annulus, and that were not in the previous cubes. Define then, for any $i \in S$, any $k \geq 1$

$$
S_{k}^{i}=\left\{B \subset \Lambda_{n_{k}}^{i}: B \ni i\right\} \backslash S_{k-1}^{i}
$$


with $S_{0}^{i}=\{i\}$, and introduce the potential ${ }^{10} \tilde{\Psi}$ defined by

$\tilde{\Psi}_{A}(\sigma)=\left\{\begin{array}{l}\sum_{B \in S_{k}} \Phi_{B}^{+}(\sigma) \quad \text { if } \quad A=\Lambda_{n_{k}}^{i} \text { for some } k \geq 1, \text { some } i \in S . \\ 0 \quad \text { otherwise. }\end{array}\right.$

By (3.53), one has

$$
\begin{aligned}
\sup _{\omega}\left|\tilde{\Psi}_{A}(\sigma)\right| & =\sup _{\omega}\left|\sum_{B \ni i, B \subset \Lambda_{n_{k}^{i}}} \tilde{\Phi}_{B}^{+}(\sigma)-\sum_{B \ni i, B \subset \Lambda_{n_{k-1}^{i}}} \tilde{\Phi}_{B}^{+}(\sigma)\right| \\
& \leq \frac{g_{i}\left(n_{k}^{i}\right)+g_{i}\left(n_{k-1}^{i}\right)}{m_{i}} .
\end{aligned}
$$

in such a way that one has the right summability property at the site $i$ :

$$
\sum_{A \ni i} \sup _{\omega}\left|\tilde{\Psi}_{A}^{i}(\sigma)\right| \leq \frac{2}{m_{i}} \cdot \sum_{k \geq 1} g_{i}\left(n_{k}^{i}\right)<+\infty .
$$

Nevertheless, we need to do the telescoping more carefully to keep the consistency, in general lost in the procedure above: For a given $B \in \mathcal{S}$, the same vacuum interaction $\Phi_{B}$ could have been used more than once. To avoid it, one has to find a way of grouping terms of the vacuum interaction without using the terms already used, i.e. one has to run the sequence of cubes by using any finite set $B$ of bonds only once. To do so, Fernández [43] proposed the following presentation of Kozlov's potential, now denoted by $\Psi$. The sites of the lattice are now lexicographically ordered and still generically denoted by $i$. For any site, one replaces the previous subsequence of cubes $\Lambda_{n_{k}^{i}}$ by rectangles around it that do not incorporate $B$ 's (or i's) already considered. Hence, one defines for each $i=1,2, \ldots$, a sequence $\left(L_{k}^{i}\right)_{k \geq 1}$ defined for all $i, k \geq 1$ by

$$
L_{k}^{i}=\left\{j \in S: i \leq j \leq r_{k}^{i}\right\}
$$

where the diameters $r_{k}^{i}$ are chosen such that $n_{k}^{i}=\operatorname{diam}\left(L_{k}^{i}\right)=r_{k}^{i}-i$ in order to keep the same large enough sequence of annuli. These groups of bonds will be the only ones involved in the potential and to perform a correct re-summation procedure, one defines for any site $i \in S$, a family of disjoints subsets of $\mathcal{S}$ containing $i$ by $S_{0}^{i}=\{i\}$ and

$$
S_{k}^{i}=\left\{B \subset L_{k}^{i}: B \ni i\right\} \backslash S_{k-1}^{i}
$$

\footnotetext{
${ }^{10}$ This potential is not yet the Kozlov potential, so we write it $\tilde{\Psi}$, because the resummation uses several times terms involving sites $i$.
} 
in such a way that $B \in \cup_{j=1}^{i} \cup_{k \geq 1} S_{j}^{i}$ and any $B$ containing $i$ is uniquely contained in one of them. By this procedure, any set of bonds B is considered only once and we get the Kozlov potential $\Psi$ defined by

$$
\Psi_{A}(\sigma)= \begin{cases}\sum_{B \in S_{k}^{i}} \Phi_{B}^{+}(\sigma) & \text { if } A=L_{k}^{i} \text { for some }(i, k), i \in S, k \geq 1 \\ 0 & \text { otherwise. }\end{cases}
$$

yielding the following

Lemma 3.56. The Kozlov's potential $\Psi$ defined by (3.55) is a UAC potential consistent with the non-null and quasilocal specification $\gamma$, and thus any quasilocal measure $\mu \in \mathcal{G}(\gamma)$ is a Gibbs measure.

Consistency holds because the careful procedure yields the same Hamiltonian with free boundary conditions for the vacuum and Kozlov potentials, and the required convergence, valid for all $i \in S$, is due to the choice of the subsequences $n_{k}^{i}$ :

$$
\sum_{A \in \mathcal{S}, A \ni i} \sup _{\omega}\left|\Psi_{A}(\omega)\right| \leq \sum_{j=1}^{i} \sum_{k \geq 1} \sup _{\omega}\left|\Psi_{L_{k}^{i}}(\omega)\right| \leq \sum_{j=1}^{i} \frac{2}{m_{i}} \sum_{k \geq 1} g_{i}\left(n_{k}^{i}\right)<\infty .
$$

This proves the lemma and the Gibbs representation theorem 3.39.

Remark 3.57 (Telescoping procedure). To get an idea of the type of telescoping that has to be done, we informally detail it starting from the expression (3.48) of the Hamiltonian with free boundary condition, in order to see how consistency is important to get it. It is also the way the procedure is done in an adaptation to generalized Gibbs measures in Chapter 5 to get weakly Gibbsian measures, see [99, 97, 98, 23], and we also use a similar procedure in [78] to get a variational principle for translation-invariant quasilocal measures.

Let us start from the Hamiltonian with free boundary condition for some $\Lambda$ containing the origin and assume $\Lambda$ to be a cube $\Lambda_{n_{l}}$ of the subsequence already taken, and write $L_{n_{l}}$ for the annulus $\Lambda_{n_{l}} \backslash \Lambda_{n_{l-1}}$. One has by consistency and (3.48)

$$
H_{\Lambda_{n_{l}}}^{\Phi^{+}, f}(\sigma)=\ln \frac{f_{\Lambda_{n_{l}}}(+)}{f\left(\sigma_{\Lambda_{n_{l}}}+\Lambda_{n_{l}}^{c}\right)} .
$$

The idea now is to telescope this term by incorporating terms corresponding to so-called relative-energies by flipping the spin in the annulus only

$$
H_{\Lambda_{n_{l}}}^{\Phi^{+}, f}(\sigma)=\ln \frac{f_{\Lambda_{n_{l}}}(+)}{f_{\Lambda_{n_{l}}}\left(+{ }_{\Lambda_{n_{l-1}}} \sigma_{L_{n_{l}-1}}+{ }_{\Lambda_{n_{l}}^{c}}\right)} \cdot \frac{f_{\Lambda_{n_{l}}}\left(++_{\Lambda_{n_{l-1}}} \sigma_{L_{n_{l-1}}}+{ }_{\Lambda_{n_{l}}^{c}}\right)}{f_{\Lambda_{n_{l}}}\left(++_{\Lambda_{n_{l-2}}} \sigma_{L_{n_{l-2}}}+{ }_{\Lambda_{n_{l-1}}^{c}}\right)} .
$$


Doing it for any $k=1 \ldots l$, one gets

$H_{\Lambda_{n_{l}}}^{\Phi^{+}, f}(\sigma)=\sum_{k=1}^{l} \ln \frac{f_{\Lambda_{n_{l}}}\left(+{ }_{\Lambda_{n_{k}}} \sigma_{L_{n_{k}+1}}+{\Lambda_{n_{k+1}}^{c}}\right)}{f_{\Lambda_{n_{l}}}\left(+{ }_{\Lambda_{n_{k-1}}} \sigma_{L_{n_{k}}}+{ }_{\Lambda_{n_{k}}^{c}}\right)} \cdot \frac{f_{\Lambda_{n_{l}}}\left(+{ }_{\Lambda_{n_{k-1}}} \sigma_{L_{n_{k}}}+{ }_{\Lambda_{n_{k}}^{c}}\right)}{f_{\Lambda_{n_{l}}}\left(+{ }_{\Lambda_{n_{k-2}}} \sigma_{L_{n_{k-1}}}+{ }_{\Lambda_{n_{k-1}}^{c}}\right)}$.

Now using consistency via our key lemma 3.27 , one can replace the densities $f_{\Lambda}$ by densities corresponding to the proper annulus in order to get the measurability conditions for the potential; To see this, one rewrites thus

$H_{\Lambda_{n_{l}}}^{\Phi^{+}, f}(\sigma)=\sum_{k=1}^{l} \ln \frac{f_{\Lambda_{n_{k}}}\left(+{ }_{\Lambda_{n_{k}}} \sigma_{L_{n_{k}+1}}+\Lambda_{n_{k+1}}^{c}\right) f_{\Lambda_{n_{k-1}}}\left(++_{\Lambda_{n_{k-1}}} \sigma_{L_{n_{k}}}+{ }_{\Lambda_{n_{k}}^{c}}\right)}{f_{\Lambda_{n_{k}}}\left(+{ }_{\Lambda_{n_{k-1}}} \sigma_{L_{n_{k}}}+{ }_{\Lambda_{n_{k}}^{c}}\right) f_{\Lambda_{n_{k-1}}}\left(+{ }_{\Lambda_{n_{k-2}}} \sigma_{L_{n_{k-1}}}+{ }_{\Lambda_{n_{k-1}}^{c}}\right)}$

to eventually get a potential of the form $\Psi_{L_{n_{k}}}=\Phi_{\Lambda_{n_{k}}}-\Phi_{\Lambda_{n_{k-1}}}$ for which consistency holds together with the required convergence property. We shall use such a procedure, using the expression of (3.28) in terms of $\gamma$ instead of $f$ in Chapter 4 .

Remark 3.58 (Translation-invariance and Kozlov vs. Sullivan's results). The procedure due to Kozlov to introduce its UAC potential does not yield a translation-invariant one, as explicitly seen in the sitedependent way of re-ordering terms of the Hamiltonian with free boundary condition. It is nevertheless possible to consider larger rectangles partitioning $\Lambda$ similarly for all site considered, but they have to be larger and require a condition a bit stronger than quasilocality. It is an open question whether this condition is technical or not, but Sullivan [117] has observed that the vacuum potential, in addition to be convergent and translationinvariant, is relatively uniformly convergent in the sense that the series

$$
\sum_{A \cap \Lambda \neq \emptyset, A \in \mathcal{S}}\left|\Phi_{A}^{+}(\omega)-\Phi_{A}^{+}\left(\omega_{\Lambda}^{\prime} \omega_{\Lambda^{c}}\right)\right|<+\infty
$$

are uniformly convergent in $\left(\omega, \omega^{\prime}\right)$, and also that this was enough to get quasilocality of the Gibbs specification. For the vacuum potential itself and $\omega^{\prime}=+$, this implies the usual uniform convergence of the vacuum potential. Reciprocally, any quasilocal specification has a relatively uniformly convergent potential and, although this convergence is too weak to get all the flavor of the Gibbsian theory (see again the discussion in [36]), we shall use it to derive thermodynamical properties for these measures. 


\section{Chapter 4}

\section{Equilibrium approach}

We present now an alternative, more physical, approach to describe equilibrium states at infinite volume which will eventually appear to be partially equivalent to the DLR construction presented above. Inspired by the second law of thermodynamics, described at finite volume in the introduction of Chapter 3, this so-called equilibrium approach to Gibbs measures provides thermodynamic functions at infinite volume and yields afterwards infinite-volume counterparts of the second law of thermodynamics in terms of zero relative entropy or in terms of minimization of free energy. This approach is restricted to a translation-invariant framework, mostly because it is mainly untractable otherwise ${ }^{1}$, and we shall characterize the translationinvariant equilibrium states of a given system in terms of variational principles, either specification-dependent or specification-independent depending on the choice made to characterize of the second law, as we shall see. We thus describe, in a rather general framework that will be useful for generalized Gibbs measures in the next chapter, how this approach is in some sense equivalent to the DLR approach restricted to translation-invariant measures, and describe the general proof given in [78] of the recent result that a (specification-dependent) variational principle holds for translationinvariant quasilocal specifications in general.

In all this chapter, we also restrict the infinite-volume limit procedure by mostly considering the limit $\Lambda \uparrow \mathcal{S}$ along sequences of cubes $\left(\Lambda_{n}\right)_{n \in \mathbb{N}}$, or at least sequences s.t. the ratio (surface boundary)/(volume) $\frac{|\partial \Lambda|}{|\Lambda|}$ goes to 0 , within the so-called thermodynamic limit $^{2}$. For this reason, we focus on the $d$-dimensional regular lattice $S=\mathbb{Z}^{d}$, because this thermodynamic limit does not hold for cubes on trees ${ }^{3}$, the other lattices sometimes consid-

\footnotetext{
${ }^{1}$ Except in a few situations, with e.g. periodic boundary conditions $[93,110]$.

${ }^{2}$ Although this thermodynamic is a bit more general [36, 64].

${ }^{3}$ The ratio (surface boundary)/(volume) do not vanish in the limit, see also [15].
} 
ered in these notes. We also incorporate the temperature in the potential when dealing with Gibbs specifications and measures, or equivalently here we assume $\beta=1$. At the end of the chapter, we briefly mention related large deviation properties for the considered measures and introduce another way to consider Gibbs measures as equilibrium states of the system. This dynamical construction defines them as invariant measures of Markov processes on the configuration space, and we illustrate this notion by the so-called stochastic Ising models, that will also be discussed in the generalized Gibbs framework in Chapter 5.

\subsection{Thermodynamic properties}

\subsubsection{Thermodynamic functions}

We have already introduced in Chapter 3 the relative entropy at finite volume $\Lambda \in \mathcal{S}$ of $\mu$ relative to $\nu$ for two translation-invariant measures $\mu, \nu \in \mathcal{M}_{1, \text { inv }}^{+}(\Omega)$, defined to be

$$
\mathcal{H}_{\Lambda}(\mu \mid \nu)=\int_{\Omega}\left(\frac{d \mu_{\Lambda}}{d \nu_{\Lambda}}\right) \cdot \log \left(\frac{d \mu_{\Lambda}}{d \nu_{\Lambda}}\right) d \nu
$$

when the projection $\mu_{\Lambda}$ of $\mu$ on $\left(\Omega_{\Lambda}, \mathcal{F}_{\Lambda}\right)$ is absolutely continuous w.r.t. the projection $\nu_{\Lambda}$ of $\nu$, and to be $\mathcal{H}_{\Lambda}(\mu \mid \nu)=+\infty$ otherwise. To avoid trivial divergences at infinite volume, one considers quantities per unit of volume, writes for any $n \in \mathbb{N}$

$$
h_{n}(\mu \mid \nu):=\frac{1}{\left|\Lambda_{n}\right|} \sum_{\sigma_{\Lambda_{n}} \in \Omega_{\Lambda_{n}}} \mu\left(\sigma_{\Lambda_{n}}\right) \cdot \log \frac{\mu\left(\sigma_{\Lambda_{n}}\right)}{\nu\left(\sigma_{\Lambda_{n}}\right)} .
$$

and introduces the relative entropy density of $\mu$ relative to $\nu$ to be the limit

$$
h(\mu \mid \nu)=\lim _{n \rightarrow \infty} h_{n}(\mu \mid \nu)
$$

provided it exists. The limit is known to exist for any arbitrary $\mu \in$ $\mathcal{M}_{1, \text { inv }}^{+}(\Omega)$ when $\nu \in \mathcal{M}_{1, \text { inv }}^{+}(\Omega)$ is a Gibbs measure (for a UAC potential) and, more generally, if $\nu$ is asymptotically decoupled ${ }^{4}$. We extend this result in next section for general translation-invariant quasilocal measures in Theorem 4.32, whose proof follows [78]. We also recall (see e.g. [6]) that for $\mu \in \mathcal{M}_{1, \text { inv }}^{+}(\Omega)$, the entropy per unit of volume

$$
h_{n}(\mu)=-\frac{1}{\left|\Lambda_{n}\right|} \sum_{\sigma_{\Lambda_{n}}} \mu\left(\sigma_{\Lambda_{n}}\right) \log \mu\left(\sigma_{\Lambda_{n}}\right)
$$

\footnotetext{
${ }^{4}$ These are measures introduced by Pfister [104] to state general large deviation principles, see next chapter.
} 
has a well-defined limit

$$
h(\mu):=-\lim _{n \rightarrow \infty} \frac{1}{\left|\Lambda_{n}\right|} \sum_{\sigma_{\Lambda_{n}}} \mu\left(\sigma_{\Lambda_{n}}\right) \log \mu\left(\sigma_{\Lambda_{n}}\right)
$$

called the Kolmogorov-Sinai entropy $y^{5}$ of $\mu$. For $\nu \in \mathcal{M}_{1, \text { inv }}^{+}(\Omega)$ and $f \in \mathcal{F}$ bounded, the pressure for $f$ relative to $\nu$ is defined as

$$
p(f \mid \nu)=\lim _{n \rightarrow \infty} \frac{1}{\left|\Lambda_{n}\right|^{d}} \log \int \exp \left(\sum_{x \in \Lambda_{n}} \tau_{x} f\right) d \nu
$$

whenever this limit exists. This limit exists, for every quasilocal function $f$, if $\nu$ is Gibbsian [36, 52] or asymptotically decoupled [104].

When dealing with a translation-invariant potential $\Phi$, the particular choice of

$$
f=f_{\Phi}:=\sum_{A \ni 0} \frac{1}{|A|} \cdot \Phi_{A}
$$

and with the a priori product measure $\rho$ as reference measure connects with the usual pressure in the case of a lattice gas and is more generally related with the free energy of a system obtained from a partition function with boundary condition $\omega$ or with free boundary condition. At finite volume $\Lambda_{n}$ they are respectively defined to be

$$
P_{\Lambda_{n}}^{f}(\Phi)=\frac{1}{\left|\Lambda_{n}\right|} \ln Z_{\Lambda_{n}}^{\Phi, f} \quad \text { and } \quad P_{\Lambda_{n}}^{\omega}(\Phi)=\frac{1}{\left|\Lambda_{n}\right|} \ln Z_{\Lambda_{n}}^{\Phi}(\omega) .
$$

When the limit exists, it captures many information of the particle system ${ }^{6}$ and for UAC potentials it turns out to be independent of the boundary condition:

Theorem 4.7 (Pressure of a UAC potential [64]). Let $\Phi$ be $a$ translation-invariant $U A C$ potential. Then, the limits

$$
\lim _{n \rightarrow \infty} \frac{1}{\left|\Lambda_{n}\right|} \ln Z_{\Lambda_{n}}^{\Phi, \mathrm{f}} \quad \text { and } \quad \lim _{n \rightarrow \infty} \frac{1}{\left|\Lambda_{n}\right|} \ln Z_{\Lambda_{n}}^{\Phi}(\omega)
$$

exist, coincide for all $\omega \in \Omega$ and define the pressure of the potential $\Phi$ :

$$
P(\Phi):=\lim _{n \rightarrow \infty} P_{\Lambda_{n}}^{f}(\Phi)=\lim _{n \rightarrow \infty} P_{\Lambda_{n}}^{\omega}(\Phi)
$$

exists and is thus independent of the boundary condition $\omega \in \Omega$.

\footnotetext{
${ }^{5}$ This entropy also coincides, up to the constant $\log |E|$, to the relative entropy w.r.t. the a priori counting measure.

${ }^{6}$ This is probably the most important part of mathematical statistical mechanics that we do not develop in this course, see again [36, 64, 52].
} 
We recall briefly the philosophy of the proof of Israel [64], because we extend it in this chapter to deal with the vacuum potential of a translationinvariant quasilocal measure. This potential is translation-invariant but not UAC and we shall use its relative uniform convergence, introduced in Remark 3.58, following Sullivan [117]. The proof focuses first on finiterange potentials and using the finiteness of the range, and thus the independence of spins from sites far enough, one introduces a partition of the volume $\Lambda$ into cubes and corridors, the width of the latter being at least the range of the potential, to eventually get a factorization of the partition function up to some boundary terms that are negligible in the thermodynamic limit ${ }^{7}$. This factorization leads to sub-additivity of the logarithm of the partition function, which in turns implies the existence of the pressure. This result is then extended to general UAC potentials using the density of the finite-range potential in the Banach space of translation-invariant UAC potentials [64], and thereafter the strong UAC convergence to get the independence with the boundary condition.

We also introduce the $\nu$-specific energy of a configuration $+\in \Omega$ :

$$
e_{\nu}^{+}:=-\lim _{\Lambda \uparrow \mathbb{Z}^{d}} \frac{1}{|\Lambda|} \log \nu\left(+_{\Lambda}\right)
$$

whenever it exists. We prove its existence for translation-invariant quasilocal measures in this chapter in order to get a general variational principle for translation-invariant quasilocal measures. We emphasize the fact that it is not properly speaking ${ }^{8}$ the infinite-volume counterpart of the energies of Chapter 3.

\subsubsection{Variational principles}

To translate into a proper mathematical framework the second law of thermodynamics in the vein of the finite-volume description given in Chapter 3, we distinguish between thermodynamical (specification-independent) and statistical mechanical (specification-dependent) variational principles.

Definition 4.11 (Thermodynamic variational principle). $\nu \in$ $\mathcal{M}_{1, \text { inv }}^{+}(\Omega)$ is said to satisfy a (thermodynamical) variational principle if the relative entropy $h(\mu \mid \nu)$ and the pressure $p(f \mid \nu)$ exist for all $\mu \in$ $\mathcal{M}_{1, \text { inv }}^{+}(\Omega)$ and all $f \in \mathcal{F}_{\mathrm{qloc}}$, and are conjugate convex functions in the sense that

$$
\forall f \in \mathcal{F}_{\text {qloc }}, \quad p(f \mid \nu)=\sup _{\mu \in \mathcal{M}_{1, \text { inv }}^{+}(\Omega)}[\mu(f)-h(\mu \mid \nu)] .
$$

\footnotetext{
${ }^{7}$ This is the reason why this restriction on the ratio (surface boundary)/(volume) is made in this approach.

${ }^{8}$ For a vacuum potential with vacuum state + , and a quasilocal specification, it even coincides with the pressure, see the proof of Lemma 4.33 .
} 


$$
\forall \mu \in \mathcal{M}_{1, \text { inv }}^{+}(\Omega), \quad h(\mu \mid \nu)=\sup _{f \in \mathcal{F}_{\text {qloc }}}[\mu(f)-p(f \mid \nu)] .
$$

This is the infinite-volume counterpart of the formulation of the second law of thermodynamics in terms of the minimization of free energy, which coincides with the pressure here. Gibbs measures satisfy this specificationindependent principle and Pfister [104] has extended its validity to the larger class of asymptotically decoupled measures, described in the next chapter within the generalized Gibbsian framework. These conjugate convex functions are also very important to study large deviation properties of DLR measures. We do not focus much on this type of variational principle in these lectures, and prefer focusing on the other formulation of the second law of thermodynamics, in terms of zero relative entropy.

Definition 4.14 (Variational principle relative to a specification). Consider a specification $\gamma$ and $\nu \in \mathcal{G}_{\text {inv }}(\gamma)$. A variational principle occurs for $(\nu, \gamma)$ iff

$$
\forall \mu \in \mathcal{M}_{1, \text { inv }}^{+}(\Omega), h(\mu \mid \nu)=0 \Longleftrightarrow \mu \in \mathcal{G}_{\text {inv }}(\gamma) .
$$

Hence, this property has to be related to the formulation of the second law of thermodynamics in terms of zero relative entropy, which at finite volume implies equality of measures, as explained in the beginning of Chapter 3. At infinite volume nevertheless, two different measures could have zero relative entropy, but when the reference measure has some nice locality properties ${ }^{9}$, the other measure, although possibly different, should share the same system of conditional probabilities, identifying the corresponding measures as equilibrium states of the system. This result is well known for Gibbs measures consistent with a translation-invariant UAC potential [52] and has thus been extended recently to translation-invariant quasilocal DLR measures [78]. We describe now this result under a more general form, useful for its extension to non-Gibbsian and non-quasilocal measures in Chapter 5 .

\subsection{Topological criterion for variational prin- ciples}

In this section, we consider specifications in the general framework of Chapter 2, non necessarily Gibbsian or quasilocal, and use some specific concentration properties on some points of (sometimes partial) continuity of

\footnotetext{
${ }^{9}$ Like e.g. being Gibbs. To get a counterexample, i.e. to get two measures having zero relative entropy without having much in common, consider the voter model in dimension $d=3$, with its Dirac invariant measure as a reference measure.
} 
the specification. First, we introduce the set $\Omega_{\gamma}$ of good configurations of $\gamma$ is the set of points of continuity, i.e.

$$
\begin{aligned}
\Omega_{\gamma}=\left\{\omega \in \Omega: \forall \Lambda \in \mathcal{S}, \forall f \in \mathcal{F}_{\text {loc }},\right. \\
\left.\lim _{n \rightarrow \infty} \sup _{\sigma \in \Omega}\left|\gamma_{\Lambda} f\left(\omega_{\Lambda_{n}} \sigma_{\Lambda_{n}^{c}}\right)-\gamma_{\Lambda} f(\omega)\right|=0\right\} .
\end{aligned}
$$

We also consider points of continuity in some specific direction, say $+\in \Omega$. Introduce, for all $n \in \mathbb{N}$, the truncated kernels $\gamma_{\Lambda}^{n,+}$ defined for all $f \in \mathcal{F}_{\text {loc }}$ and all $\Lambda \in \mathcal{F}$ by

$$
\forall \omega \in \Omega, \gamma_{\Lambda}^{n,+} f(\omega)=\gamma_{\Lambda} f\left(\omega_{\Lambda_{n}}+\Lambda_{n}^{c}\right)
$$

The set of points ${ }^{10}$ of continuity in the +-direction is then defined to be

$$
\Omega_{\gamma}^{+}=\left\{\omega \in \Omega: \forall \Lambda \in \mathcal{S}, \forall f \in \mathcal{F}_{\mathrm{loc}}, \lim _{n \rightarrow \infty} \gamma_{\Lambda}^{n,+} f(\omega)=\gamma_{\Lambda} f(\omega)\right\}
$$

For presumably ${ }^{11}$ technical reasons due to some telescoping procedure, we introduce also the set of configurations $\sigma$ for which there is continuity at some particular concatenated configuration $\sigma^{+} \in \Omega$ defined for all $\sigma \in \Omega$ by

$$
\sigma_{i}^{+}=\sigma_{i} \quad \text { if } \quad i \geq 0, \text { and } \sigma_{i}=+_{i} \text { otherwise. }
$$

We denote this set by

$$
\Omega_{\gamma}^{<0}=\left\{\sigma \in \Omega: \sigma^{+} \in \Omega_{\gamma}\right\} .
$$

Due to the definition of these sets by a limiting procedure, involved in any continuity-type property, one can prove that these sets are tail-measurable and such that

$$
\Omega_{\gamma} \subset \Omega_{\gamma}^{+} \subset \Omega_{\gamma}^{<0} \in \mathcal{F}_{\infty}
$$

\subsubsection{Second part of the variational principle: General criterion}

Getting consistency from zero relative entropy is seen as the "easiest part", usually called the second part. The result and its proof are standard, but we give a slightly more general version of both, that will be useful also for non-quasilocal measures in Chapter 5 . What is actually really needed is some weaker continuity in the + -direction for some reference configuration $+\in \Omega$, as we see now.

\footnotetext{
${ }^{10}$ Remark that a function can be continuous in any direction + without being continuous, see [46].

${ }^{11}$ See the telescoping procedure in next section and a discussion in Chapter 5.
} 
Theorem 4.21. [45, 46] Let $\gamma$ be a specification that is quasilocal in the direction $+\in \Omega$, and $\nu \in \mathcal{G}_{\text {inv }}(\gamma)$. If $\mu \in \mathcal{M}_{1}^{+}(\Omega)$ is such that $h(\mu \mid \nu)=0$, then

$$
\mu \in \mathcal{G}(\gamma) \Longleftrightarrow \nu\left[g_{\Lambda_{n} \backslash \Lambda} \cdot\left(\gamma_{\Lambda}^{n,+} f-\gamma_{\Lambda} f\right)\right] \underset{n \rightarrow \infty}{\longrightarrow} 0
$$

for all $\Lambda \in \mathcal{S}$ and $f \in \mathcal{F}_{\text {loc }}$, where $g_{\Lambda_{n} \backslash \Lambda}:=\frac{d \mu_{\Lambda_{n} \backslash \Lambda}}{d \nu_{\Lambda_{n} \backslash \Lambda}}$ provided it exists.

Thus, to get information about consistency from zero relative entropy requires that the concentration properties of the density of $\mu_{\Lambda_{n} \backslash \Lambda}$ w.r.t $\nu_{\Lambda_{n} \backslash \Lambda}$ to beat asymptotic divergence due to the lack of continuity of $\gamma$. When $\gamma$ is quasilocal, this lack of continuity never exists $\left(\Omega_{\gamma}=\Omega\right)$ and is thus always beaten, yielding the standard proof of the second part of the variational principle for translation-invariant quasilocal specifications.

Proof of Theorem 4.21: It comes from [45, 46] and is an adaptation of the standard proof $[52,104]$ in the quasilocal case, where the criterion (4.22) is trivially valid. When $h(\mu \mid \nu)=0$ holds, the latter relative entropy is in particular well-defined and as a consequence, for $n$ sufficiently large, the $\mathcal{F}_{\Lambda_{n}}$-measurable density $g_{\Lambda_{n}}:=d \mu_{\Lambda_{n}} / d \nu_{\Lambda_{n}}$ exists. Fix $f \in \mathcal{F}_{\text {loc }}, \Lambda \in \mathcal{S}$ and pick $n$ big enough to get both $\Lambda_{n} \supset \Lambda$ and the existence of $g_{\Lambda_{n}}$. To prove that $\mu \in \mathcal{G}(\gamma)$, we prove that $\mu \gamma_{\Lambda}[f]=\mu[f]$, and approximate first $\gamma$ by the truncated kernel (4.17), writing

$$
\mu \gamma_{\Lambda}[f]=\mu \gamma_{\Lambda}^{n,+}[f]+A_{n}
$$

where $A_{n}=\mu\left[\gamma_{\Lambda} f-\gamma_{\Lambda}^{n,+} f\right]$ goes to zero when $n$ goes to infinity by continuity in the +-direction. Now we can use consistency, the $\mathcal{F}_{\Lambda_{n} \backslash \Lambda^{-}}$ measurability of the truncated kernel $\gamma_{\Lambda}^{n,+}[f]$ and the $\mathcal{F}_{\Delta}$-measurability of any density $g_{\Delta}$ to rewrite

$$
\mu \gamma_{\Lambda}^{n,+}[f]=\mu\left[\gamma_{\Lambda}^{n,+} f\right]=\nu\left[g_{\Lambda_{n} \backslash \Lambda} \cdot \gamma_{\Lambda}^{n,+} f\right]=\nu\left[g_{\Lambda_{n} \backslash \Lambda} \cdot \gamma_{\Lambda} f\right]+B_{n},
$$

where

$$
B_{n}=\nu\left[g_{\Lambda_{n} \backslash \Lambda} \cdot\left(\gamma_{\Lambda}^{n,+} f-\gamma_{\Lambda} f\right)\right]
$$

is controlled by the criterion (4.22). Now, by $\mathcal{F}_{\Lambda^{c}}$-measurability of $g_{\Lambda_{n} \backslash \Lambda}$ and consistency of $\nu$, one rewrites

$$
\nu\left[g_{\Lambda_{n} \backslash \Lambda} \cdot \gamma_{\Lambda} f\right]=\nu\left[\gamma_{\Lambda}\left(g_{\Lambda_{n} \backslash \Lambda} \cdot f\right)\right]=\nu\left[g_{\Lambda_{n} \backslash \Lambda} \cdot f\right],
$$

which has to be compared with

$$
\mu[f]=\nu\left[g_{\Lambda_{n}} \cdot f\right]=\nu\left[g_{\Lambda_{n} \backslash \Lambda} \cdot f\right]+C_{n}
$$

with

$$
C_{n}=\nu\left[\left(g_{\Lambda_{n} \backslash \Lambda}-g_{\Lambda_{n}}\right) \cdot f\right] .
$$


We can evaluate $C_{n}$ using the following inequality due to Csiszár [20]

$\left|C_{n}\right|=\left|\nu\left[\left(g_{\Lambda_{n}}-g_{\Lambda_{n} \backslash \Lambda}\right) f\right]\right| \leq\left(2 \sup _{\omega \in \Omega}|f(\omega)|\left(H_{\Lambda_{n}}(\mu \mid \nu)-H_{\Lambda_{n} \backslash \Lambda}(\mu \mid \nu)\right)\right)^{1 / 2}$.

From the hypothesis of zero relative entropy, which implies that the finitevolume relative entropy cannot grow faster than the volume, one also has a subvolumic rate of deacreasing for the difference of densities of the r.h.s. above, and eventually gets that $\mu \gamma_{\Lambda}[f]=\mu[f]$ if and only if $B_{n} \underset{n \rightarrow \infty}{\longrightarrow} 0$, which proves the lemma.

This criterion is a way to express that getting zero relative entropy is meaningful only when the measures share some locality properties. Without properties of that type, things could be different, as illustrated by an example of [120]. This criterion has been upgraded in [41] via the following theorem, also useful for the generalized Gibbs measures.

Theorem 4.23. [41] Let $\mu \in \mathcal{G}(\gamma)$ and $\nu \in \mathcal{M}_{1}^{+}(\Omega)$ such that $h(\mu \mid \nu)=0$. If, for all $\sigma \in \Omega$, for $\nu$-a.e. $\omega$,

$$
\mu\left(\sigma_{\Lambda} \mid \omega_{\Lambda_{n} \backslash \Lambda}\right) \underset{n \rightarrow \infty}{\longrightarrow} \gamma_{\Lambda}(\sigma \mid \omega)
$$

then $\nu \in \mathcal{G}(\gamma)$.

\subsubsection{First part of the variational principle: General criterion}

In the usual theory of Gibbs measures, this part of the variational principle, i.e getting zero relative entropy from consistency, is known when the latter holds with a translation-invariant specification defined via a translation-invariant $U A C$ potential, and goes via existence and boundary condition independence of pressure (see [52]). Since for a general translation-invariant quasilocal specification $\gamma$ we cannot rely on the existence of such a translation-invariant potential, we shall use the weaker property of relative uniform convergence of the (translation-invariant) vacuum potential which can be associated to the quasilocal, see Remark 3.58, is enough to obtain zero relative entropy. We get this result as a consequence of more general results on generalized Gibbs measures developed in [78] that will be useful in the next chapter.

We consider a translation-invariant specification $\gamma$ and a probability measure $\nu \in \mathcal{G}_{\text {inv }}(\gamma)$.

Theorem 4.24. [78] If $\mu \in \mathcal{M}_{1, \text { inv }}^{+}(\Omega)$ is such that $\mu\left(\Omega_{\gamma}^{<0}\right)=1$ and $e_{\nu}^{+}$ exists, then 
1. $h(\mu \mid \nu)$ exists and is given by

$$
h(\mu \mid \nu)=-h(\mu)+e_{\nu}^{+}-\int_{\Omega} \log \frac{\gamma_{0}\left(\sigma^{+} \mid \sigma^{+}\right)}{\gamma_{0}\left(+\mid \sigma^{+}\right)} \mu(d \sigma) .
$$

2. If moreover $\mu \in \mathcal{G}_{\text {inv }}(\gamma)$ exists, then

$$
h(\mu \mid \nu)=\lim _{\Lambda \uparrow \mathbb{Z}^{d}} \frac{1}{|\Lambda|} \log \frac{\mu\left(+_{\Lambda}\right)}{\nu\left(+_{\Lambda}\right)} .
$$

To prove this theorem, we establish a more general lemma that will help to restore the thermodynamic properties of generalized Gibbs in Chapter 5. It topologically captures what is needed for a specification to get zero relative entropy of its DLR measures. The full proof is given in [78].

Lemma 4.27. [78] If $\mu\left(\Omega_{\gamma}^{<0}\right)=1$, then

1. Uniformly in $\omega \in \Omega$,

$$
\lim _{n \rightarrow \infty} \frac{1}{\left|\Lambda_{n}\right|} \int_{\Omega} \log \frac{\gamma_{\Lambda_{n}}(\sigma \mid \omega)}{\gamma_{\Lambda_{n}}(+\mid \omega)} \mu(d \sigma)=\int_{\Omega} \log \frac{\gamma_{0}\left(\sigma^{+} \mid \sigma^{+}\right)}{\gamma_{0}\left(+\mid \sigma^{+}\right)} \mu(d \sigma) .
$$

2. For $\nu \in \mathcal{G}(\gamma)$,

$$
\lim _{n \rightarrow \infty} \frac{1}{\left|\Lambda_{n}\right|} \int_{\Omega} \log \frac{\nu\left(\sigma_{\Lambda_{n}}\right)}{\nu\left({ }_{\Lambda_{n}}\right)} \mu(d \sigma)=\int_{\Omega} \log \frac{\gamma_{0}\left(\sigma^{+} \mid \sigma^{+}\right)}{\gamma_{0}\left(+\mid \sigma^{+}\right)} \mu(d \sigma) .
$$

In particular, the limit depends only on the pair $(\gamma, \mu)$.

\section{Proof :}

1. The proof relies on the uniform convergence of the translationinvariant vacuum potential with vacuum state + established by Sullivan using a particular telescoping procedure. This procedure is described in Remark 3.57 under the assumption of quasilocality but it is not difficult to extend its validity when only almost-sure continuity in the + direction holds, which is in particular implied by the condition $\mu\left(\Omega_{\gamma}^{<0}\right)=1$. This (not optimal ${ }^{12}$ ) latter condition comes from the telescoping procedure used here, and we do not know whether it is technical or not. In particular, we do not know if it can be relaxed to a general almost-sure quasilocality property, a very important property in the context of generalized Gibbs measures. Following Sullivan [116], we define, for all $\sigma \in \Omega$

$$
D(\sigma)=E_{\{0\}}^{+}(\sigma \mid \sigma)=\log \frac{\gamma_{0}(\sigma \mid \sigma)}{\gamma_{0}(+\mid \sigma)} .
$$

\footnotetext{
${ }^{12}$ It is indeed extended in [78].
} 
and consider an approximation of $\sigma^{+}$at finite volume $\Lambda \in \mathcal{S}$ with boundary condition $\omega$ by defining the telescoping configuration at site $i \in S$ to be $T_{\Lambda}^{\omega}[i, \sigma,+]$, defined for all $j \in S$ by:

$$
\left(T_{\Lambda}^{\omega}[x, \sigma,+]\right)_{j}=\left\{\begin{array}{lll}
\omega_{j} & \text { if } & j \in \Lambda^{c} \\
\sigma_{j} & \text { if } j \leq i, j \in \Lambda \\
+1 & \text { if } j>i, j \in \Lambda
\end{array}\right.
$$

To perform the telescoping, denote $\Lambda_{\leq i}=\{j \in \Lambda: j \leq i\}, \Lambda_{<i}=\Lambda_{\leq i} \backslash\{i\}$, $\Lambda_{>i}=\Lambda \backslash \Lambda_{\leq i}$ and let $\Lambda=\left\{i_{1}, \ldots i_{N}\right\}$ denote an enumeration in the lexicographic order. By consistency and Lemma 3.27:

$$
\frac{\gamma_{\Lambda}(\sigma \mid \omega)}{\gamma_{\Lambda}(+\mid \omega)}=\prod_{k=1}^{N} \frac{\gamma_{\Lambda}\left(\sigma_{\Lambda_{\leq i_{k}}}+{ }_{\Lambda_{>i_{k}}} \mid \omega\right)}{\gamma_{\Lambda}\left(\sigma_{\Lambda_{\leq i_{k-1}}}+{ }_{\Lambda_{>i_{k-1}}} \mid \omega\right)}=\prod_{k=1}^{N} \frac{\gamma_{i_{k}}\left(\sigma_{i_{k}} \mid \sigma_{\Lambda_{<i_{k}}}+{ }_{\Lambda_{>i_{k}}} \omega_{\Lambda^{c}}\right)}{\gamma_{i_{k}}\left(+_{i_{k}} \mid \sigma_{\Lambda_{<i_{k}}}+{ }_{\Lambda_{>i_{k}}} \omega_{\Lambda^{c}}\right)}
$$

Taking the logarithm yields for $\Lambda=\Lambda_{n}$

$$
\int_{\Omega} \log \frac{\gamma_{\Lambda_{n}}(\sigma \mid \omega)}{\gamma_{\Lambda_{n}}(+\mid \omega)} \mu(d \sigma)=\sum_{i \in \Lambda_{n}} \int_{\Omega} D\left(\tau_{-i} T_{\Lambda_{n}}^{\omega}\left[i, \tau_{i} \sigma,+\right]\right) \mu(d \sigma)
$$

in such a way that proving Item 1 . amounts to prove that, uniformly in $\omega$, the r.h.s divided by the volume converges to $\int_{\Omega} D\left(\sigma^{+}\right) \mu(d \sigma)$. To do so in [78], we carefully count the points of the set $A_{n}$ where this telescoping configuration $T_{\Lambda}^{\omega}[i, \sigma,+]$ and $\sigma^{+}$differ, to establish that $\left|A_{n}\right|=\circ\left(\left|\Lambda_{n}\right|\right)$ and to eventually get, due to the continuity of $D$ at the configuration $\sigma^{+}$, that for all $\epsilon>0$

$$
\frac{1}{\left|\Lambda_{n}\right|}\left|\sum_{i \in \Lambda_{n}}\left[D\left(\tau_{-i} T_{\Lambda_{n}}^{\omega}\left[i, \tau_{x} \sigma,+\right]\right)-D\left(\sigma^{+}\right)\right]\right| \leq \epsilon+2 \sup _{\omega}|D(\omega)| \cdot \frac{\left|A_{n}\right|}{\left|\Lambda_{n}\right|}
$$

which is less than $2 \epsilon$ for $n$ big enough. So we obtain that

$$
\frac{1}{\left|\Lambda_{n}\right|}\left|\sum_{i \in \Lambda_{n}}\left[D\left(\tau_{-i} T_{\Lambda_{n}}^{\omega}\left[i, \tau_{x} \sigma,+\right]\right)-D\left(\sigma^{+}\right)\right]\right|
$$

converges to zero on the set of $\Omega_{\gamma}^{<0}$ of full $\mu$-measure, uniformly in $\omega$, which implies statement 1 of the lemma by dominated convergence.

2. Since $\nu \in \mathcal{G}(\gamma)$, by consistency one rewrites for all $\sigma \in \Omega$

$$
\nu\left(\sigma_{\Lambda_{n}}\right)=\int_{\Omega} \gamma_{\Lambda_{n}}(\sigma \mid \omega) \nu(d \omega)
$$

so that if one denotes $F_{\Lambda_{n}}(\mu, \nu):=\frac{1}{\left|\Lambda_{n}\right|} \int_{\Omega} \log \frac{\nu\left(\sigma_{\Lambda_{n}}\right)}{\nu\left(+_{\Lambda_{n}}\right)} \mu(d \sigma)$, one can rewrite

$$
F_{\Lambda_{n}}(\mu, \nu)=\frac{1}{\left|\Lambda_{n}\right|} \int_{\Omega} \log \frac{\int_{\Omega} \gamma_{\Lambda_{n}}(\sigma \mid \omega) \nu(d \omega)}{\int_{\Omega} \gamma_{\Lambda_{n}}(+\mid \omega) \nu(d \omega)} \mu(d \sigma)
$$


We use now the obvious bound

$$
\inf _{\omega \in \Omega} \frac{\gamma_{\Lambda_{n}}(\sigma \mid \omega)}{\gamma_{\Lambda_{n}}(+\mid \omega)} \leq \frac{\int_{\Omega} \gamma_{\Lambda_{n}}(\sigma \mid \omega) \nu(d \omega)}{\int_{\Omega} \gamma_{\Lambda_{n}}(+\mid \omega) \nu(d \omega)} \leq \sup _{\omega \in \Omega} \frac{\gamma_{\Lambda_{n}}(\sigma \mid \omega)}{\gamma_{\Lambda_{n}}(+\mid \omega)}
$$

to get that for any $\epsilon>0$ there exists $\omega=\omega(n, \sigma, \epsilon), \omega^{\prime}=\omega^{\prime}(n, \sigma, \epsilon)$ such that

$$
\int_{\Omega} \inf _{\omega \in \Omega} \log \frac{\gamma_{\Lambda_{n}}(\sigma \mid \omega)}{\gamma_{\Lambda_{n}}(+\mid \omega)} \mu(d \sigma) \geq \int_{\Omega} \log \frac{\gamma_{\Lambda_{n}}(\sigma \mid \omega(n, \sigma, \epsilon))}{\gamma_{\Lambda_{n}}(+\mid \omega(n, \sigma, \epsilon))}-\epsilon
$$

and

$$
\int_{\Omega} \sup _{\omega \in \Omega} \log \frac{\gamma_{\Lambda_{n}}(\sigma \mid \omega)}{\gamma_{\Lambda_{n}}(+\mid \omega)} \mu(d \sigma) \leq \int_{\Omega} \log \frac{\gamma_{\Lambda_{n}}\left(\sigma \mid \omega^{\prime}(n, \sigma, \epsilon)\right)}{\gamma_{\Lambda_{n}}\left(+\mid \omega^{\prime}(n, \sigma, \epsilon)\right)}+\epsilon .
$$

Now use the first item of the lemma and choose $N$ such that for all $n \geq N$,

$$
\sup _{\omega}\left|\frac{1}{\left|\Lambda_{n}\right|} \int_{\Omega} \log \frac{\gamma_{\Lambda_{n}}(\sigma \mid \omega)}{\gamma_{\Lambda_{n}}(+\mid \omega)} \mu(d \sigma)-\int_{\Omega} D\left(\sigma^{+}\right) \mu(d \sigma)\right| \leq \epsilon
$$

to get, for $n \geq N$,

$$
\int_{\Omega} D\left(\sigma^{+}\right) \mu(d \sigma)-2 \epsilon \leq F_{\Lambda_{n}}(\mu \mid \nu) \leq \int_{\Omega} D\left(\sigma^{+}\right) \mu(d \sigma)+2 \epsilon
$$

and eventually prove the lemma.

\section{Proof of Theorem 4.24:}

1. By a short computation at finite volume, rewrite

$$
\begin{aligned}
h_{n}(\mu \mid \nu)=-h_{n}(\mu) & -\frac{1}{\left|\Lambda_{n}\right|} \sum_{\sigma_{\Lambda_{n}} \in \Omega_{\Lambda_{n}}} \mu\left(\sigma_{\Lambda_{n}}\right) \log \frac{\nu\left(\sigma_{\Lambda_{n}}\right)}{\nu\left(+_{\Lambda_{n}}\right)} \\
& -\frac{1}{\left|\Lambda_{n}\right|} \log \nu\left(+{ }_{\Lambda_{n}}\right) .
\end{aligned}
$$

When $\mu\left(\Omega_{\gamma}^{<0}\right)=1$ holds, the asymptotic behavior of the second term of the r.h.s. is given by Lemma 4.27 and under the existence of $e_{\nu}^{+}$ one gets (4.25).

2. For $\mu \in \mathcal{G}_{\text {inv }}(\gamma)$ such that $\mu\left(\Omega_{\gamma}^{<0}\right)=1$, rewrite now :

$$
\begin{aligned}
h_{n}(\mu \mid \nu) & =\frac{1}{\left|\Lambda_{n}\right|} \sum_{\sigma_{\Lambda_{n}} \in \Omega_{\Lambda_{n}}} \mu\left(\sigma_{\Lambda_{n}}\right) \log \frac{\mu\left(\sigma_{\Lambda_{n}}\right)}{\mu\left(+{ }_{\Lambda_{n}}\right)} \\
& -\frac{1}{\left|\Lambda_{n}\right|} \sum_{\sigma_{\Lambda_{n}} \in \Omega_{\Lambda_{n}}} \mu\left(\sigma_{\Lambda_{n}}\right) \log \frac{\nu\left(\sigma_{\Lambda_{n}}\right)}{\nu\left(+{ }_{\Lambda_{n}}\right)}+\frac{1}{\left|\Lambda_{n}\right|} \log \frac{\mu\left(+\Lambda_{n}\right)}{\nu\left(+{ }_{\Lambda_{n}}\right)} .
\end{aligned}
$$


By Lemma 4.27, in the limit $n \rightarrow \infty$, the first two terms of the r.h.s. are functions of $(\gamma, \mu)$ rather than functions of $\nu, \mu \in \mathcal{G}_{\text {inv }}(\gamma)$ and cancel out. Hence, the relative entropy exists if and only if the last term converges. Using Item 1 (existence of relative entropy), we obtain the existence of the limit in (4.26) and the equality

$$
h(\mu \mid \nu)=\lim _{n \rightarrow \infty} \frac{1}{\left|\Lambda_{n}\right|} \log \frac{\mu\left(+_{\Lambda_{n}}\right)}{\nu\left(+_{\Lambda_{n}}\right)}
$$

and in particular the r.h.s is a well-defined limit.

\subsubsection{Application: Variational principle for translation- invariant quasilocal measures}

Theorem 4.30. [78] Any $\mu \in \mathcal{M}_{1, \text { inv }}^{+}(\Omega)$ quasilocal satisfies the variational principle.

Proof: When $\gamma$ is quasilocal, one has $\Omega_{\gamma}=\Omega$ and the second part is a direct consequence of Theorem 4.1, because the convergence required is true by any convergence theorem (uniform or dominated). We recover thus the usual standard proof of

Theorem 4.31 (2nd part of the VP for quasilocal measures). Let $\gamma$ be a quasilocal and translation-invariant specification and $\nu \in \mathcal{G}_{\mathrm{inv}}(\gamma)$. Then for any $\mu \in \mathcal{M}_{1, \text { inv }}^{+}(\Omega)$,

$$
h(\mu \mid \nu)=0 \Longrightarrow \mu \in \mathcal{G}_{\text {inv }}(\gamma)
$$

Thus, the result of [78], that extends the variational principle from translation-invariant Gibbs measures with a translation-invariant UAC potential to translation-invariant quasilocal measures, for which the potential derived from Kozlov (and described in the previous chapter) is not necessarily UAC, relies on the following

Theorem 4.32 (1st part of the VP for quasilocal measures). Let $\gamma$ be a quasilocal and translation-invariant specification, $\nu \in \mathcal{G}_{\mathrm{inv}}(\gamma)$ and $\mu \in \mathcal{M}_{1, \text { inv }}^{+}(\Omega)$. Then $h(\mu \mid \nu)$ exists for all $\mu \in \mathcal{M}_{1, \text { inv }}^{+}(\Omega)$ and

$$
\mu \in \mathcal{G}_{\text {inv }}(\gamma) \Longrightarrow h(\mu \mid \nu)=0
$$

Proof: We need the following lemma to use Theorem 4.24:

Lemma 4.33. For $\mu, \nu \in \mathcal{G}_{\text {inv }}(\gamma)$ with $\gamma$ t.i. and quasilocal, $e_{\nu}^{+}, e_{\mu}^{+}$exist and

$$
\lim _{n \rightarrow \infty} \frac{1}{\left|\Lambda_{n}\right|} \log \frac{\mu\left(+{ }_{\Lambda_{n}}\right)}{\nu\left(+{ }_{\Lambda_{n}}\right)}=0 .
$$


Proof: For any $\mu \in \mathcal{G}(\gamma)$, one writes, using the vacuum property

$$
\begin{aligned}
\frac{1}{\left|\Lambda_{n}\right|} \log \mu\left(+_{\Lambda_{n}}\right) & =\frac{1}{\left|\Lambda_{n}\right|} \log \int_{\Omega} \frac{e^{-H_{\Lambda_{n}}^{\Phi^{+}}(+\mid \eta)}}{Z_{\Lambda_{n}}^{\Phi^{+}}(\eta)} \mu(d \eta) \\
& =\frac{1}{\left|\Lambda_{n}\right|} \log \int_{\Omega} \frac{1}{Z_{\Lambda_{n}}^{\Phi^{+}}(\eta)} \mu(d \eta) \\
& \leq \frac{1}{\left|\Lambda_{n}\right|} \log \sup _{\eta} \frac{1}{Z_{\Lambda_{n}}^{\Phi^{+}}(\eta)}
\end{aligned}
$$

and similarly

$$
\frac{1}{\left|\Lambda_{n}\right|} \log \mu\left(+\Lambda_{n}\right) \geq \frac{1}{\left|\Lambda_{n}\right|} \log \inf _{\eta} \frac{1}{Z_{\Lambda_{n}}^{\Phi+}(\eta)} .
$$

Equation (4.34) of the previous lemma is then a direct consequence of the following lemma, that relies on specific properties of the vacuum potential.

Lemma 4.35. The vacuum potential $\Phi^{+}$with vacuum state + associated with the quasilocal specification $\gamma$ is such that:

1.

$$
\lim _{n \rightarrow \infty} \sup _{\omega, \eta, \sigma} \frac{1}{\left|\Lambda_{n}\right|}\left|H_{\Lambda_{n}}^{\Phi^{+}}(\sigma \mid \eta)-H_{\Lambda_{n}}^{\Phi^{+}}(\sigma \mid \omega)\right|=0
$$

2.

$$
\lim _{n \rightarrow \infty} \sup _{\omega, \eta} \frac{1}{\left|\Lambda_{n}\right|} \log \frac{Z_{\Lambda_{n}^{+}}^{\Phi^{+}}(\omega)}{Z_{\Lambda_{n}}^{\Phi^{+}}(\eta)}=0
$$

Proof: Clearly, (4.36) implies (4.37): For all $n \in \mathbb{N}$,

$$
\begin{array}{r}
\exp \left\{-\sup _{\omega, \eta, \sigma}\left|H_{\Lambda_{n}}^{\Phi^{+}}(\sigma \mid \eta)-H_{\Lambda_{n}}^{\Phi^{+}}(\sigma \mid \omega)\right|\right\} \leq \sup _{\omega, \eta} \frac{Z_{\Lambda_{n}}^{\Phi^{+}}(\omega)}{Z_{\Lambda_{n}}^{\Phi^{+}}(\eta)} \\
\leq \exp \left\{\sup _{\omega, \eta, \sigma}\left|H_{\Lambda_{n}}^{\Phi^{+}}(\sigma \mid \eta)-H_{\Lambda_{n}}^{\Phi^{+}}(\sigma \mid \omega)\right|\right\} .
\end{array}
$$

Now one proves (4.36) by rewriting first

$$
H_{\Lambda_{n}}^{\Phi^{+}}(\sigma \mid \eta)-H_{\Lambda_{n}}^{\Phi^{+}}(\sigma \mid \omega)=\sum_{A \cap \Lambda_{n} \neq \emptyset, A \cap \Lambda_{n}^{c} \neq \emptyset}\left[\Phi_{A}^{+}\left(\sigma_{\Lambda_{n}} \eta_{\Lambda_{n}^{c}}\right)-\Phi_{A}^{+}\left(\sigma_{\Lambda_{n}} \omega_{\Lambda_{n}^{c}}\right)\right] .
$$

Unfortunately, the third inequality on page 1707 in [78] and likewise the second inequality of page 1709 are wrong in general, due to the possible non-absolute summability of the potential. Instead of them, we introduce at any volume $\Lambda_{n}$ an order $\geq$ that enumerates all sites in $\Lambda_{n}$ and then 
exhausts the rest of the lattice, and use the following formal identity, valid for any site $x$, any $n \in \mathbb{N}$, any potential $\Phi$ :

$$
\sum_{A \cap \Lambda_{n} \neq \emptyset, A \cap \Lambda_{n}^{c} \neq \emptyset} \Phi_{A}=\sum_{x \in \Lambda_{n}} \sum_{A \geq x, A \cap \Lambda_{n}^{c} \neq \emptyset} \Phi_{A} .
$$

where the notation " $A \geq x$ " denotes the sets $A$ whose first site in this order is $x$. This condition contains the former requirement $A \cap \Lambda_{n} \neq \emptyset$ and we use it to rewrite (4.38) as

$$
H_{\Lambda_{n}}^{\Phi^{+}}(\sigma \mid \eta)-H_{\Lambda_{n}}^{\Phi^{+}}(\sigma \mid \omega)=\sum_{x \in \Lambda_{n}} \sum_{A \geq x, A \cap \Lambda_{n}^{c} \neq \emptyset}\left[\Phi_{A}^{+}\left(\sigma_{\Lambda_{n}} \eta_{\Lambda_{n}^{c}}\right)-\Phi_{A}^{+}\left(\sigma_{\Lambda_{n}} \omega_{\Lambda_{n}^{c}}\right)\right]
$$

Using specific properties of the vacuum potential, namely that the configuration " + " is a vacuum state for which $\Phi_{A}^{+}(\omega)=0$ whenever $\omega_{i}=+_{i}$ for some $i \in A$, one can interpret the last sum as the difference between two vacuum Hamiltonians at two ( $x$-dependent) particular configurations, i.e. one manages to remove the condition " $A \geq x$ " in the label of the sum and recover a more standard label $A \ni x$, to eventually get

$$
\begin{aligned}
& H_{\Lambda_{n}}^{\Phi^{+}}(\sigma \mid \eta)-H_{\Lambda_{n}}^{\Phi^{+}}(\sigma \mid \omega)= \\
& \sum_{x \in \Lambda_{n}} \sum_{A \ni x, A \cap \Lambda_{n}^{c} \neq \emptyset}\left[\Phi_{A}^{+}\left(+_{<x} \sigma_{\Lambda_{n}, \geq x} \eta_{\Lambda_{n}^{c}, \geq x}\right)-\Phi_{A}^{+}\left(+_{<x} \sigma_{\Lambda_{n}, \geq x} \omega_{\Lambda_{n}^{c}, \geq x}\right)\right],
\end{aligned}
$$

where, with obvious notations, the configuration $+{ }_{<x} \sigma_{\Lambda_{n}, \geq x} \eta_{\Lambda_{n}^{c}, \geq x}$ is the configuration that coincides with the vacuum state +1 at any site strictly lower than $x$ in the chosen order and with the concatenated configuration $\sigma_{\Lambda_{n}} \eta_{\Lambda_{n}^{c}}$ otherwise. Now, to prove (4.36) using the translation-invariance and the uniform convergence of the vacuum potential, we rewrite (4.39) as

$$
H_{\Lambda_{n}}^{\Phi^{+}}(\sigma \mid \eta)-H_{\Lambda_{n}}^{\Phi^{+}}(\sigma \mid \omega)=\sum_{x \in \Lambda_{n}} \psi_{n}^{\eta, \omega}(\sigma, 0)+\sum_{x \in \Lambda_{n}}\left(\psi_{n}^{\eta, \omega}(\sigma, x)-\psi_{n}^{\eta, \omega}(\sigma, 0)\right)
$$

where for all $n \in \mathbb{N}$, for all $x \in \Lambda_{n}$ and $\omega, \eta \in \Omega$

$$
\begin{aligned}
\psi_{n}^{\eta, \omega}(\sigma, x)=\sum_{A \ni x, A \cap \Lambda_{n}^{c} \neq \emptyset}\left[\Phi_{A}^{+}(+<x\right. & \left.\sigma_{\Lambda_{n}, \geq x} \eta_{\Lambda_{n}^{c}, \geq x}\right) \\
& \left.-\Phi_{A}^{+}\left(+_{<x} \sigma_{\Lambda_{n}, \geq x} \omega_{\Lambda_{n}^{c}, \geq x}\right)\right] .
\end{aligned}
$$

If we focus on the case $x=0$ first, we recognize a difference of "outer" Hamiltonians, which goes uniformly to 0 by uniform convergence of the vacuum potential [117] (and the Cauchy property of this convergence), to eventually get uniformly in $\sigma$ 


$$
\lim _{n \rightarrow \infty} \sup _{\eta, \omega}\left|\psi_{n}^{\eta, \omega}(\sigma, 0)\right|=0 .
$$

It remains thus to use translation-invariance to estimate the difference between $\psi_{n}^{\eta, \omega}(\sigma, 0)$ and $\psi_{n}^{\eta, \omega}(\sigma, x)$, to establish that

$$
\lim _{n \rightarrow \infty} \frac{1}{\left|\Lambda_{n}\right|} \sup _{\sigma, \eta, \omega}\left|\sum_{x \in \Lambda_{n}}\left(\psi_{n}^{\eta, \omega}(\sigma, x)-\psi_{n}^{\eta, \omega}(\sigma, 0)\right)\right|=0 .
$$

Indeed, by translation-invariance one rewrites

$$
\begin{aligned}
& \psi_{n}^{\eta, \omega}(\sigma, x) \\
& =\sum_{A \ni x, A \cap \Lambda_{n}^{c} \neq \emptyset}\left[\Phi_{\tau_{-x} A}^{+}\left(+<0 \sigma_{\Lambda_{n}, \geq 0} \eta_{\Lambda_{n}^{c}, \geq 0}\right)-\Phi_{\tau_{-x} A}^{+}\left(+<0 \sigma_{\Lambda_{n}, \geq 0} \omega_{\Lambda_{n}^{c}, \geq 0}\right)\right] \\
& =\sum_{A \ni 0, A+x \cap \Lambda_{n}^{c} \neq \emptyset}\left[\Phi_{A}^{+}\left(+<0 \sigma_{\Lambda_{n}, \geq 0} \eta_{\Lambda_{n}^{c}, \geq 0}\right)-\Phi_{A}^{+}\left(+<0 \sigma_{\Lambda_{n}, \geq 0} \omega_{\Lambda_{n}^{c}, \geq 0}\right)\right]
\end{aligned}
$$

and we are reduced to control the asymptotic behavior of

$$
\frac{1}{\left|\Lambda_{n}\right|} \sum_{x \in \Lambda_{n}} \tilde{\psi}_{n}^{\eta, \omega}(\sigma, x)
$$

where $\quad \tilde{\psi}_{n}^{\eta, \omega}(\sigma, x)=$

$$
\sum_{A \ni 0, A \subset \Lambda_{n}, A+x \cap \Lambda_{n}^{c} \neq \emptyset}\left[\Phi_{A}^{+}\left(+<0 \sigma_{\Lambda_{n}, \geq 0} \eta_{\Lambda_{n}^{c}, \geq 0}\right)-\Phi_{A}^{+}\left(+<0 \sigma_{\Lambda_{n}, \geq 0} \omega_{\Lambda_{n}^{c}, \geq 0}\right)\right] .
$$

We identify it as a Cesáro mean of a sequence that is uniformly bounded and goes to zero in the infinite-volume limit:

$$
\begin{aligned}
\left|\tilde{\psi}_{n}^{\eta, \omega}(\sigma, x)\right| & \leq \sup _{\eta}\left|\Phi_{A}^{+}\left(+<0 \sigma_{\Lambda_{n}, \geq 0} \eta_{\Lambda_{n}^{c}, \geq 0}\right)\right| \\
& \leq \phi\left(d\left(x, \Lambda_{n}^{c}\right)\right),
\end{aligned}
$$

where $\phi$ is a function of the distance of $x$ and $\Lambda_{n}^{c}$ only, that goes to zero uniformly in the configurations when $n$ goes to infinity. Thus, using Cesáro's theorem, (4.44) goes to zero uniformly in the infinite-volume limit, yielding (4.43) that proves the lemma combined with (4.42).

To derive now Lemma 4.33 from Lemma 4.35, we only have to prove that for all $\nu \in \mathcal{G}_{\text {inv }}(\gamma), e_{\nu}^{+}$exists and is independent of $\gamma$. For such a measure $\nu$, write, using Lemma 4.35

$$
\nu\left(+{ }_{\Lambda}\right)=\int_{\Omega} \frac{e^{-H_{\Lambda}^{\eta}(+)}}{Z_{\Lambda}^{\Phi+}(\eta)} \nu(d \eta) \cong \int_{\Omega} \frac{e^{-H_{\Lambda}^{+}(+)}}{Z_{\Lambda}^{\Phi^{+}}(+)} \nu(d \eta),
$$


where $a_{\Lambda} \cong b_{\Lambda}$ means $\lim _{\Lambda} \frac{1}{|\Lambda|}\left|\log \frac{a_{\Lambda}}{b_{\Lambda}}\right|=0$. Since $\Phi^{+}$is the vacuum potential with vacuum state,$+ H_{\Lambda}^{+}\left(+_{\Lambda}\right)=0$, so that the partition function with vacuum boundary condition coincides with that with free boundary condition, yielding:

$$
\nu\left({ }_{\Lambda}\right)=\frac{1}{Z_{\Lambda}^{\Phi^{+}}(+)}=\frac{1}{Z_{\Lambda}^{\Phi^{+}, \mathrm{f}}}=\left[\sum_{\sigma \in \Omega_{\Lambda}} \exp \left(-\sum_{A \subset \Lambda} \Phi_{A}^{+}(\sigma)\right)\right]^{-1}
$$

and

$$
e_{\nu}^{+}=-\lim _{\Lambda \uparrow \mathbb{Z}^{d}} \frac{1}{|\Lambda|} \log Z_{\Lambda}^{\Phi^{+}}(+)=-\lim _{\Lambda \uparrow \mathbb{Z}^{d}} \frac{1}{|\Lambda|} \log Z_{\Lambda}^{\Phi^{+}, \mathrm{f}}
$$

whenever the limit on the r.h.s. exists. To prove this existence, we adapt the standard proof of the existence of the pressure for UAC potentials based on corridors (see e.g. [64]) to our weaker framework, and base our analysis on the following

Lemma 4.46. The differences of vacuum Hamiltonians taken in nested volumes $\Lambda^{\prime} \subset \Lambda$ with free boundary conditions is at most of the order of the volume annulus between them.

$$
\left|H_{\Lambda}^{\Phi^{+}, \mathrm{f}}(\sigma)-H_{\Lambda^{\prime}}^{\Phi^{+}, \mathrm{f}}(\sigma)\right| \leq C \cdot\left|\Lambda \backslash \Lambda^{\prime}\right|
$$

Proof: This estimate follows as above by choosing an ordering of sites $x$ such that the sites in $\Lambda \backslash \Lambda^{\prime}$ come first. Then we write

$$
\begin{aligned}
\left|H_{\Lambda}^{\Phi^{+}, \mathrm{f}}(\sigma)-H_{\Lambda^{\prime}}^{\Phi^{+}, \mathrm{f}}(\sigma)\right| & =\left|\sum_{\substack{A \subset \Lambda \\
A \cap\left(\Lambda \backslash \Lambda^{\prime}\right) \neq \emptyset}} \Phi_{A}^{+}(\sigma)\right| \\
& =\left|\sum_{x \in \Lambda \backslash \Lambda^{\prime}} \sum_{A \subset \Lambda: A \geq x} \Phi_{A}^{+}(\sigma)\right| \\
& =\left|\sum_{x \in \Lambda \backslash \Lambda^{\prime}} \sum_{A \subset \Lambda: A \geq x} \Phi_{A}^{+}\left(+<x \sigma_{\Lambda \geq x}\right)\right| \\
& \leq C \cdot\left|\Lambda \backslash \Lambda^{\prime}\right|
\end{aligned}
$$

uniformly in $\sigma \in \Omega$, with $C=\sup _{\Lambda, \Lambda^{\prime}, x, \sigma} \cdot\left|H_{\Lambda}^{\Phi^{+}, \mathrm{f}}\left(+_{<x} \sigma_{\Lambda \geq x}\right)\right|<\infty$.

We use this lemma to neglect the contributions of corridors in the partition function and consider volumes $C_{1}, \ldots, C_{n}$, that are lattice translates of each other, whose union is some bigger volume $C=\bigcup_{i} C_{i}$, nested as soon as we assume that $d\left(C_{i}, C_{j}\right) \geq r>0$ for all $i \neq j$. If one considers again an ordering such that the sites in $C_{1}$ come first, one has 


$$
\begin{aligned}
\mid H_{C}^{\Phi^{+}, \mathrm{f}}(\sigma) & -H_{C_{1}}^{\Phi^{+}, \mathrm{f}}(\sigma)-H_{\cup_{i \geq 2}{ }^{+} C_{i}} \mid \\
& =\left|\sum_{x \in C_{1}} \sum_{A \geq x, A \cap \cup_{i \geq 2} C_{i} \neq \emptyset} \Phi_{A}^{+}\left(+<x \sigma_{C \geq x}+C^{c}\right)\right| \\
& =\left|\sum_{x \in C_{1}} \sum_{A \ni x, A \cap B_{r}^{c}\left(C_{1}\right) \neq \emptyset} H_{x}^{\Phi^{+}, \mathrm{f}}\left(+<x \sigma_{C \geq x}+C^{c}\right)\right| \\
& \leq\left|C_{1}\right| \cdot \phi(r)
\end{aligned}
$$

where $B_{r}\left(C_{1}\right)$ is the set of points with distance less or equal to $r$ to $C_{1}$ and $\phi(r)$ goes to zero as $r$ goes to infinity. By induction, one gets

$$
\left|H_{C}^{\Phi^{+}, \mathrm{f}}(\sigma)-\sum_{i} H_{C_{i}}^{\Phi^{+}, \mathrm{f}}(\sigma)\right| \leq n \cdot\left|C_{1}\right| \cdot \phi(r) .
$$

Finally to prove the existence of the limit (4.45) along a sequence of cubes we can now follow the lines of the standard proof [64] which subdivides a large cube of size $n$ into sub-cubes of fixed size $b$, separated by corridors of width $r>0$. We then invoke (4.47) to estimate the contributions of corridors, and (4.50) to estimate the contribution from interactions between different cubes.

\section{Remark 4.51.}

Recently, while these notes were on the point of being achieved, Etienne Mahé has introduced a class of random fields strictly larger than the quasilocal ones, the Asymptotically Sub-Volumic (ASV) measures, whose defining property is the specification counter part of condition $\mathrm{C} 2$, and developed a wide thermodynamical formalism for them [100]. In particular, he proves there that quasilocal measures have this ASV property and combined with our general Theorem 4.24, this provides an alternative proof of 4.32 that relies on specific properties of specifications.

\subsection{More on equilibrium: LDP and Stochas- tic Ising models}

\subsubsection{Large deviation properties}

The thermodynamical variational principle (4.11), valid for translationinvariant Gibbs measures consistent with a translation-invariant potential [52] or for the more general class of asymptotic decoupled measures [104], is a first step toward the statement of a large deviation principle for such measures. Indeed, when relative entropy is defined and can be expressed as 
the convex transform of the pressure, it is often the so-called rate function for such an LDP at the level of measures. We do not enter into the general details of this important subject of probability theory in these lectures, see e.g. $[19,92,93,36]$ for a precise formulation of large deviation principles and their relationships with the notion of entropy in thermodynamics.

Roughly speaking, large deviations consist in a study the probability of rare events by estimating the usually very small probabilities of large simultaneous fluctuations in a system consisting of a large number of random variables. At the level of measures, claiming that such a principle holds is a way of expressing that the probability of a typical configuration from a measure $\mu \in \mathcal{M}_{1 \text {,inv }}^{+}$to look typical in $\Lambda$ for the measure $\nu \in \mathcal{M}_{1, \text { inv }}^{+}$ decays exponentially fast with the volume with a rate equals to the relative entropy $h(\mu \mid \nu)$, that is

$$
\operatorname{Prob}_{\nu}\left[\omega_{\Lambda} \text { typical for } \mu_{\Lambda}\right] \approx e^{-|\Lambda| h(\mu \mid \nu)} .
$$

Thus, when such a principle holds for our Gibbs measures, the rate function gets its minimum at zero when $\mu$ and $\nu$ are Gibbs for the same specification, and the probability of getting from $\mu$ a typical configuration for $\nu$ decays exponentially with the order of the surface only, or at least at a sub-volumic rate. If it does not hold, it implies then that the involved measures cannot be Gibbs for the same potential. ${ }^{13}$

\subsubsection{Stochastic Ising models}

Stochastic Ising models are particular types of Markov Processes on the configuration space $(\Omega, \mathcal{F})$ when the single-site state space is an Ising one $(E=\{-1,+1\})$. They are thoroughly described in [94] and allow, under mild conditions, to get Gibbs measures as invariant reversible measures for these stochastic processes. Let us focus on the most standard local stochastic dynamics, the so-called Glauber or spin-flip dynamics, which corresponds to usual birth and death processes in standard probability theory. Starting from an a priori configuration, one would like to change (or "flip") the configuration at a given site in a random way that favours alignment between neighbors such that the configuration evolves towards a typical configuration of a given Gibbs measure.

To formalize this a bit, one denotes for any $i \in S$ and $\sigma \in \Omega$ the flipped configuration $\sigma^{i}$, defined by $\sigma_{i}^{i}=-\sigma_{i}$ and $\sigma_{j}^{i}=\sigma_{j}$ for all $i \neq j$, and consider a collection of spin-flip rates $\left\{c_{i}(\sigma), i \in S, \sigma \in \Omega\right\}$, assumed to be of finite-range, strictly positive and translation-invariant. They uniquely define a Feller process $\left(\eta_{t}\right)_{t \geq 0}$ on $(\Omega, \mathcal{F})$ with generator ${ }^{14} L$ defined on

\footnotetext{
${ }^{13}$ This is used to detect non-Gibbsianness in the projection of the Ising model [112].

${ }^{14}$ For a rigorous description, consult $[94,35]$.
} 
local functions $f \in \mathcal{F}_{\text {loc }}$ by

$$
L f(\sigma)=\sum_{i \in S} c_{i}(\sigma)\left[f\left(\sigma^{i}\right)-f(\sigma)\right] .
$$

Denoting by $S(t)$ the corresponding semi-group (see [94]) and by $\mathbb{E}_{\sigma}$ the expectation under the corresponding path-space measure $\mathbb{P}_{\sigma}$ given the initial configuration $\eta_{0}=\sigma$, one gets an action on functions $f \in \mathcal{F}_{\text {loc }}$ with for all $t>0$,

$$
(S(t) f)(\sigma)=\mathbb{E}_{\sigma}\left[f\left(\eta_{t}\right)\right]
$$

and on measures $\nu$ to get a time-evolved measure $\nu S(t)$ defined by its expectations on local functions

$$
\int f d(\nu S(t))=\int S(t) f d \nu
$$

This measure $\nu S(t)$ corresponds to the distribution of the configuration at time $t$ when the initial distribution at time zero is $\nu$. A probability measure $\mu \in \mathcal{M}_{1, \text { inv }}(\Omega)$ is then called invariant for the process (or for the dynamics) with generator $L$ iff

$$
\int L f d \mu=0, \forall f \in \mathcal{F}_{\text {loc }}
$$

or equivalently iff $\mu S(t)=\mu$ for all time $t$; an invariant measure $\mu$ is moreover reversible when

$$
\int(L f) g d \mu=0, \forall f, g \in \mathcal{F}_{\text {loc }}
$$

In words, a probability measure is invariant when the process $\left(\eta_{t}\right)_{t}$ obtained by using $\mu$ as initial distribution is stationary in time, so that its definition can be extended to negative times, and is thereafter reversible when the process $\left(\eta_{t}\right)_{t}$ and $\left(\eta_{-t}\right)_{t}$ have the same distribution. In our case, reversibility is equivalent to a standard detailed balance condition on the rates

$$
\frac{d \mu^{i}}{d \mu}=\frac{c_{i}\left(\sigma^{i}\right)}{c_{i}(\sigma)}
$$

where $\mu^{i}$ is the image law of $\mu$ by the spin-flip at site $i, \sigma \longmapsto \sigma^{i}$. Thus, to get a dynamics evolving towards a given measure $\mu$, it is enough to choose the rates according to (4.52). This is the way Gibbs measures are obtained as reversible invariant measures in the so-called Glauber dynamics at inverse temperature $\beta$ : Given a $\mathrm{UAC}$ potential $\Phi$, one introduces the rates

$$
c_{i}(\sigma)=\exp \left\{\frac{\beta}{2} \sum_{A \ni i}\left[\Phi_{A}(\sigma)-\Phi_{A}\left(\sigma^{i}\right)\right]\right\}
$$


in order that (4.52) holds for the Gibbs measures corresponding to the potential $\Phi$. In such a case, the rates generates jump-processes on the configuration space with independent Poisson clocks attached at each site that randomly produce spin-flips according to the considered Gibbs measures, which are eventually the invariant reversible measures reached at equilibrium.

Similar stochastic Ising models can be introduced by changing the spinflip rules (in the so-called Metropolis-Hastings dynamics) or by exchanging the spins between two sites (Kawasaki dynamics, equivalent to an exclusion-process in the lattice gas settings with single site state-space $E=\{0,1\}$ ), leading at equilibrium to the same reversible Gibbs measures $[94]$. 


\section{Chapter 5}

\section{Generalized Gibbs measures}

\subsection{Heuristics}

Let us consider physical systems with a large number of particles in thermal equilibrium modelled by the Gibbsian formalism described in Chapter 3 and consider more precisely the example of particles of water. Although water is too complicated a system to be described precisely by the Gibbsian formalism ${ }^{1}$, it allows to give a qualitative picture of the phase transition phenomenon, in accordance with the precise description of the phase diagram that can be achieved within the Gibbsian formalism by the PirogovSinai theory [106]. The system could be in different states depending on the temperature, and assume that these states are described by extremal Gibbs measures $\mu_{S}, \mu_{L}, \mu_{V}$ at low temperature or $\mu$ at high temperature. One observes the existence of a critical temperature $T_{c}$ that distinguishes a region of temperature where the physical system can only be in a unique phase and a lower dimensional manifold ${ }^{2}$ of non-uniqueness. The latter corresponds to the region in the $(P, T)$-plane where the system can coexist in two or three different states, depending on the pressure for a given temperature, yielding the following (qualitative) phase diagram.

$T>T_{c}:$ Uniqueness regime: $\mathcal{G}(\gamma)=\{\mu\}$.

\footnotetext{
${ }^{1}$ The solid phase has to be more carefully described than here, due to the nature of crystals. One expects that a crystal breaks the translation symmetry, so that translation-invariant Gibbs measures can be ergodic but not extremal in the set of Gibbs measures. Moreover, although there is a gas-liquid point, there is not a second-order transition from solid to liquid or gas in great generality. Our present heuristics has thus to be taken very carefully while dealing with this solid phase.

${ }^{2}$ To get details about these manifolds and the Gibbs phase rule, see Wightman [64].
} 
$T<T_{c}$ : There exist $2 \mathrm{~d}$-manifolds where the system is in a unique phase, solid, liquid or gaseous, depending on the pressure $P$. These uniqueness manifolds have as boundaries 1d-manifolds where two different phases coexists. These so-called coexistence lines have as boundary a 0d-manifold where all three phases coexist, the tricritical point.

From a naive ${ }^{3}$ physical point of view, a phase transition is induced by a transformation of $(P, T)$-variables that allows to go from a region of uniqueness to a region of non-uniqueness. Two kinds of phase transitions are often distinguished: When this $(P, T)$-transformation crosses a coexistence line, one says that a first order phase transition occurs, whereas when one crosses the tricritical point $\mathrm{C}$ from $T>T_{c}$, one often ${ }^{4}$ says that it is second order. Let us focus on the latter, for which a quantity called correlation length is introduced. Consider as usual a Gibbs measure $\mu$ and a configuration $\sigma \in \Omega$. For $T \neq T_{c}$, and $i \neq j \in S$, the covariance between the random variables $\sigma_{i}$ and $\sigma_{j}$ is expected ${ }^{5}$ to decay exponentially in such a way that one introduces a quantity $\xi(T)$ such that:

$$
\mu\left(\sigma_{i} \sigma_{j}\right)-\mu\left(\sigma_{i}\right) \mu\left(\sigma_{j}\right) \cong e^{-\frac{|i-j|}{\xi(T)}}
$$

where $\cong$ means logarithmic equivalence for large $|i-j|$. The quantity $\xi(T)$ has the dimension of a distance and is interpreted as the correlation length of the system, beyond which two spins are physically considered to be independent. This cannot be done at the critical temperature because the decay of correlation is not expected to be exponential and in some sense one has presumably

$$
\lim _{T \rightarrow T_{c}} \xi(T)=+\infty
$$

This is interpreted as the absence of proper scale for the system at the critical temperature. Physically, for the system of water, we observe a "milky" water or critical opalescence, due to the appearance of fluctuations of all orders, including in particular very large ones ${ }^{6}$ that creates a

\footnotetext{
${ }^{3}$ There actually exists too main complementary notions of phase transition: A thermodynamic notion corresponding to the lack of analyticity of a thermodynamical function (free energy, pressure, etc.) and a statistical mechanical one corresponding to a change in the number of extremal states.

${ }^{4}$ Crossing a critical point does not automatically imply a second order phase transition. For instance, its crossing in the $1 / r^{2}$-Ising model in one dimension is of first order with a jump in the magnetization. The order is more precisely related to the presence of various critical phenomena (divergence of the correlation length, fluctuations at all scales, etc.) or to the order of the diverging derivative of the free energy, see $[24,36,54,66,113]$. This distinction between first or second order phase transitions is also mathematically characterized in terms of differentiable properties of the pressure introduced in Chapter 4, see [64, 113, 36].

${ }^{5} \mathrm{It}$ is sometimes proved for standard models, see e.g. [87].

${ }^{6}$ As these fluctuations occur also in a scale of the order of visible light, a scattering phenomenon occurs that yields the whitish color.
} 
highly chaotic behavior. As a consequence of this absence of proper scale, the behavior of the system at the critical point should be the same at any scale, providing a tool to study these ill-known critical behaviors: Natural transformations of the observation scale seem to be an appropriate tool to understand it better, the critical point being considered in some sense as a fixed point of these transformations. This has motivated the introduction, as a tool in theoretical physics to study critical phenomena, of a semi-group of transformations directly related to a change of scale of the system, the Renormalization Group, that appeared to be rather powerful in these fields, see e.g. [17, 24, 48, 50, 56, 119].

We shall describe it more precisely in the next section, but let us first consider a scaling transformation $T: \Omega \longrightarrow \Omega^{\prime}$, where $\Omega$ and $\Omega^{\prime}$ are the configuration spaces at two different scales. Let $\mu$ be a measure describing an equilibrium state of the system at the first scale, i.e. a Gibbs measure on $\Omega$, and denote formally $H$ its Hamiltonian. The transformation $T$ acts naturally on measures and we denote $\mu^{\prime}=T \mu \in \mathcal{M}_{1}^{+}\left(\Omega^{\prime}, \mathcal{F}^{\prime}\right)$. The natural aim in our theory would be to obtain $\mu^{\prime}$ as a Gibbs measure and to define an Hamiltonian $H^{\prime}$, image of the Hamiltonian $H$ by a renormalization transformation on spaces of Hamiltonians for it, in order to get the following diagram well defined and commutative.

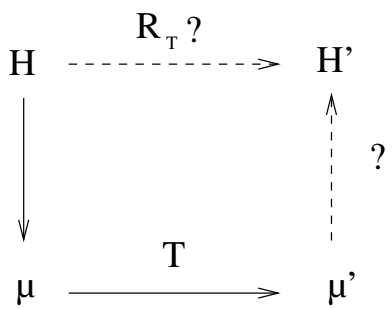

Figure 1: A renormalization group transformation acting on measures.

In the late seventies and early eighties, Griffiths and Pearce/Israel [56, 63] discovered some pathologies of the behavior of these image measures: It turned out that they often are not Gibbsian. It came out as a surprise that one could break the equilibrium properties of a state by only looking at it at a different scale, and according to the ideology of the RG theory, this should not be so. These pathologies have been rigorously proven to exist and mostly identified as the manifestation of non-Gibbsianness due to a failure of the quasilocality property in 1993 by van Enter, Fernández and Sokal [36] in a rather general and rigorous mathematical framework. Let us recall that the Hamiltonian can be recovered from the Gibbs measure $\mu$ with the help of the Moebius inversion formula. When the correlations do 
not decrease sufficiently fast, and in particular at the critical point or in some phase transitions region, a divergence might appear in this inversion, preventing a definition of $H^{\prime}$ from $\mu^{\prime}$, and leading to non-Gibbsianness of the image-measure. Nevertheless, we shall see that this phenomenon also holds in other parts of the phase diagram, sometimes far from the critical point, and that it is already present after one single change of scale for very simple scaling transformations.

\subsection{RG pathologies and non-Gibbsianness}

In this section, we formally introduce the renormalization group transformations and describe how these transformations could lead to nonGibbsianness. As we shall see, this phenomenon is often related to the occurrence of phase transitions in some hidden or constrained system, and to get the main features of the phenomenon we describe precisely how this happens for the simplest RG transformation, the so-called decimation of the $2 d$ Ising model at low temperature, the latter being low enough to get a phase transition that creates long-range dependencies leading to nonquasilocality in the mentioned hidden system. Thereafter, we shall give a (non-exhaustive) catalogue of other RG transformations of Gibbs measures that also lead to non-Gibsianness for similar reasons, but for which the proof is much more complicated, when it exists.

\subsubsection{Decimation of the $2 d$ ferromagnetic Ising model}

The basic example we describe here, which already captures the main nontrivial features of the pathologies, concerns the decimation of the standard $2 d$-Ising model, or more precisely the projection of the $2 \mathrm{~d}$-Ising model on the sublattice of even sites [36]. We shall give similar results for decimations at other dimensions and for other spacings later on. At a general level, the decimation transformation on $\mathbb{Z}^{2}$ with spacing $b$ is defined to be the transformation

$$
\begin{aligned}
T_{b}:(\Omega, \mathcal{F}) & \longrightarrow\left(\Omega^{\prime}, \mathcal{F}^{\prime}\right)=(\Omega, \mathcal{F}) \\
\omega & \longmapsto \omega^{\prime}=\left(\omega_{i}^{\prime}\right)_{i \in \mathbb{Z}^{2}}
\end{aligned}
$$

defined by $\forall i \in \mathbb{Z}^{2}$ by $\omega_{i}^{\prime}=\omega_{b i}$. We denote simply $T=T_{2}$ the case of a spacing $b=2$ described here. The following result is crucial to understand RG pathologies and the arising of non-Gibbsianness in equilibrium mathematical statistical mechanics. This example, already introduced by Israel in [63] to detect RG pathologies, has been fully analyzed in [36], which constitutes the seminal description of $\mathrm{RG}$ pathologies in the realm of Gibbsianness vs. non-Gibbsianness framework. 
Theorem 5.1. [36] Let $\beta>\tilde{\beta}_{c}=\frac{1}{2} \cosh ^{-1}\left(e^{2 \beta_{c}}\right)$ and denote by $\nu_{\beta}=T \mu_{\beta}$ the decimation of any Gibbs measure $\mu$ for the homogeneous ferromagnetic n.n Ising model on $\mathbb{Z}^{2}$ with zero magnetic field. Then $\nu_{\beta}$ is not quasilocal, hence non-Gibbs.

To prove non-quasilocality of the renormalized measure, one exhibits a so-called bad configuration where the conditional expectation of a local function (w.r.t. the outside of a finite set) is essentially discontinuous, or equivalently is discontinuous on a (non-negligible) neighborhood, as described in Chapter 3. The role of a bad configuration is played by the alternating configuration $\omega^{\text {alt }}$ defined for all $i=\left(i_{1}, i_{2}\right) \in \mathbb{Z}^{2}$ by $\omega_{i}^{\text {alt }}=(-1)^{i_{1}+i_{2}}$. Computing the magnetization under the image measure $\nu_{\beta}$, conditioned on the boundary condition $\omega^{\text {alt }}$ outside the origin, will yield different limits when one approaches this configuration with all + (resp. all -) arbitrarily far away, as soon as phase transition is possible for the Ising model on the so-called decorated lattice, a version of $\mathbb{Z}^{2}$ where even sites have been removed. The latter phase transition is shown to be possible as soon as the inverse temperature is larger than the above value $\tilde{\beta}_{c}$. The global neutrality of this bad configuration leaves the door open to such a phase transition to occur at low enough temperature, and is crucial in its badness. Let us formalize this a bit more, following the full proof given in [36].

Proof of theorem 5.1: Denote by $\nu=T \mu$ this decimated measure:

$$
\forall A^{\prime} \in \mathcal{F}^{\prime}, \nu\left(A^{\prime}\right)=\mu\left(T^{-1}\left(A^{\prime}\right)\right)=\mu(A)
$$

with the notation $A=T^{-1}\left(A^{\prime}\right) \in \mathcal{F}$. In order to describe how a phase transition in some hidden system gives rise to non-Gibbsianness, we also extend this decimation on the "even" sites of $\mathbb{Z}^{2}$, i.e. $2 \mathbb{Z}^{2}$, by:

$$
T: i=2 i^{\prime} \longmapsto i^{\prime}
$$

and on subsets: $\forall \Lambda \subset 2 \mathbb{Z}^{2}, T(\Lambda):=\Lambda^{\prime}=\left\{i \in \mathbb{Z}^{2}, 2 i \in \Lambda\right\} \subset \mathbb{Z}^{2}$. We underline that $T$ maps the finite subsets of $2 \mathbb{Z}^{2}$ on the finite subsets of $\mathbb{Z}^{2}$, but the converse is not true: If one defines a cofinite subset of $S$ to be the complement of any finite set, then the inverse transformation $T^{-1}$ does not map the cofinite subsets of $\mathbb{Z}^{2}$ on the cofinite subsets of $2 \mathbb{Z}^{2}$. For example, when $\Lambda^{\prime}=\{\mathbf{0}\}$ consists of the origin $\mathbf{0}$ of $\mathbb{Z}^{2}$. Then $\Lambda^{\prime c}=\mathbb{Z}^{2} \backslash\{\mathbf{0}\}$ 


$$
\text { and } \begin{aligned}
T^{-1}\left(\Lambda^{\prime c}\right) & =\left\{i=2 i^{\prime} \text { s.t. } i^{\prime} \in \Lambda^{\prime c}\right\}=\left\{x=2 x^{\prime}, x^{\prime} \in \mathbb{Z}^{2}, x^{\prime} \neq 0\right\} \\
& =2 \mathbb{Z}^{2} \backslash\{\mathbf{0}\}=\left[\left(\mathbb{Z}^{2} \backslash 2 \mathbb{Z}^{2}\right) \cup\{\mathbf{0}\}\right]^{c}=: \lambda^{c}
\end{aligned}
$$

where $\lambda=\left(\mathbb{Z}^{2} \backslash 2 \mathbb{Z}^{2}\right) \cup\{\mathbf{0}\}$ is not a finite subset of $\mathbb{Z}^{2}$.

This is actually the reason why non-Gibbsianness could occur: Getting still an infinite-volume framework after this conditioning, this leaves the possibility of phase transitions for the measure conditioned on $\lambda$, which in turn would give rise to the essential discontinuity. In order to prove that $\nu$ is not Gibbsian, we prove that there exists $\Lambda^{\prime} \in \mathcal{S}^{\prime}$ and a function $f$ local on $\Omega^{\prime}$ such that no version of $\nu\left[f \mid \mathcal{F}_{\Lambda^{\prime c}}\right](\cdot)$ is quasilocal, or equivalently we want to find $\omega^{\prime}$ in $\Omega^{\prime}$ for which there exists $f$ local on $\Omega^{\prime}$ with $\nu\left[f \mid \mathcal{F}_{\Lambda^{\prime c}}\right]\left(\omega^{\prime}\right)$ essentially discontinuous. Now, using the action of the measurable map $T$ on subsets and configurations, one easily gets, for any $\Lambda^{\prime} \in \mathcal{S}^{\prime}$

$$
\nu\left[A^{\prime} \mid \mathcal{F}_{\Lambda^{\prime c}}^{\prime}\right]\left(\omega^{\prime}\right)=\mu\left[A \mid \mathcal{F}_{\lambda^{c}}\right]\left(T^{-1} \omega^{\prime}\right), \nu \text {-a.e. }\left(\omega^{\prime}\right), \forall A^{\prime} \in \mathcal{F}^{\prime} .
$$

So we have to compute the conditional probabilities $\mu\left[A \mid \mathcal{F}_{\lambda^{c}}\right]$ for $\lambda$ nonfinite, and this is not given by the specification $\gamma$, which only provides versions of conditional probabilities for the outside of finite sets only ${ }^{7}$. Thus $\lambda=T^{-1}\left(\Lambda^{\prime c}\right)$ is not a cofinite set, as we show for $\Lambda^{\prime}=\{\mathbf{0}\}$ and illustrated on the figures 2 and $2 \mathrm{~b}$ below:

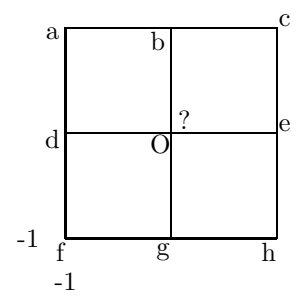

Figure 2: The configuration space after decimation, $\Omega^{\prime}$.

\footnotetext{
${ }^{7}$ To describe such conditional probabilities, on should use global specifications developed in [44].
} 
A short computation leads indeed to

$$
\lambda^{c}=\mathbb{Z}^{2} \backslash\left(2 \Lambda^{\prime}\right)=T^{-1}\left(\Lambda^{\prime}\right) \cup\left(\mathbb{Z}^{2} \backslash 2 \mathbb{Z}^{2}\right)
$$

and thus $\lambda^{c}$ consists of all the spins of $2 \mathbb{Z}^{2}$ except the origin: If we "knew" everything except the origin on the decimated system $\Omega$, we should "know" the spins on $2 \mathbb{Z}^{2}$ except at the origin. Then $\lambda$ is the origin plus the sites which are not in $2 \mathbb{Z}^{2}$, as shown below ${ }^{8}$

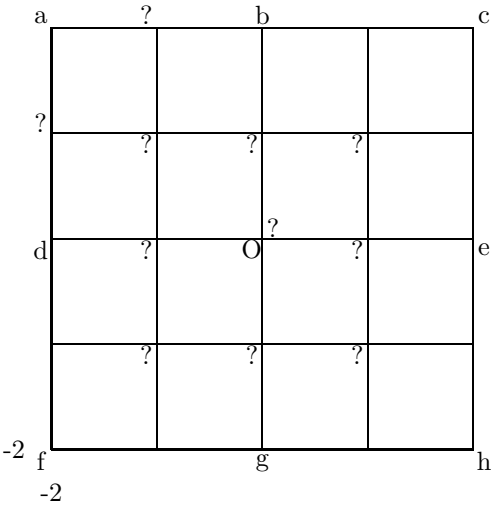

Figure $2 \mathrm{~b}$ : The configuration space before decimation, $\Omega$.

To prove the failure of quasilocality, we thus have to compute $\mu\left[\cdot \mid \mathcal{F}_{\lambda^{c}}\right](\omega)$ when $\omega \in T^{-1}\left(\omega^{\prime}\right)$, with $\omega^{\prime}$ in the neighborhood of a particular configuration in $\Omega^{\prime}$. Of course, we know that $\mu$ is a Gibbs measure for the $2 d$-Ising model, so there exists $\Omega_{\mu}$ with $\mu\left(\Omega_{\mu}\right)=1$ s.t. for all $\omega \in \Omega_{\mu}$, for all $\sigma \in \Omega$ and $\Lambda \in \mathcal{S}$,

$$
\mu\left[\sigma \mid \mathcal{F}_{\Lambda^{c}}\right](\omega)=\frac{1}{\mathbf{Z}_{\Lambda}(\omega)} \exp \left(\sum_{\langle i j\rangle \subset \Lambda} \beta \sigma_{i} \sigma_{j}+\sum_{\langle i j\rangle, i \in \Lambda, j \in \Lambda^{c}} \beta \sigma_{i} \omega_{j}\right)
$$

but we want to study $\mu\left[\cdot \mid \mathcal{F}_{\lambda^{c}}\right]$ with $\lambda$ non-finite, which is not a finitevolume probability, but on the contrary appears to be an infinite-volume Gibbs measure, as generally proved in [36]:

Lemma 5.3. [36] Let $\omega^{\prime} \in \Omega^{\prime}$ and let $\lambda$ a infinite subset of $\mathbb{Z}^{2}$. Then the restriction of $\mu\left[\cdot \mid \mathcal{F}_{\lambda^{c}}\right]\left(\omega^{\prime}\right)$ to $\left(\Omega_{\lambda}, \mathcal{F}_{\lambda}\right)$ is a Gibbs measure for a UAC potential $\Phi=\Phi\left(\lambda, \omega^{\prime}\right)$.

\footnotetext{
${ }^{8}$ The letters denote the value of spins on the underlying sites, when fixed, and the ? indicate that the spin over the underlying site is unknown.
} 
We shall not prove this lemma in the general case, but rather directly establish the result for a particularly well-chosen configuration, for which a phase transition is possible for the resulting Gibbs measure on the smaller infinite-volume configuration space $\left(\Omega_{\lambda}, \mathcal{F}_{\lambda}\right)$. This particular configuration will now be in some neighborhood $\mathcal{N}_{\Lambda}$ of the (neutral) alternating configuration $\omega^{\text {alt }}$ defined by

$$
\forall i=\left(i_{1}, i_{2}\right) \in \mathbb{Z}^{2}, \omega_{i}^{\prime \text { alt }}=(-1)^{i_{1}+i_{2}} .
$$

Denote $\mu^{\omega^{\prime}, \lambda}$ the restriction of $\mu\left[\cdot \mid \mathcal{F}_{\lambda^{c}}\right]\left(\omega^{\prime}\right)$ to $\left(\Omega_{\lambda}, \mathcal{F}_{\lambda}\right)$, well-defined as a probability measure by the existence of regular versions of conditional probabilities as described in Chapter 3. As $\lambda$ is fixed (we always take now $\Lambda^{\prime}=\{\mathbf{0}\}$ ), we forget it and write $\mu^{\omega^{\prime}, \lambda}=\mu^{\omega^{\prime}}$. To prove that it is a Gibbs measure on $\left(\Omega_{\lambda}, \mathcal{F}_{\lambda}\right)$, we consider $\Delta \subset \lambda$ finite and a boundary condition $\tau \in \Omega_{\lambda}$, which yields the following picture:

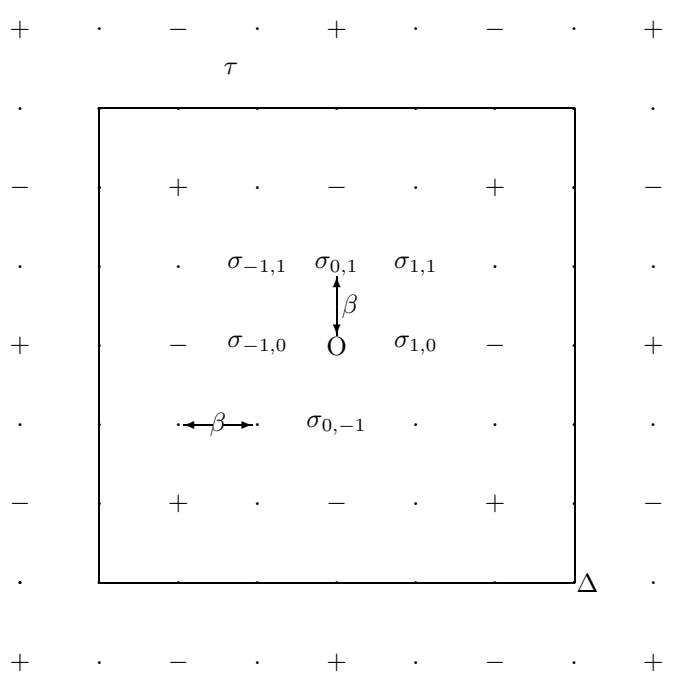

Figure 3: Configuration space $\Omega_{\lambda}$ with $\omega^{\text {alt }}$ in $\lambda^{c}$.

To check the D.L.R. equations for a suitable interaction, we have to compute, for $\omega^{\prime}$ in the neighborhood $\mathcal{N}_{\Lambda}$ of $\omega^{\text {alt }}$, for $\mu^{\omega^{\prime}}$-a.e. $\tau$ and for all $\sigma_{\lambda} \in \Omega_{\lambda}$

$$
\left.\mu^{\omega^{\prime}}\left[\sigma \mid \mathcal{F}_{\lambda \backslash \Delta}\right](\tau)=\tau_{\lambda \backslash \Delta}\right]=\sum_{\sigma_{\lambda^{c}} \in \Omega_{\lambda^{c}}} \mu\left[\sigma \mid \sigma_{\lambda \backslash \Delta}=\tau_{\lambda \backslash \Delta}, \sigma_{\lambda^{c}}=\omega_{\lambda^{c}}\right]
$$


with $\omega \in T^{-1}\left(\omega^{\prime}\right)$. We first assume that $\Lambda$ is big enough to contain the $\Delta$ considered, in order to describe the resulting interaction. Later on, we shall take the infinite volume for $\Delta$ and eventually encounter a volume where $\omega^{\prime}$ is different to the alternating configuration, to eventually select different phases when possible. For the moment the large $\Lambda$ allows us to work with the single $\omega^{\text {alt }}$ only but also to prevent possible conditioning with sets of measure zero. In the previous sum, only one term is not zero, when $\sigma_{\lambda^{c}}=\omega_{\lambda^{c}}$, which is the alternating configuration on $\lambda^{c}$. Hence, using the definition of conditional probability as a probability kernel,

$$
\mu^{\omega^{\prime}}\left[\sigma_{\lambda} \mid \sigma_{\lambda \backslash \Delta}=\tau_{\lambda \backslash \Delta}\right]=\mu\left[\sigma \mid \mathcal{F}_{\Delta^{c} \cup \lambda^{c}}\right]\left(\tau_{\lambda} \omega_{\lambda^{c}}\right) .
$$

But $\Delta^{c} \cup \lambda^{c}=(\Delta \cap \lambda)^{c}$, and $\Delta \cap \lambda=\Delta$ is a finite subset of $\mathbb{Z}^{2}$, so we now use the D.L.R. equations for $\mu$ to get, for $\mu$-a.e. $\tau_{\lambda} \omega_{\lambda^{c}} \in \Omega$ :

$$
\begin{gathered}
\mu\left[\sigma_{\lambda} \mid \sigma_{\Delta^{c}}=\tau_{\Delta^{c}}\right]= \\
\frac{1}{\mathbf{Z}_{\Delta}^{\omega^{\prime}(\tau)}} \exp \left(\beta\left(\sum_{\langle i j\rangle \subset \Delta} \sigma_{i} \sigma_{j}+\sum_{\langle i j\rangle, i \in \Delta, j \in \lambda^{c}} \sigma_{i} \omega_{j}+\sum_{\langle i j\rangle, i \in \Delta, j \in \lambda \cap \Delta^{c}} \sigma_{i} \tau_{j}\right)\right)
\end{gathered}
$$

where the normalization is the standard partition function. A remarkable fact now, that will eventually lead us to consider the Ising model on the decorated lattice, is that in the sum $\sum_{\langle i j\rangle, i \in \Delta, j \in \lambda^{c}} J \sigma_{i} \omega_{j}$, the $j$ 's are "even", i.e. $j=2 k$ with $k \in \mathbb{Z}^{2}$ such that $\omega_{j}=\omega_{k}^{\prime}$ is fixed in the alternating configuration. Then, we obtain the validity of the DLR equation for $\mu^{\omega^{\prime}}$-almost $\tau \in \Omega_{\lambda}$, i.e we have proved the :

Lemma 5.4. Let $\omega^{\prime}$ be the alternating configuration and assume that there exists $\omega \in T^{-1}\left(\omega^{\prime}\right)$ for which the D.L.R. equations for $\mu$ are valid. Then $\mu^{\omega^{\prime}}$, the restriction of $\mu\left[\cdot \mid \mathcal{F}_{\lambda^{c}}\right](\omega)$ on $\left(\Omega_{\lambda}, \mathcal{F}_{\lambda}\right)$ is a Gibbs measure for some UAC potential.

We do not need to give explicitly the potential in general, but it appears to be equivalent to an Ising potential on the decorated lattice in the the computation of the magnetization that we perform now. We shall then observe (Figure 4) that the coupling due to "even" sites cancels and we obtain a Gibbs measure for an Ising model on $\left(\Omega_{\lambda}, \mathcal{F}_{\lambda}\right)$, with the same definition of the nearest-neighbors as in $\mathbb{Z}^{2}$. To achieve this, we just need to know that there is some Gibbs measure for the interaction of the previous equation. In case of phase transition, we do not know which phase it could be, and we shall prove that local variations in $\omega^{\prime}$ could change drastically the selected one. This will yield the non-Gibbsianness of the decimated measure. 


\section{Computation of the magnetization}

To prove a non quasilocality of $\nu$ at sufficiently low temperature, consider then the action of the conditional probabilities on a local function chosen to be characteristic of the phase transition mentioned above. Namely, it should be an order parameter of the phase transition ${ }^{9}$ and consider here the so-called magnetization, which reduces to the expectation at the origin for this translation-invariant model. To do so, we denote again its origin by $\mathbf{0}$ or $(0,0)$ and consider the local function $f: \Omega^{\prime} \longrightarrow \mathbb{R} ; \sigma^{\prime} \longmapsto$ $f\left(\sigma^{\prime}\right)=\sigma_{\mathbf{0}}^{\prime}$. We study $\nu\left[\sigma_{\mathbf{0}}^{\prime} \mid \mathcal{F}_{\Lambda^{\prime c}}\right]\left(\omega^{\prime}\right)$ for $\omega^{\prime}$ in the neighborhood of the alternating configuration, considering first that $\Lambda$ is big enough to feel $\omega^{\prime}$ as the alternating configuration itself. Then, $\nu$-a.s.

$$
\nu\left[\sigma_{0}^{\prime} \mid \mathcal{F}_{\Lambda^{\prime c}}\right]\left(\omega^{\prime}\right)=\mu^{\omega^{\prime}}\left[\sigma_{0}\right]
$$

as described in the previous section. We know that it is a Gibbs measure for some interaction, then by Lemma 2.65 there exists a sequence $\left(\nu_{R} \gamma_{\Lambda_{R}}\right)_{R \in \mathbb{N}}$ whose weak limit is $\mu^{\omega^{\prime}}$. For $R \in \mathbb{N}$, write, by a slight abuse of notation, $\Lambda_{R}$ for the intersection between $\lambda$ and the usual cube of length $2 R$ s.t. there exists a sequence $\nu_{R}$ with

$$
\left\langle\sigma_{\mathbf{0}}\right\rangle^{\omega^{\prime}}:=\mu^{\omega^{\prime}}\left[\sigma_{\mathbf{0}}\right]=\lim _{R \rightarrow \infty}\left\langle\sigma_{\mathbf{0}}\right\rangle^{\omega^{\prime}, \nu_{R}},
$$

where

$$
\left\langle\sigma_{\mathbf{0}}\right\rangle^{\omega^{\prime}, \nu_{R}}:=\int_{\Omega} \mu^{\omega^{\prime}}\left[\sigma_{\mathbf{0}} \mid \mathcal{F}_{\Lambda_{R}^{c}}\right]\left(\tau_{R}\right) d \nu_{R}\left[\tau_{R}\right]
$$

is the expectation of the spin at the origin when the boundary condition which selects $\mu^{\omega^{\prime}}$ has the law $\nu_{R}$. Let us first fix one boundary condition $\tau_{R}$ and note $\langle\cdot\rangle^{\omega^{\prime}, \tau_{R}}$ the expectation under the measure $\mu^{\omega^{\prime}}\left[\cdot \mid \mathcal{F}_{\Lambda_{R}^{c}}\right]\left(\tau_{R}\right)$. We know that $\mu^{\omega^{\prime}}$ is a Gibbs measure on $\left(\Omega_{\lambda}, \mathcal{F}_{\lambda}\right)$, whose lattice consists of all the non-even spins plus the origin.

In order to study this measure on a more conventional lattice, let us try to fix the spin at the origin. Define $L_{R}=\left\{i \in \Lambda_{R}\right.$ s.t. $i_{1}$ and $i_{2}$ are both odd $\}$ and $H_{R}=\Lambda_{R} \backslash L_{R}$. We have, using the notation $\kappa_{R}^{\lambda}\left(d \sigma_{\lambda}\right)$ to denote the product measure $\rho_{\Lambda_{R}} \otimes \delta_{\tau_{\lambda \backslash \Lambda_{R}}}^{\otimes \lambda \backslash \Lambda_{R}}\left(d \sigma_{\lambda}\right)$,

$$
\begin{aligned}
\left\langle\sigma_{\mathbf{0}}\right\rangle^{\omega^{\prime}, \tau_{R}} & =\frac{1}{\mathbf{Z}^{\omega^{\prime}, \tau_{R}}} \int_{\Omega_{\lambda}} \sigma_{\mathbf{0}} e^{\beta\left(\sigma_{\mathbf{0}}-1\right)\left(\sum_{\langle i \mathbf{0}\rangle} \sigma_{i}\right)} e^{\sum_{\langle i j\rangle, i \in \Lambda_{R}, j \in \lambda \backslash \Lambda_{R}} \beta \sigma_{i} \tau_{j}} \\
& \times \prod_{a \in L_{R}}\left(e^{\sum_{\langle i a\rangle \subset \Lambda_{R}} \beta \sigma_{a} \sigma_{i}}\right) \kappa_{R}^{\lambda}\left(d \sigma_{\lambda}\right)
\end{aligned}
$$

\footnotetext{
${ }^{9}$ In statistical mechanics, an order parameter of a UAC potential which admit a family $\left\{\mu_{j}, j \in J\right\}$ of distinct Gibbs measures is a finite system $\left\{f_{1}, \ldots, f_{n}\right\}$ of local functions which discriminate these Gibbs measures by means of the associated expectations $\left\{\mu_{j}\left[f_{1}\right], \ldots, \mu_{j}\left[f_{n}\right]\right\}[52]$.
} 
where $\sum_{\langle i \mathbf{0}\rangle}$ means that the sum is taken over all the spins attached to the origin and $\mathbf{Z}^{\omega^{\prime}, \tau_{R}}$ is a standard normalization. Using Fubini's theorem for positive functions, we first integrate out w.r.t. the origin to get (with $\lambda^{\star}=\lambda$ and $\left.\Lambda_{R}^{\star}=\Lambda_{R} \backslash\{\mathbf{0}\}\right)$

$$
\begin{aligned}
\left\langle\sigma_{\mathbf{0}}\right\rangle^{\omega^{\prime}, \tau_{R}} & =\frac{1}{\mathbf{Z}^{\omega^{\prime}, \tau_{R}}}\left(1-\int_{\Omega_{\lambda^{\star}}} e^{-2 \beta\left(\sum_{\langle i \mathbf{0}\rangle} \sigma_{i}\right)} e^{\sum_{\langle i j\rangle, i \in \Lambda_{R}, j \in \lambda^{\star} \backslash \Lambda_{R}} \beta \sigma_{i} \tau_{j}}\right. \\
& \left.\times \prod_{a \in L_{R}}\left(e^{\sum_{\langle i a\rangle \subset \Lambda_{R}} \beta \sigma_{a} \sigma_{i}}\right) \kappa_{R}^{\lambda^{\star}}\left(d \sigma_{\lambda^{\star}}\right)\right)
\end{aligned}
$$

with the partition function

$$
\begin{aligned}
\mathbf{Z}^{\omega^{\prime}, \tau_{R}} & =1+\int_{\Omega_{\lambda^{\star}}} e^{-2 \beta\left(\sum_{\langle i \mathbf{0}\rangle} \sigma_{i}\right)} e^{\sum_{\langle i j\rangle, i \in \Lambda_{R}, j \in \lambda \backslash \Lambda_{R}} \beta \sigma_{i} \tau_{j}} \\
& \times \prod_{a \in L_{R}}\left(e^{\sum_{\langle i a\rangle \subset \Lambda_{R}} \beta \sigma_{a} \sigma_{i}}\right) \kappa_{R}^{\lambda^{\star}}\left(d \sigma_{\lambda^{\star}}\right) .
\end{aligned}
$$

Hence, we only have to compute the expectation of $e^{-2 \beta\left(\sum_{\langle i \mathbf{0}\rangle} \sigma_{i}\right)}$ w.r.t. the Gibbs distribution with boundary condition $\tau_{R}$ for an Ising model on $\left(\Omega_{\lambda^{\star}}, \mathcal{F}_{\lambda^{\star}}\right)$ when the spin is fixed to be "+" at the origin. We obtain this model because of the very particular interaction we get with the alternating configuration: The contributions of the "even sites", which are fixed in the alternating configuration, cancel each other. We have then the alternating configuration everywhere on $2 \mathbb{Z}^{2}$ and an Ising distribution on the so-called decorated lattice $\lambda^{\star}$, without external magnetic field as soon as $\Delta \subset \Lambda$. Denote $\mu^{+, \omega^{\prime}, \tau_{R}}$ this measure and $\langle\cdot\rangle^{+, \omega^{\prime}, \tau_{R}}$ the expectation with respect to it, to get

$$
\left\langle\sigma_{\mathbf{0}}\right\rangle^{\omega^{\prime}, \tau_{R}}=\frac{1-\left\langle e^{-2 \beta\left(\sigma_{0,1}+\sigma_{1,0}+\sigma_{-1,0}+\sigma_{0,-1}\right)}\right\rangle^{+, \omega^{\prime}, \tau_{R}}}{1+\left\langle e^{-2 \beta\left(\sigma_{0,1}+\sigma_{1,0}+\sigma_{-1,0}+\sigma_{0,-1}\right)}\right\rangle^{+, \omega^{\prime}, \tau_{R}}} .
$$

To get an expression in terms of standard Ising models, we now use the following trick, standard in statistical mechanics with \pm 1 Ising spins, to reduce $\left\langle\sigma_{0,1}\right\rangle^{+, \omega^{\prime}, \tau_{R}}$, the expectation of one spin attached to the origin:

$$
\begin{aligned}
\left\langle\sigma_{0,1}\right\rangle^{+, \omega^{\prime}, \tau_{R}} & =\left\langle\tanh \left(J\left(\sigma_{1,1}+\sigma_{-1,1}\right)\right)\right\rangle^{+, \omega^{\prime}, \tau_{R}} \\
& =\left\langle\left(\frac{1}{2} \tanh (2 J)\right)\left(\sigma_{1,1}+\sigma_{-1,1}\right)\right\rangle^{+, \omega^{\prime}, \tau_{R}}
\end{aligned}
$$

where $\sigma_{1,1}$ and $\sigma_{-1,1}$ are the spins attached to $\sigma_{0,1}$. This reduces our study to the distribution of the spins in $L_{R}$, that is in fact the decorated lattice, the lattice of spins whose coordinates are both odd. 


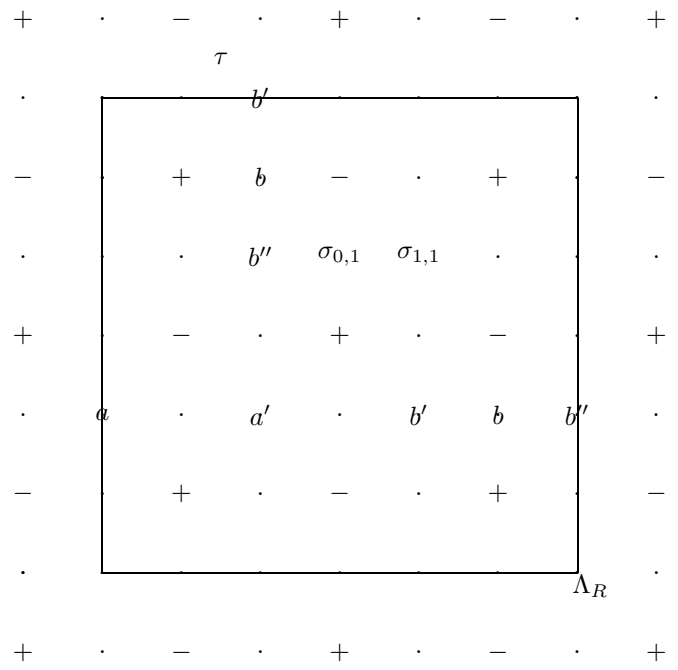

Figure 4 : Ising model on the decorated lattice $\lambda^{*}$

We then have to compute $\left\langle\sigma_{1,1}\right\rangle^{+, \omega^{\prime} \tau_{R}}$. As claimed before, we can start by integrating first with respect to the spins in $H_{R}$, the sites of the decorated lattice which have exactly two neighbors. We call $H_{R}^{0}=H_{R} \backslash \Gamma_{R}$ where $\Gamma_{R}=\Lambda_{R} \backslash \Lambda_{R-1}$ is the boundary of $\Lambda_{R}$. The sites in $H_{R}^{0}$ are those which have two neighbors in $\Lambda_{R}$. We also call $H_{R}^{1}=H_{R} \cap \Gamma_{R}$ the set of the sites which have two neighbors in the lattice $\lambda^{\star}$, one in $\Lambda_{R}$ and the other, fixed by the boundary condition $\tau$, outside $\Lambda_{R}$. Compute:

$\left\langle\sigma_{1,1}\right\rangle^{+, \omega^{\prime} \tau_{R}}=\frac{1}{\mathbf{Z}^{+, \omega^{\prime}, \tau_{R}}} \int_{\Omega_{\lambda^{\star}}} \sigma_{1,1} \cdot A_{R}^{0}\left(\sigma, d \sigma_{H_{R}^{0}}\right) \cdot A_{R}^{1}\left(\sigma, d \sigma_{H_{R}^{1}}\right) \cdot A_{R}\left(\sigma, d \sigma_{L_{R}}\right)$,

with

$$
\begin{aligned}
& A_{R}^{0}\left(\sigma, d \sigma_{H_{R}^{0}}\right)=\prod_{b \in H_{R}^{o}} e^{\beta \sigma_{b}\left(\sigma_{b^{\prime}}+\sigma_{b^{\prime \prime}}\right)} \rho_{0}\left(d \sigma_{b}\right) \\
& A_{R}^{1}\left(\sigma, d \sigma_{H_{R}^{1}}\right)=\prod_{b \in H_{R}^{1}} e^{\beta \sigma_{b}\left(\sigma_{b^{\prime}}+\tau_{b^{\prime \prime}}\right)} \rho_{0}\left(d \sigma_{b}\right)
\end{aligned}
$$

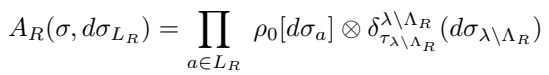

where for each $b \in H_{R}$, we have called $b^{\prime}$ and $b^{\prime \prime}$ its neighbors in $L_{R}$ or 
filled by the boundary condition $\tau$ in $\Lambda_{R+1}$, to get for the integral (5.5)

$$
\int_{\Omega_{L_{R}}} \sigma_{1,1}\left(\prod_{b \in H_{R}} \int_{E} e^{\beta \sigma_{b}\left(\sigma_{b^{\prime}}+\sigma_{b^{\prime \prime}}\right)} \rho_{0}\left[d \sigma_{b}\right]\right) \prod_{a \in L_{R}} \rho_{0}\left[d \sigma_{a}\right] \otimes \delta_{\tau_{\lambda \backslash \Lambda_{R}}}^{\otimes \lambda \backslash \Lambda_{R}}\left[d \tau_{\lambda \backslash \Lambda_{R}}\right] .
$$

Now, using another standard trick on Ising spins, we compute

$$
\int_{E} e^{\beta \sigma_{b}\left(\sigma_{b^{\prime}}+\sigma_{b^{\prime \prime}}\right)} \rho_{0}\left(d \sigma_{b}\right)=\frac{e^{\beta\left(\sigma_{b^{\prime}}+\sigma_{b^{\prime \prime}}\right)}+e^{-\beta\left(\sigma_{b^{\prime}}+\sigma_{b^{\prime \prime}}\right)}}{2}
$$

in such a way that the contribution of the spins in $H_{R}$ does not appear in the integral anymore, because the set $\left\{\left(b^{\prime}, b^{\prime \prime}\right), b \in H_{R}\right\}$ is $L_{R}$. To get a more standard Ising representation, we would like to obtain now a coupling interaction between the spins in $L_{R}$. To do so, write

$$
\frac{e^{\beta\left(\sigma_{b^{\prime}}+\sigma_{b^{\prime \prime}}\right)}+e^{-\beta\left(\sigma_{b^{\prime}}+\sigma_{b^{\prime \prime}}\right)}}{2}=K e^{\beta^{\prime} \sigma_{b^{\prime}} \sigma_{b^{\prime \prime}}}
$$

where $K$ cancels by normalization. On the event $\left\{\sigma_{b^{\prime}}=+1, \sigma_{b^{\prime \prime}}=+1\right\}$, we should have

$$
\cosh [2 \beta]=K e^{\beta^{\prime}}
$$

and on the events $\left\{\sigma_{b^{\prime}}=-1, \sigma_{b^{\prime \prime}}=+1\right\}$ and $\left\{\sigma_{b^{\prime}}=+1, \sigma_{b^{\prime \prime}}=-1\right\}$,

$$
1=K e^{-\beta^{\prime}}
$$

Hence, one could take $K=e^{\beta^{\prime}}$ and $e^{2 \beta^{\prime}}=\cosh [2 \beta]$ i.e. $\beta^{\prime}=\frac{1}{2} \cosh ^{-1}\left(e^{2 \beta}\right)$ in (5.6), so that

$$
\left\langle\sigma_{1,1}\right\rangle^{+, \omega^{\prime} \tau_{R}}=
$$

$\frac{1}{\mathbf{Z}^{+, \omega^{\prime}, \tau_{R}}} \int_{\Omega_{L_{R}}} \sigma_{1,1} e^{\beta^{\prime} \sum_{\left\langle a a^{\prime}\right\rangle \subset L_{R}} \sigma_{a} \sigma_{a^{\prime}}+\beta^{\prime} \sum_{\left\langle a a^{\prime}\right\rangle, a \in L_{R}, a^{\prime} \in \lambda \backslash \Lambda_{R}} \sigma_{a} \tau_{a^{\prime}}} \rho_{L_{R}}\left[\left(d \sigma_{L_{R}}\right)\right.$. 


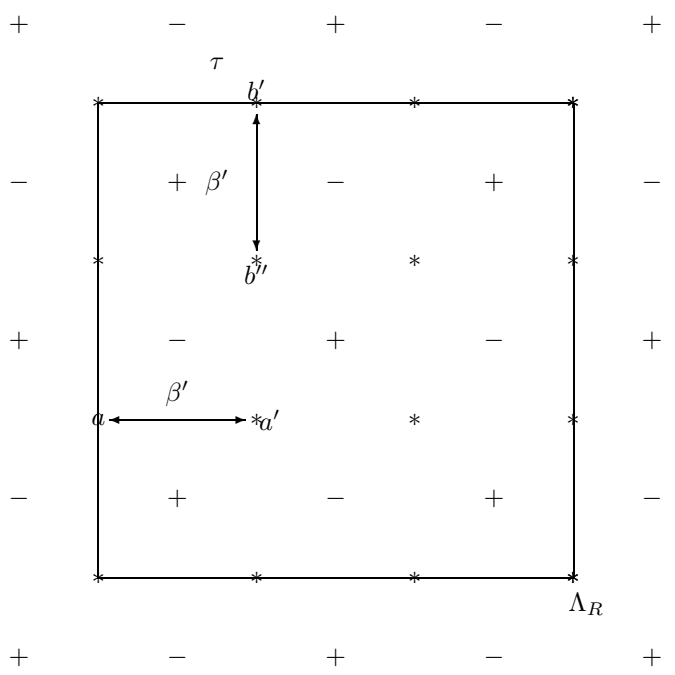

Figure 5 : Ising model on $2 \mathbb{Z}^{2}$ with coupling $\beta^{\prime}$.

This is exactly the magnetization of a ferromagnetic Ising model at inverse temperature $\beta^{\prime}$ on $2 \mathbb{Z}^{2}$, with the boundary condition $\tau$ on $\left(\lambda \backslash \Lambda_{R}\right) \cap$ $2 \mathbb{Z}^{2}$ and without external field. When the temperature is low enough, we know by Theorem 2.27 that a phase transition holds and that the above magnetization is an order parameter. This will eventually lead to essential discontinuity as soon as $\beta^{\prime}>\beta_{c}$, or equivalently $\beta>\tilde{\beta}_{c}=\frac{1}{2} \cosh ^{-1}\left(e^{2 \beta}\right)$.

To rigorously get the essential discontinuity, one should now do the same computation when $\Lambda_{n}$ is bigger than $\Lambda$, i.e for distinct neighborhoods of the alternating configuration, where all pluses or all minuses far away will create a external field that eventually selects the different phases. The procedure is the same but one has to be careful in some computations, to eventually give rise to the essential discontinuity we seek for. Thus, this failure of quasilocality comes directly from the presence of a phase transition in some "hidden system", that of the internal spins. This is carefully proved in detail in [36], through the following lemma

Lemma 5.7 (essential discontinuity). Let $\beta>\frac{1}{2} \cosh ^{-1}\left(e^{2 \beta_{c}}\right)$ and let $\omega^{\text {alt }}$ be the alternating configuration. $\forall \epsilon>0, \forall \mathcal{N}$ neighborhood of $\omega^{\text {alt }}, \exists R_{o}>0$ such that $\forall R>R_{o}$, we can find $\mathcal{N}_{R,+}, \mathcal{N}_{R,-} \subset \mathcal{N}$ with $\nu\left[A_{R,+}\right]=\nu\left[A_{R,-}\right]>0$ and for $\nu$-a.e. $\omega_{1}^{\prime} \in \mathcal{N}_{R,+}$, for $\nu$-a.e. $\omega_{2}^{\prime} \in \mathcal{N}_{R,-}$,

$$
\nu\left[\sigma_{0}^{\prime} \mid \mathcal{F}_{\{0\}^{c}}^{\prime}\right]\left(\omega_{1}^{\prime}\right)-\nu\left[\sigma_{0}^{\prime} \mid \mathcal{F}_{\{\mathbf{0}\}^{c}}^{\prime}\right]\left(\omega_{2}^{\prime}\right)>\epsilon .
$$


Thus, no version of the conditional probabilities of $\nu$ given $\mathcal{F}_{\{0\}^{c}}^{\prime}$ can be continuous.

This proves Theorem 5.1. This basic example expresses the link between the pathology and the existence of a phase transition in some "hidden" system. The same procedure has to be used for more general RG transformations, but it is sometimes difficult or even unknown to detect a bad configuration: Indeed, getting a bad configuration amounts to prove phase transitions, and this is sometimes, not to say often, difficult or unknown, involving e.g. sophisticated versions of the theory of Pirogov-Sinai. We give now a non-exhaustive catalogue of results that have been proved during the last decades, many other examples are rigorously described in $[36,33]$.

\subsubsection{General RG transformations, examples and re- sults}

In the general framework, we deal with two configuration spaces, the socalled original space $(\Omega, \mathcal{F}, \rho)$ and a so-called image space $\left(\Omega^{\prime}, \mathcal{F}^{\prime}, \rho^{\prime}\right)$. Most of the time, the lattice $S^{\prime}$ of the image system is smaller, and of the same kind (e.g. $S=\mathbb{Z}^{d}, S^{\prime}=\mathbb{Z}^{d^{\prime}}, d \geq d^{\prime}$ ); the above decimation transformation is e.g. sometimes described with $2 \mathbb{Z}^{d}$ as image lattice, and the projection on an hyperplane or "restriction to a layer" is studied in this context of the renormalization group whereas it does not satisfy all the properties of our following formal definition ${ }^{10}$, whose extension to spaces of measures is standard.

Definition 5.8 (R.G. kernels). A renormalization group transformation $(R G T)$ is a probability kernel $T$ from $(\Omega, \mathcal{F})$ to $\left(\Omega^{\prime}, \mathcal{F}^{\prime}\right)$ such that

1. $T$ carries $\mathcal{M}_{1, \text { inv }}^{+}(\Omega)$ onto $\mathcal{M}_{1, \text { inv }}^{+}(\Omega)$.

2. There exist sequences of cubes $\left(\Lambda_{n}\right)_{n \in \mathbb{N}}$ and $\left(\Lambda_{n}^{\prime}\right)_{n \in \mathbb{N}}$, respectively finite subsets of $S$ and $S^{\prime}$, such that:

(a) $\forall A^{\prime} \in \mathcal{F}_{\Lambda_{n}^{\prime}}^{\prime}$, the function $T\left(\cdot, A^{\prime}\right)$ is $\mathcal{F}_{\Lambda_{n}}$-measurable: The behavior of the image spins in $\Lambda_{n}^{\prime}$ depends only on the original spins in $\Lambda_{n}$.

(b) $\limsup _{n \rightarrow \infty} \frac{\left|\Lambda_{n}\right|}{\left|\Lambda_{n}^{\prime}\right|} \leq K<\infty$.

We give now a few examples where non-Gibbsianness has been proved to arise. As already claimed, it is more illustrative than exhaustive, a general method applying to many examples is available in [36] and in related papers

\footnotetext{
${ }^{10}$ More precisely, our definition refers to block renormalization transformations and exclude momentum transformations, which do not satisfy the second requirement.
} 
from our bibliography. We distinguish two types of examples, those where the transformation is deterministic, like the above decimation, and the more general stochastic ones. We first extend the previous results to more general decimations.

\section{Deterministic transformations}

A RGT is said to be deterministic when the probability kernel induced by $T$ is deterministic in the sense that

$$
\forall A^{\prime} \in \mathcal{F}^{\prime}, \forall \omega \in \Omega, T\left(\omega, A^{\prime}\right)=\delta_{\omega^{\prime}}\left(A^{\prime}\right)
$$

where the image $\omega^{\prime}=t(\omega)$ is a function of the original configuration $\omega$.

\section{Decimation transformations in higher dimensions:}

It is thus a deterministic probability kernel from $\Omega=E^{\mathbb{Z}^{d}}$ onto itself, with $t(\omega)=\omega^{\prime}$ and $\omega_{i}^{\prime}=\omega_{b i}$. In the same spirit of the phenomenon observed for the $2 \mathrm{~d}$ Ising model with the alternating configuration, but with much more difficult proofs in general, usually involving heavy machinery and tricks to find a special configuration and to prove it is a point of essential discontinuity. Among other results, one gets

Theorem 5.9. [36] Let $d \geq 2$ and $b \geq 2$. Then for all $\beta>\beta(d, b)$ sufficiently large, for any Gibbs measure $\mu$ for the standard n.n. homogeneous Ising model on $\mathbb{Z}^{d}$ with coupling $J>0$ and magnetic field $h=0$, the decimated measure $\nu=T_{b} \mu$ is not quasilocal.

This result is also extended in some open region $(\beta, h)$ of the phase diagram, e.g. to small magnetic field at dimension $d \geq 3$, for an adapted special configuration, see Section 4.3.6. in [36]. In view of the historical aim of renormalization group, which iterates the transformations to reach the presumably fixed critical point, it is interesting to mention that the quasilocality property could be recovered after iterating this decimation transformation [39], and that other positive results on conservation on Gibbisanness in other parts of the phase diagram exist [60].

\section{Deterministic majority-rule transformation:}

The configuration spaces are still identical and are those of the $d$ dimensional Ising model $\Omega^{\prime}=\Omega=\{-1,+1\}^{\mathbb{Z}^{d}}, d \geq 1$. Let $b \geq 1$ be an integer and let $B_{0} \in \mathcal{S}$ with $\left|B_{0}\right|$ odd. Define, $\forall i \in \mathbb{Z}^{d}, B_{i}$ to be $B_{0}$ translated by $b \cdot i$ : $B_{i}=B_{0}+b \cdot i$. We call this subsets of $\mathbb{Z}^{d}$ blocks. The deterministic kernel is the transformation $t(\omega)=\omega^{\prime}$ 
defined by

$$
\forall i \in \mathbb{Z}^{d}, \omega_{i}^{\prime}=\frac{\sum_{j \in B_{i}} \omega_{j}}{\left|\sum_{j \in B_{i}} \omega_{j}\right|}
$$

For these transformations, it is sometimes difficult, due to constraints on the blocks, to find bad configurations leading to non-quasilocality, see [36]. Nevertheless, one has the

Theorem 5.10. [36] Let $J$ large enough, $\mu$ any Gibbs measure for the $2 d$ Ising model with n.n. coupling $J$ and zero magnetic field. Let $T$ be the majority-rule with blocks of size $\left|B_{0}\right|=7$. Then $\nu=\mu T$ is not quasilocal.

The result has been extended for smaller blocks using a computerassisted proof in [68]. Such transformations belong to a more general family of block-spins transformations, very useful in renormalization procedures or multi-scale analysis, see e.g $[5,16]$.

\section{Majority-rule on a Cayley tree with overlapping blocks:}

The Ising model on a Cayley tree has been introduced in the previous chapter. We shall restrict ourselves to the simplest rooted-Cayley tree $\mathcal{T}_{0}^{2}[8]$ and let $\mu$ be any Gibbs measure for this model (we have seen in the previous chapter that there always exists at least one Gibbs measure for this model). We choose the root as the origin and we denote it $r$. Define $\Omega=\{-1,+1\}^{\mathcal{T}_{0}^{2}}$ and $\Omega^{\prime}=\{-1,0,+1\}^{\mathcal{T}_{0}^{2}}$, and let $R$ be any non negative integer to define the closed ball of $S$ of radius $R$ to be $V_{R}=\left\{i \in \mathcal{T}_{0}^{2} \mid d(r, i) \leq R\right\}$. Denote also its boundary by $W_{R}=\left\{i \in \mathcal{T}_{0}^{2} \mid d(r, i)=R\right\}$ where $d$ is the canonical metric on $\mathcal{T}_{0}^{2}$. We shall represent the vertices of $\mathcal{T}_{0}^{2}$ by sequences of bits, defined by recurrence: The representation of the origin $r$ is the void binary sequence, and that of its neighbors are chosen to be 0 and 1. Now let $R>0$ and let $i \in W_{R}$ with representation $i^{*}$. There are only two sites $k$ and $l$ in $W_{R+1}$ at distance 1 from $i$. We define then their representations to be $k^{*}=i^{*} 0$ and $l^{*}=i^{*} 1$, obtaining a representation of each vertex of $\mathcal{T}_{0}^{2}$. We shall now write the same symbol $i$ for the vertex or its binary representation $i^{*}$. Define $C_{r}=\{r, 0,1\}$ and $\forall j \in \mathcal{T}_{0}^{2}, j \neq r$, the cell

$$
C_{j}=\{j, j 0, j 1\}
$$

where $j 0$ and $j 1$ are the two neighbors of $j$ from the "following" level. For example, $C_{0}=\{0,00,01\}$. Define as well $c_{j}=\operatorname{Card}\left(C_{j}\right)$, with here $c_{j}=c=3$, and consider now the deterministic transformation 
$t: \omega \longmapsto t(\omega)=\omega^{\prime}$ where $\omega^{\prime}$ is defined by

$$
\omega_{j}^{\prime}=\left\{\begin{array}{lll}
+1 & \text { iff } & \frac{1}{c} \sum_{i \in C_{j}} \omega_{i}=+1 \\
0 & \text { iff } & \frac{1}{c}\left|\sum_{i \in C_{j}} \omega_{i}\right|<1 \\
-1 & \text { iff } & \frac{1}{c} \sum_{i \in C_{j}} \omega_{i}=-1
\end{array}\right.
$$

This could be seen as a static version of the voter model, where a child votes like its parents only when they agree and does not formulate an opinion otherwise. In this case, due to the overlapping of the blocks, the failure of quasilocality occurs at all temperatures, for the very particular null everywhere configuration. An interesting fact is that the set of bad configurations is topologically rather big but suspected to be of zero DLR-measure [82].

Theorem 5.11. [82] Let $\mu$ be any Gibbs measure for the Ising model on $\mathcal{T}_{0}^{2}$ and let $\nu$ be the image of $\mu$ by $T$. Then $\nu$ is non quasilocal at any temperature and cannot be a Gibbs measure.

The previous result could be generalized to non-rooted Cayley trees, and with other sizes of blocks, also as a stochastic transformation, modelling the fact that a child does not always vote like its parents, as follows: Let $\xi$ be a Bernoulli random variable with parameter $\epsilon \in[0,1]$. Define the deterministic map $t_{\epsilon}: \omega \longmapsto t(\omega)=\omega^{\prime}$ where $\omega^{\prime}$ is defined for all $j \in \mathcal{T}_{0}^{2}$ by

$$
\omega_{j}^{\prime}=\left\{\begin{array}{lll}
+1 & \text { iff } & \frac{1}{c} \sum_{i \in C_{j}} \omega_{i}=+1 \text { and } \xi=1 \\
-1 & \text { iff } & \frac{1}{c} \sum_{i \in C_{j}} \omega_{i}=-1 \text { and } \xi=1 \\
0 & \text { if } \quad \xi=0
\end{array}\right.
$$

Its action is described by a probabilistic kernel $T_{\epsilon}$ defined by:

$$
\forall A^{\prime} \in \mathcal{F}^{\prime}, \forall \omega \in \Omega, T_{\epsilon}\left(\omega, A^{\prime}\right)=(1-\xi) \delta_{t_{\epsilon}(\omega)}\left(A^{\prime}\right)+\xi \delta_{0}\left(A^{\prime}\right) .
$$

An interesting study would be to analyze the effect of this extra randomness to the degree of non-Gibbsianness of the image measure.

\section{Restriction of Ising model to a layer [98, 112]:}

This transformation is not properly speaking a RGT in the sense of our definition, because of the lack of strict locality. Nevertheless, it is known to lead to non-Gibbsianness and is a good example of a new kind of random fields, the weakly Gibbsian measures, which will be introduced soon [98]. The configuration spaces are $\Omega=\{-1,+1\}^{\mathbb{Z}^{2}}$ and $\Omega^{\prime}=\{-1,+1\}^{\mathbb{Z}}$. The transformation is deterministic and defined by $t(\omega)=\omega^{\prime}$ where $\omega^{\prime}$ is defined by $\forall i \in \mathbb{Z}, \omega_{i}^{\prime}=\omega_{(i, 0)}$ where 0 
denotes here the origin in $\mathbb{Z}$. The interesting fact in this example, together with the fact that it could seem very natural at a first sight to consider the projected measure to be Gibbs, is that the original proof relies on wrong large deviation properties of the projected measure [112].

\section{Stochastic transformations}

In contrast to the deterministic case, a stochastic transformation could lead to different image configurations, with a certain probability for each. We have already seen above an example of stochastic RGT on the tree.

\section{Stochastic majority-rule for Ising model:}

The definition is very similar to the deterministic one, except that we deal with blocks $B_{0}$ with $\left|B_{0}\right|$ even. The configuration spaces are still $\Omega^{\prime}=\Omega=\{-1,+1\}^{\mathbb{Z}^{d}}, d \geq 1$. Let $b \geq 1$ be an integer and let $B_{0} \in \mathcal{S}$ with $\left|B_{0}\right|$ even. Define, $\forall i \in \mathbb{Z}^{d}, B_{i}$ to be translate of $B_{0}$ by $b \cdot i$ : $B_{i}=B_{0}+b \cdot i$. Let $\xi$ be a Bernoulli random variable on $(\Omega, \mathcal{F})$ with parameter $p$, most of the time considered to be $p=\frac{1}{2}$. The stochastic majority-rule is the transformation $T$ which transforms $\omega$ in $t(\omega)=\omega^{\prime}$ with

$$
\omega_{i}^{\prime}=\left\{\begin{array}{ccl}
+1 & \text { if } \quad \sum_{j \in B_{i}} \omega_{j}>0 \\
-1 & \text { if } \quad \sum_{j \in B_{i}} \omega_{j}<0 \\
+1 & \text { if } \quad \sum_{j \in B_{i}} \omega_{j}=0 \text { and } \xi=+1 \\
-1 & \text { if } \quad \sum_{j \in B_{i}} \omega_{j}=0 \text { and } \xi=0
\end{array}\right.
$$

\section{Kadanoff transformations for the Ising model:}

These transformations model a lot of interesting and historical RGT. We shall not deal with them, but some are widely studied in $[56,63$, 36]. Here again the blocks $B_{i}$ are defined in the same way for $i \in \mathbb{Z}^{d}$, the configuration spaces are $\Omega=\Omega^{\prime}=\{-1,+1\}^{\mathbb{Z}^{d}}$ and $p$ is a strictly positive real. The RGT map is defined atom per atom by

$$
T\left(\omega, \omega^{\prime}\right)=\prod_{i \in \mathbb{Z}^{d}} \frac{\exp \left(p \omega_{i}^{\prime} \sum_{j \in B_{i}} \omega_{j}\right)}{2 \cosh \left(p \sum_{j \in B_{i}} \sigma_{j}\right)}
$$

This transformation is also associated with stochastic evolutions of Gibbs measures, as we shall see. They have been proved to lead to non-Gibbsianness for $d \geq 2, b \geq 1$ and $p$ finite. It also includes majority rules or decimations in the limit $p \rightarrow \infty$ for suitable blocks.

Many other examples are available in the literature, and as claimed in [33], the surprise is eventually not that they are non-Gibbsian, but that 
it took so long to realize it, the set of Gibbs measures being topologically very small [65]. In the same seminal paper [36], positive general results are given about the action of these transformations on Hamiltonians and potentials, excluding various scenarii related to figure 1. Here we only quote them. See the discussions in [43] and [36].

\section{Renormalization transformation on potentials}

As explained in the beginning of this chapter, the extension of the renormalization transformations to potentials is not always well-defined, whereas the extension of the RGT to an action on measures is standard and always possible. Nevertheless, two positive results have been proved by van Enter et al., and we introduce them before describing the pathologies of the renormalization group. We restrict ourselves to a space $\mathcal{B}^{1}$ consisting of the translation-invariant, continuous and uniformly absolutely convergent potentials. We introduce first a relation instead of a function:

Definition 5.12 (RGT on interactions). Let $T$ be an $R G T$. We define a $R G$-relation $\mathcal{R}=\mathcal{R}_{T}$ on interactions by the relation

$$
\mathcal{R}=\left\{\left(\Phi, \Phi^{\prime}\right) \in \mathcal{B}^{1} \times \mathcal{B}^{1}: \exists \mu \in \mathcal{G}_{\text {inv }}\left(\gamma^{\Phi}\right) \text { s.t. } \mu T \in \mathcal{G}\left(\gamma^{\Phi^{\prime}}\right)\right\}
$$

where $\mu T$ is the image measure of $\mu$ by $T$.

The next theorem tells us that $\mathcal{R}$ is single-valued and is proved in [36].

Theorem 5.13 (first fundamental theorem of the renormalization group). Let $\mu$ and $\nu$ be translation-invariant Gibbs measures with respect to the same interaction $\Phi \in \mathcal{B}^{1}$ and let $T$ be a $R G T$. The following results are true:

1. Either $\mu T$ and $\nu T$ are both non-quasilocal, or else there exists a quasilocal specification $\gamma^{\prime}$ with which both $\mu T$ and $\nu T$ are consistent.

2. Either $\mu T$ and $\nu T$ are both non-Gibbsian, or else there exists a uniformly absolutely convergent potential $\Phi^{\prime}$ for which both $\mu T$ and $\nu T$ are Gibbs measures.

\subsubsection{Stochastic evolutions of Gibbs measures}

Once these RG pathologies have been identified as the manifestation of non-Gibbsianness, it seemed natural to investigate this question for stochastic Ising models, introduced in the previous chapter. For example, due to the equilibrium considerations that led to the introduction of the Gibbs property, it should be plausible to encounter a failure of Gibbsianness in the course of stochastic evolutions of Gibbs measures, in particular when the process is out of equilibrium. It is indeed the case during the heating 
of a low temperature Ising model, i.e. the stochastic evolution of a low temperature Gibbs measure for the ferromagnetic n.n. Ising model during a high temperature Glauber dynamics. It is not so simple to establish, and not always true; physical interpretations can be found in [105]. Nevertheless, not much is known in non-equilibrium statistical mechanics, so any information about Gibbsianness in transient regimes is welcome. The first systematical study of such phenomena has been made in [35], although similar investigations had been made earlier in [99, 89]. Before describing a bit more the relationships with (stochastic) RG-transformations through the description of an infinite-temperature Glauber dynamics, let us quote their general result.

Theorem 5.14. [35] Let $\Phi$ be a translation-invariant potential, $\mu$ a corresponding translation-invariant Gibbs measure at inverse temperature $\beta$ and $(S(t))_{t>0}$ the semi-group corresponding to a dynamics having $\mu$ as reversible measure. Denote by $\nu$ an initial translation-invariant Gibbs distribution of the a priori configuration. Then

1. For all $\nu, \mu$, the time-evolved measure $\nu S(t)$ is Gibbs for small times $t \leq t_{0}(\beta)$.

2. If $\mu, \nu$ corresponds to high or infinite temperature Gibbs measures, then the time evolved measure is Gibbs for all times $t$.

3. If $\nu$ is a low temperature Gibbs measure for some t.i. potential whereas $\mu$ is a high temperature Gibbs measure, then the time evolved measure is Gibbs for large $t$. When $\nu$ is not a zero temperature Gibbs measure and $\mu$ corresponds to a high temperature with a small magnetic filed, Gibbsianness is recovered for larger times.

For the sake of simplicity, we describe the results for infinite temperature Glauber dynamics of low temperature phases of the Ising model at dimension $d \geq 2$. Starting from the + -phase $\mu_{\beta}^{+}$of the Ising model at low enough temperature $\beta^{-1}>0$, we apply a stochastic spin-flip dynamics at rate 1 , independently over the sites. The time evolved measure is then formally ${ }^{11}$

$$
\mu_{\beta, t}(\eta):=\sum_{\sigma \in \Omega} \mu_{\beta}^{+}(\sigma) \prod_{i \in \mathbb{Z}^{d}} \frac{e^{\eta_{i} \sigma_{i} h_{t}}}{2 \cosh h_{t}}, \text { with } \mathrm{h}_{\mathrm{t}}=\frac{1}{2} \log \frac{1+\mathrm{e}^{-2 \mathrm{t}}}{1-\mathrm{e}^{-2 \mathrm{t}}} .
$$

The product kernel in (5.15) is a special case of a Glauber dynamics for infinite temperature $[35,84]$, its particular form in terms of a dynamical magnetic field $h_{t}$ being obtained by a tricky use of the small size of

\footnotetext{
${ }^{11}$ The summation does not make sense like it is here, because it is perfomed over the uncountable configuration space $\Omega$. To rigorously define it, one can e.g. work at finite volume first and consider the thermodynamic limit.
} 
$E=\{-1,+1\}$. This last particular form allows to interpret these dynamics as a Kadanoff-like transformation. It is known that the time-evolved measure $\mu_{\beta, t}$ tends to a spin-flip invariant product measure on $\{-1,+1\}^{\mathbb{Z}^{d}}$, with $t \uparrow \infty$, which is trivially non-null and quasilocal, and thus Gibbs. Nevertheless, the Gibbs property is lost during this evolution and recovered only at equilibrium:

Theorem 5.16. [35] Assume that the initial temperature $\beta^{-1}$ is smaller than the critical temperature of the n.n. Ising model for $d \geq 2$. Then there exists $t_{0}(\beta) \leq t_{1}(\beta)$ such that:

1. $\mu_{\beta, t}$ is a Gibbs measure for all $0 \leq t<t_{0}(\beta)$.

2. $\mu_{\beta, t}$ is not a Gibbs measure for all $0<t_{1}(\beta) \leq t<+\infty$.

Non-Gibbsianness is here related to the possibility of a phase transition in some constrained model ${ }^{12}$. There remains a large interval of time where the validity of the Gibbs property of the time-evolved measure remains unknown for this lattice model [61]. This has motivated the study of similar phenomena for mean-field models in [77], where the sharpness of the Gibbs/non-Gibbs transition has been proved. This study has required the introduction of the new notion of Gibbsianness for mean-field models, see e.g. $[59,75]$, and the relationships between lattice and mean-field results encourages us to investigate it further on. Moreover, Gibbianness for short times has been established for more general local stochastic evolutions in [84] and the large deviation properties have even been proved to be conserved during the evolution for the special case of the Glauber dynamics in [85]. Other sources of non-Gibbsianness during stochastic evolutions, but concerning the stationary and not the transient regime, have been investigated in $[89,23,47]$ for e.g. discrete dynamics or probabilistic cellular automata. Similar considerations have led to a short review about the relationships between non-Gibbsianness and disordered systems in [37].

\subsubsection{Joint measure of short range disordered systems}

This example has allowed substantial progress in the Dobrushin program of restoration of Gibbsianness, and reinforces our philosophy of focusing on continuity properties of conditional probabilities rather than on convergence properties of a potential, as we shall see in next Section. NonGibbsianness has here also been very useful to explain pathologies in the so-called Morita approach to disordered systems, see [79, 76] in the proceedings volume [38]. The Random Field Ising Model (RFIM) is an Ising model

\footnotetext{
${ }^{12}$ The constrained model is a three dimensional Random Field Ising Model, due to the randomness of the dynamical field. The possible occurrence of phase transitions for this model has been proved in [13].
} 
where the magnetic field $h$ is replaced by (say i.i.d. \pm 1 ) random variable $\eta_{i}$ of common law $\mathbb{P}$ at each site of the lattice. For a given $\eta=\left(\eta_{i}\right)_{i \in S}$, whose law is also denoted by $\mathbb{P}$, the corresponding ("quenched") Gibbs measures depend on this disorder and are denoted by $\mu[\eta]$. The Morita approach [79] considers the joint measure "configuration-disorder", formally defined by $K(d \eta, d \sigma)=\mu[\eta](d \sigma) \mathbb{P}(d \eta)$, to be a Gibbs measure for a potential of the joint variables, but it has been proved in [73] that this measure can be non-Gibbs for $d \geq 2$ and for a small disorder. The mechanism, although more complicated, is similar to the previous examples, and the arising of non-quasilocality is made possible when a ferromagnetic ordering is itself possible in the quenched system, and thus the conditions on $d$ and on the disorder are those required for such a phase transition to hold in [13]. A diluted and simpler version of this phenomenon concerns the GriSing random field, whose link with Griffiths's singularities is also very relevant for its similarities with RG pathologies [40].

\subsubsection{Other sources of non-Gibbsianness}

Soon after the detection of the renormalization group pathologies as the manifestation of the occurrence of non-Gibbsianness, the latter phenomenon has been detected in many other areas of probability theory and statistical mechanics, like Hidden Markov models, Random-cluster model, convex combinations of product measures, etc. see [36] or references in $[38]$.

Before using these examples to emphasize how important are the continuity properties of conditional probabilities in the Gibbs formalism, we describe some recent extensions of the Gibbs property within the so-called Dobrushin program of restoration of Gibbsianness.

\subsection{Generalized Gibbs measures}

In 1995, in view of the RG pathologies described in [36] and in the physics literature, Dobrushin launched a program of restoration of Gibbsianness consisting in two parts [30]:

1. To give an alternative (weaker) definition of Gibbsianness that would be stable under scaling transformations.

2. To restore the thermodynamic properties of these measures in order to get a proper definition of equilibrium states. 


\subsubsection{Dobrushin program of restoration, Part I}

The first part of this program mainly yields two different restoration notions, depending if one focus on a relaxation of the convergence properties of the potential, that leads to weak Gibbsianness, or on a relaxation on the topological properties of conditional probabilities, leading then to almost Gibbsianness or almost quasilocality. The first, reminiscent to the notion chosen to describe systems with hard-core exclusions or with unbounded spins [86], appeared to be indeed weaker than the latter. It express consistency w.r.t. an almost surely convergent potential:

Definition 5.17 (Weakly Gibbs). A probability measure $\mu \in \mathcal{M}_{1}(\Omega)$ is said to be weakly Gibbs if there exists a potential $\Phi$ and a tail-measurable set $\Omega_{\Phi}$ on which $\Phi$ is convergent, of full measure $\mu\left(\Omega_{\Phi}\right)=1$, and such that $\mu \in \mathcal{G}\left(\gamma^{\Phi}\right)$.

Tail-measurability is required to ensure that the partition function is well-defined. Weak Gibbsianness has been proved for most of the renormalized measures of the previous section [14, 95, 97, 98] and relies on the existence of the already mentioned relative energies [99], that usually enables to prove the almost sure convergence of a telescoping potential of Kozlov type. A non-Gibbsian measure arising in stochastic evolutions has also been proved to be weakly Gibbsian [23] and joint measures of disordered systems too [74]. It actually seems to be not common in our context to find a transformation of a Gibbs measure that is not weakly Gibbs, although examples such as convex combinations of product measures exist $[97,96]$. Moreover, in such a case, the almost sure convergence of the potential does not tell much about the crucial continuity properties of conditional probabilities. This has motivated the second main restoration notion:

Definition 5.18 (Almost Gibbs). A probability measures $\mu$ is almost Gibbs if its finite-volume conditional probabilities are continuous functions of the boundary conditions, except on a set of $\mu$-measure zero, i.e. if there exists a specification $\gamma$ such that $\mu \in \mathcal{G}(\gamma)$ and $\mu\left(\Omega_{\gamma}\right)=1$.

An almost-sure version of Kozlov-Sullivan's use of the inclusion-exclusion principle in the Gibbs representation theorem proves that almost Gibbs implies weak Gibbs $[72,117,97]$, but the converse is not true (see e.g. [91]). The decimated measure has been proved to be almost Gibbsian in [45], and the method applies to other renormalized measures [46], but not for the projection to a layer for which the problem is open. More interestingly, the contrary (almost-sure non-quasilocality) has been proved [74] for the joint measure of the RFIM, which even has a set of bad configurations with full measure. The peculiarity of this example appeared to be a good warning for the importance of quasilocality in the characterization of equilibrium states for lattice spin systems. In particular, it discriminates the 
weak Gibbs restoration from an almost Gibbs one, due to the consequences it has on the thermodynamic properties of the corresponding measures, in the second part of the Dobrushin program.

Other restoration notions (robust Gibbsianness [39], fractal quasilocality [82], Asymptotically sub-volumic measures [100]) were introduced in the course of the preceding studies. More recently, an intermediate between the almost Gibbs and weak Gibbs has been introduced, and seems to be a very relevant starting definition of generalized Gibbs measure, called Intuitively weak Gibbsianness in [41]:

Definition 5.19 (Intuitively weak Gibbs). A weakly Gibbsian measure $\mu \in \mathcal{M}_{1}^{+}(\Omega)$ is said to be intuitively weak Gibbs if there exists a set $\Omega_{1} \subset$ $\Omega_{\Phi}$ with $\mu\left(\Omega_{1}\right)=1$ and s.t. for all $\sigma \in \Omega$,

$$
\gamma_{\Lambda}^{\Phi}\left(\sigma_{\Lambda} \mid \omega_{\Lambda_{n} \backslash \Lambda} \eta_{\Lambda^{c}}\right) \underset{\Lambda \uparrow \mathcal{S}}{\longrightarrow} \gamma_{\Lambda}^{\Phi}(\sigma \mid \omega)
$$

for all $\omega, \eta \in \Omega_{1}$.

The notion is intermediate between weak and almost Gibbsianness, the difference between all these notions being that the convergence (5.20) holds:

- For all $\omega$ and all $\eta$ when $\mu$ is Gibbs (quasilocal).

- For $\mu$-a.e $\omega$ and all $\eta$ when $\mu$ is almost Gibbs.

- For $\mu$-a.e $\omega$ and $\mu$-a.e $\eta$ when $\mu$ is intuitively weak Gibbs.

The thermodynamic properties of Gibbs measures have been partially restored for almost Gibbsian measures, whereas weak Gibbsianness seems to be indeed too weak to be a satisfactory notion from this point of view.

\subsubsection{Dobrushin program of restoration, part II}

This second part aims thus at the restoration of the thermodynamic properties of Gibbs measures, mostly in terms of a variational principle involving thermodynamic functions like entropy, pressure,etc. In our framework in particular, one would like to identify the equilibrium states as minimizers of the free energy of the system, in virtue of the second law of thermodynamics. To do so, one should first recover well-defined thermodynamic functions at infinite volume for the new families of random fields introduced above. In the weakly Gibbsian context, this can be done directly but their existence has to be restricted to typical boundary conditions, see $[90,98,99]$, whereas in the case of renormalized measures, the useful notion of asymptotically decoupled measures introduced by Pfister [104] ensures their existence and the validity of a large deviation principle: 
Definition 5.21. A measure $\mu \in \mathcal{M}_{1 \text {,inv }}^{+}(\Omega)$ is asymptotically decoupled if there exists functions $g: \mathbb{N} \longrightarrow \mathbb{N}$ and $c: \mathbb{N} \longrightarrow[0, \infty)$ s.t.

$$
\lim _{n \rightarrow \infty} \frac{g(n)}{n}=0 \text { and } \lim _{n \rightarrow \infty} \frac{c(n)}{\left|\Lambda_{n}\right|}=0
$$

and for all $n \in \mathbb{N}, A \in \mathcal{F}_{\Lambda_{n}}, B \in \mathcal{F}_{\Lambda_{n+g(n)}^{c}}$

$$
e^{-c(n)} \mu(A) \mu(B) \leq \mu(A \cap B) \leq e^{c(n)} \mu(A) \mu(B) .
$$

This class strictly contains the set of all Gibbs measures and this property is conserved under the local RGT considered in these lectures, ensuring then the existence of the thermodynamic functions considered by Pfister ([104]) for the renormalized measures. Nevertheless, we emphasize that the existence of relative entropy is still an open problem for the projection of the Ising model to a layer [45, 46, 98].

Thus, our renormalized measures satisfy the thermodynamical variational principle whereas the specification independent one has been fully restored for the decimated measure in [45], using ideas taken from the concept of global specification [44]. As a corollary this has established the almost Gibbsianness of this measure, as we see now. Keeping the same notation and writing $\nu^{+}$and $\nu^{-}$the decimation of the + and - phases of the $2 \mathrm{~d}$ Ising model, one has:

Theorem 5.22. [45] Consider the decimation of the Ising model at $\beta>$ $\beta_{c}$. Then

1. For every $\mu \in \mathcal{M}_{1, \text { inv }}^{+}(\Omega), h\left(\mu \mid \nu^{+}\right)$exists and $h\left(\nu^{-} \mid \nu^{+}\right)=0$.

2. $\nu^{-} \in \mathcal{G}_{\text {inv }}\left(\gamma^{+}\right)$, where $\gamma^{+}$specifies $\nu^{+}$, and they are almost Gibbs.

3. If $h\left(\mu \mid \nu^{+}\right)=0$ and $\mu\left(\Omega_{\gamma^{+}}\right)=1$ then $\mu \in \mathcal{M}_{1, \text { inv }}^{+}(\Omega)$.

The proof relies on the general criterion given in Chapter 4, also used to partially restore the second part of the variational principle in [78].

Another relevant feature described in [78] is the important role played by the joint measure of the RFIM to discriminate the restoration notion expressed in terms of potentials (weak Gibbsianness) from topological ones (e.g. almost quasilocality). Indeed, this joint measure, already identified to be one of the physically relevant known examples of weakly Gibbsian measure that is not almost quasilocal, also provides an example where the (specification-dependent) variational principle fails ${ }^{13}$. In this situation,

\footnotetext{
${ }^{13}$ Depending on the boundary condition used to perform the thermodynamic limit, one gets two measures described by a different system of conditional probabilities but with zero relative entropy.
} 
we have two candidates to represent equilibrium states, corresponding to different interactions each one saturating the variational principle of the other, which can be easily seen to be physically meaningless. The fact that this happens for a weakly and non almost Gibbsian measure clearly indicates that one has to insist on continuity properties of the conditional probabilities in order to restore the Gibbs property in the framework of the Dobrushin program. Together with the Gibbs representation theorem, this also emphasizes the relevance of the description of Gibbs measures in the quasilocal framework, i.e. in terms of topological properties of conditional probabilities rather than in terms of potentials. These observations have motivated a new "DLR-like" approach to mean-field models, initiated recently in $[59,75,77,83]$.

\section{Acknowledgements:}

The proof of the variational principle for quasilocal measures has been corrected from [78] in collaboration with C. Külske (Groningen) and F. Redig (Leiden). We thank the anonymous referee of the present paper who pointed out a possible problem and gave hints to solve it. The author also thanks the members of the departments of mathematics of the universities UFMG and UFGRS who organized and attended to these lectures, A.C.D. van Enter (Groningen) for its legendary careful readings and suggestions, S. Friedli (Belo Horizonte) for many comments, readings and discussions and Feijia Wang (Leiden) for questions and suggestions. 


\section{Bibliography}

[1] M. Aizenman. Translation-invariance and instability of phase coexistence in the two-dimensional Ising system. Comm. Math. Phys. 73, no 1:83-94, 1980.

[2] H. van Beijeren. Interface sharpness in the Ising model. Comm. Math. Phys. 401-6, 1975.

[3] P. Berti, P. Rigo. 0-1 laws for regular conditional distributions. Ann. Prob. 35:649-662, 2007.

[4] P. Berti, P. Rigo. A conditional 0-1 law for the symmetric $\sigma$ field. J. Theo. Proba. 21:517-526, 2008.

[5] L. Bertini, E. Cirillo, E. Olivieri. Renormalization group in the uniqueness region: weak Gibbsianity and convergence. Comm. Math. Phys. 261, no. 2:323-378, 2006.

[6] P. Billingsley. Ergodic theory and information. John Wiley, New York-London-Sydney, 1965.

[7] P. Billingsley. Probability and measures. 3rd ed. Wiley series in probability and mathematical statistics. New York: Wiley, 1968 and 1995.

[8] P.M. Bleher, N.N. Ganihodgaev. On pure phases of the Ising model on the Bethe lattice. Theo. Prob. Appl. 35:1-26, 1990.

[9] T. Bodineau. Translation-invariant Gibbs states for the Ising model. Prob. Th. Relat. Field. 135, no. 2, 153-168, 2006.

[10] L. Boltzmann. Leçons sur la théorie des gaz, Gauthier-Villars, Paris, tome I 1902, tome II 1905. Réédition Jean Gabay, Paris, 1987.

[11] L. Boltzmann. The second law of Thermodynamics. In Ludwig Boltzmann, Theoretical Physics and Philosophical Problems, B. Mc Guinness eds, Reidel, 1974. 
[12] A. Bovier, M. Zahradník. The low temperature phase of KacIsing models. J. Stat.Phys. 87, nos 1/2:311-332, 1997.

[13] J. Bricmont, A. Kupiainen. Phase transition in the 3d Random Field Ising Model. Comm. Math. Phys. 142:539-572, 1988.

[14] J. Bricmont, A. Kupiainen, R. Lefevere. Renormalization group pathologies and the definition of Gibbs states. Comm. Math. Phys. 194, no. 2:359-388, 1998.

[15] R. Burton, C.-E. Pfister, J. Steif. The Variational Principle for Gibbs States Fails on Trees. Mark. Proc. Relat. Fields 1, no. 3:387-406, 1995.

[16] F. Camia, C.M. Newman, V. Sidoravicius. A particular bit of universality: scaling limits of some dependent percolation models. Comm. Math. Phys. 246, no. 2:311-332, 2004.

[17] J. Cardy. Scaling and renormalization in statistical physics. Cambridge Lecture Notes in Physics 5. Cambridge University Press, Cambridge, 1996.

[18] M. Cassandro, E. Presutti. Phase transitions in Ising systems with long but finite range interactions. Mark. Proc. Relat. Fields 2, no 2:241-262, 1996.

[19] F. Comets. Large Deviation Estimates for a Conditional Probability Distribution. Applications to Random Interaction Gibbs Measures. Prob. Th. Relat. Fields. 80:407-432, 1986.

[20] I. Csiszár. Information-type measures of difference of probability distributions and indirect observations. Studia Sci. Math. Hungar. 2:299-318, 1967.

[21] K.L. Chung. Markov chains with stationary transition probabilities. Second edition, Springer-Verlag, New-York, 1987.

[22] A. De Masi. Systems with long range interactions. Progress in Probability 54:25-81, Birkhauser, 2003.

[23] J. Depoorter, C. Maes. Stavskaya's measure is weakly Gibbsian. Mark. Proc. Relat. Fields 12, no 4: 791-804, 2006.

[24] C. Domb, M.S. Green (Eds). Phase transitions and Critical Phenomena, Vol. 6, Academic Press, NY, 1976.

[25] P. Diaconis. Recent progress on de Finetti's notions of exchangeability. Bayesian statistics 13:111-125, Oxford University Press, 1988. 
[26] R.L. Dobrushin. Existence of a phase transition in the twodimensional and three-dimensional Ising models. Dokl. Akad. Nauk SSSR 160:1046-1048 (Russian); translated as Soviet Physics Dokl.10:111-113, 1965.

[27] R.L. Dobrushin. Description of a random field by means of conditional probabilities and the conditions governing its regularity. Theor. Prob. Appl. 13:197-224, 1968.

[28] R.L. Dobrushin. Gibbs states describing coexistence of phases for a three-dimensional Ising model. Theo. Prob. Appl. 17, no 4:582-600, 1972.

[29] R.L. Dobrushin, E.A. Pecherski. A criterion for the uniqueness of Gibbsian fields in the non-compact case. In Probability theory and Mathematical Statistics, Lecture Notes in Mathematics 1021:97-110, Springer-Verlag, Berlin, 1983.

[30] R.L. Dobrushin, S.B. Shlosman. Gibbsian description of "non Gibbsian" field, Russian Math Surveys 52, 285-297, 1997. Also "Non-Gibbsian" states and their description. Comm. Math. Phys.200,no 1: 125-179, 1999.

[31] E.B. Dynkin. Sufficient statistics and extreme points. Ann. Proba. 6, No. 5:705-730, 1978.

[32] F.J. Dyson. An Ising ferromagnet with discontinuous long-range order. Comm. Math. Phys. 21:269-283, 1971.

[33] A.C.D. van Enter. On the possible failure of the Gibbs property for measures on lattice systems. Disordered systems and statistical physics: rigorous results (Budapest, 1995). Mark. Proc. Relat. Field. 2, no. 1:209-224, 1996.

[34] A.C.D. van Enter, R. Fernández. A remark on different norms and analycity for many particle interactions. J. Stat. Phys. 56: 965-972, 1989.

[35] A.C.D. van Enter, R. Fernández, F. den Hollander, F. Redig. Possible loss and recovery of Gibbsianness during the stochastic evolution of Gibbs measures. Comm. Math. Phys. 226, no. 1, 101-130, 2002

[36] A.C.D. van Enter, R. Fernández, A.D. Sokal. Regularity properties and pathologies of position-space renormalization-group transformations: Scope and limitations of Gibbsian theory. $J$. Stat. Phys. 72:879-1167, 1993. 
[37] A.C.D. van Enter, C. Külske. Two connections between random systems and non-Gibbsian measures. J. Stat. Phys. 126, no. 45:1007-1024, 2007.

[38] A.C.D. van Enter, A. Le Ny, F. Redig (eds). Proceedings of the workshop "Gibbs vs. non-Gibbs in statistical mechanics and related fields" (Eurandom 2003). Mark. Proc. Relat. Fields 10, no 3, 2004.

[39] A.C.D. van Enter, J. Lörinczi. Robustness of the non-Gibbsian property: some examples. J. Phys. A 29, no. 10:2465-2473, 1996.

[40] A.C.D. van Enter, C. Maes, R.H. Schonmann, S.B. Shlosman. The Griffiths singularity random field. in On Dobrushin's way. From probability theory to statistical physics, Amer. Math. Soc. Transl. Ser. 2, 198: 51-58, Amer. Math. Soc., Providence, RI, 2000

[41] A.C.D. van Enter, E.A. Verbitskiy. On the variational principle for generalized Gibbs measures. Mark. Proc. Relat. Fields 10, no 3:411-434, 2004.

[42] W. Feller. An introduction to probability theory and its applications, volume I. Wiley publications in statistics, 1957.

[43] R. Fernández. Gibbsianness and non-Gibbsianness in Lattice random fields. In Mathematical Statistical Physics. Proceedings of the 83rd Les Houches Summer School (july 2005), Elsevier, A. Bovier, A.C.D. van Enter, F. den Hollander, F. dunlop eds., 2006 .

[44] R. Fernández, C.-E. Pfister. Global specifications and nonquasilocality of projections of Gibbs measures. Ann. Proba. 25, no 3:1284-315, 1997.

[45] R. Fernández, A. Le Ny, F. Redig. Variational principle and almost quasilocality for renormalized measures. J. Stat. Phys. 111, nos 1/2:465-478, 2003.

[46] R. Fernández, A. Le Ny, F. Redig. Restoration of Gibbsianness for projected and FKG renormalized measures. Bull. Braz. Math. Soc. 34:437-55, 2003.

[47] R. Fernández, A. Toom. Non-Gibbsianness of the invariant measures of non-reversible cellular automata with totally asymmetric noise. Geometric methods in dynamics. II. Astrisque No. 287,2003 
[48] M.E. Fisher. Scaling, universality and renormalization group theory, in Critical phenomena (Stellenbosch 1982), Lecture Notes in Physics no 186:1-139, F.J.Hahne ed., Springer-Verlag, Berlin, 1983.

[49] J. Fröhlich, T. Spencer. The phase transition in the onedimensional Ising model with $1 / r^{2}$ interaction energy. Comm. Math. Phys. 84, no. 1:87-101, 1982 .

[50] K. Gawedski. Rigorous renormalization group at work. Physica 140A: 78-84, 1986.

[51] J.W. Gibbs. Elementary principles in statistical mechanics. Yale University Press, New Haven, 1902.

[52] H.O. Georgii. Gibbs Measures and Phase Transitions. Walter de Gruyter (de Gruyter Studies in Mathematics, Vol. 9), BerlinNew York, 1988.

[53] H.O. Georgii, Y. Higuchi. Percolation and number of phases in the two-dimensional Ising model. Probabilistic techniques in equilibrium and non-equilibrium statistical physics. J. Math. Phys. 41, no 3:1153-1169, 2000.

[54] W.T. Grandy. Entropy and the Time Evolution of Macroscopic Systems. International Series of Monograph on Physics 141, Oxford Science publications, 2008.

[55] R.B. Griffiths. Peierls proof of spontaneous magnetization in a two-dimensional Ising ferromagnet. Phys. Rev. 2, 136:A437A439, 1964.

[56] R.B. Griffiths, P.A. Pearce. Mathematical properties of position-space renormalization-group transformations, J. Stat. Phys. 20: 499-545, 1979.

[57] Y. Higuchi. Remarks on the limiting Gibbs states on a $(d+1)$ tree. Publ. RIMS, Kyoto Univ. 13:335-348, 1977.

[58] Y. Higuchi. On the absence of non-translation-invariant Gibbs states for the two-dimensional Ising model. Random fields, Vol I, II (Esztergom, 1979), Colloq. Math. Soc. Janos Bolyai 27:517-534, 1981.

[59] O. Häggström, C. Külske. Gibbs property of the fuzzy Potts model on trees and in mean-field. Mark. Proc. Relat. Fields 10 no 3:477-506, 2004. 
[60] K. Haller, T. Kennedy. Absence of renormalization group pathologies near the critical temperature. Two examples. J. Stat. Phys. 85, no. 5-6:607-637, 1996.

[61] F. den Hollander. Gibbs under stochastic dynamics ? Mark. Proc. Relat. Fields 10, no 3:507-516, 2004.

[62] D. Ioffe. Extremality of the disordered state for the Ising model on general trees. Progress in probability 40:3-14, B. Chauvin, S. Cohen, A. Rouault eds, 1996.

[63] R.B. Israel. Banach algebras and Kadanoff transformations, in Random Fields (Esztergom, 1979) J. Fritz, J.L. Lebowitz, and D.Szász eds, Vol II, pp.593-608, 1981.

[64] R.B. Israel. Convexity in the theory of lattice gases. Princeton university Press, 1986.

[65] R.B. Israel. Some generic results in Mathematical Physics. Mark. Proc. Rel. Field. 10, no 3:517-523, 2004.

[66] E.T. Jaynes. E. T. Jaynes: papers on probability, statistics and statistical physics. Reprint of the 1983 original. Edited and introduced by R. D. Rosenkrantz. Pallas Paperbacks, 50. Kluwer Academic Publishers Group, Dordrecht, 1989.

[67] M. Kac, G. Uhlenbeck, P. Hemer. On the van der waals theory of vapor-liquid equilibrium. J. Math. Phys 4:216-228, 1963.

[68] T. Kennedy. Majority rule at low temperatures on the square and triangular lattices. J. Stat. Phys. 86, no. 5-6, 1089-1107, 1997.

[69] A.I. Khinchin. Mathematical foundations of information theory. New York : Dover, 1957.

[70] H.A. Kramers, G.H. Wannier. Statistics of the two-dimensional ferromagnet I, II. Phys. Rev. 2, no 60:252-262 and 263-276, 1941.

[71] S. Kobe. Ernst Ising, 1900-1998. Braz. J. Phys. 30, no.4: 649654,2000 .

[72] O. Kozlov. Gibbs description of a system of random variables. Problems Inform. Transmission. 10:258-265, 1974.

[73] C. Külske. (Non-) Gibbsianness and phase transition in random lattice spin models. Mark. Proc. Rel. Field. 5:357-383, 1999. 
[74] C. Külske. Weakly Gibbsian representation for joint measures of quenched lattice spin models. Prob. Th. Relat. Fields 119:1-30, 2001.

[75] C. Külske. Analogues of non-Gibbsianness in joint-measures of disordered mean-field models. J. Stat. Phys. 112, no 5/6:10791108, 2003.

[76] C. Külske. How non-Gibbsianness helps a metastable Morita minimizer to provide a stable free energy. Mark. Proc. Relat. Field. 10, no. 3: 547-564, 2004.

[77] C. Külske, A. Le Ny. Spin-flip dynamics of the Curie-Weiss model: Loss of Gibbsianness with possibly broken symmetry. Comm. Math. Phys. 271, vol 2:431-454, 2007.

[78] C. Külske, A. Le Ny, F. Redig. Relative entropy and variational properties of generalized Gibbsian measures. Ann. Proba. 32, no. 2:1691-1726, 2004.

[79] R. Kühn. Gibbs vs Non-Gibbs in the Equilibrium Ensemble approach to disordered systems. Mark. Proc. Relat. Field. 10, no. 3: 523-546, 2004.

[80] O.E. Lanford. Entropy and Equilibrium States in Classical Statistical Mechanics. In Statistical Mechanics and Mathematical Problems, Battelle Seattle 1971 Rencontres, Lectures Notes in Physics no 20, Springer-Verlag, Berlin etc., 1973.

[81] O.E. Lanford, D. Ruelle. Observables at infinity and states with short range correlations in statistical mechanics. Comm. Math. Phys. 13:194-215, 1969.

[82] A. Le Ny. Fractal failure of quasilocality for a majority rule transformation on a tree. Lett. Math. Phys. 54, no. 1:11-24, 2000 .

[83] A. Le Ny. Gibbsian Description of Mean-Field Models. In In and out equilibrium 2, Progress in Probability, vol. 60:463-480, Birkhäuser Verlag Basel, 2008.

[84] A. Le Ny, F. Redig. Short times conservation of Gibbsianness under local stochastic evolutions. J. Stat. Phys. 109, nos 5/6:1073-1090, 2002.

[85] A. Le Ny, F. Redig. Large deviation principle at fixed time in Glauber evolutions. Mark. Proc. Relat. Fields 10, no 1:65-74, 2004. 
[86] J. Lebowitz. Statistical mechanics of systems of unbounded spins. Comm. Math. Phys. 50:195-218, 1976.

[87] J. Lebowitz, O.E Penrose. On the exponential decay of correlations. Comm. Math. Phys. 39:165-184, 1974.

[88] J. Lebowitz, C. Maes. Entropy: a dialogue. Entropy: 269-276, Princeton Ser. Appl. Math., Princeton Univ. Press, Princeton, NJ, 2003.

[89] J. Lebowitz, R.H. Schonmann. Pseudo-free energies and Large deviations for Non Gibbsian FKG measures. Prob. Th. Relat. Field. 77:49-64, 1988

[90] R. Lefevere. Variational principle for some renormalized measures. J. Stat. Phys. 96, nos 1-2, 109-133, 1999.

[91] R. Lefevere. Weakly Gibbsian measures and quasilocality: a long range pair-interaction example. J. Stat. Phys. 95, no 3/4:785-789, 1999.

[92] J.T. Lewis, C.-E. Pfister. Thermodynamic probability theory: some aspects of large deviations. Uspekhi Mat. Nauk $\mathbf{5 0}$ no. 2(302):47-88; translation in Russian Math. Surveys 50, no. $2: 279-317,1995$.

[93] J.T. Lewis, C-E. Pfister, W.G. Sullivan. Entropy, concentration of probability and conditional limit theorems. Mark. Proc. Relat. Fields 1, no 3:319-386, 1995.

[94] T. M. Liggett. Interacting particle systems. Grundlehren der Mathematischen Wissenschaften [Fundamental Principles of Mathematical Sciences, 276. Springer-Verlag, New York, 1985.

[95] J. Lörinczi, C. Maes. Weakly Gibbsian measures for lattice spin systems. J. Stat. Phys. 89, no. 3-4:561-579, 1997.

[96] J. Lörinczi, C. Maes, K. Vande Velde. Transformations of Gibbs measures. Prob. Theo. Relat. Field. 112, no. 1, 121-147, 1998.

[97] C. Maes, F. Redig, A. Van Moffaert. Almost Gibbsian versus Weakly Gibbsian. Stoch. Proc. Appl. 79, no 1:1-15, 1999.

[98] C. Maes, F. Redig, A. Van Moffaert. The restriction of the Ising model to a Layer. J. Stat. Phys. 96, nos 1/2:69-107, 1999.

[99] C. Maes, K. Vande Velde. Relative energies for non-Gibbsian states. Comm. Math. Phys. 189, no 2:277-286, 1997. 
[100] E. Mahé. Utilisation des spécifications. PhD thesis, University of Rouen (France), November 30th 2007.

[101] P.-A. Meyer. Probabilities and potential. North Holland mathematics studies, Amsterdam: North-Holland, 1978.

[102] L. Onsager. Crystal statistics. I. A two-dimensional model with an order-disorder transition. Phys. Rev. 65 no 2:117-149, 1944.

[103] R. B. Peierls. On Ising's model of ferromagnetism. Proc. Cambridge Philos. Soc. 32:477-481, 1936.

[104] C.-E. Pfister. Thermodynamical aspects of classical lattice systems. In In and out of equilibrium. Probability with a physical flavor. Progress in Probability, Vladas Sidoravicius ed., Birkhaüser, pp 393-472, 2002.

[105] A. Petri, M. De Oliveira. Temperature of non-equilibrium lattice systems. Intern. J. Mod. Phys. C 17, no 12:1703-1715, 2006.

[106] S.A. Pirogov, Y. Sinai. Phase Diagram of Classical Lattice Systems. Theor. Math. Phys 25:1185-1192, 1975 and 26:39-49, 1976.

[107] C.Preston. Gibbs states on Countable sets. Cambridge tracts in Math., no 68, Cambridge University Press, London, New York, 1974.

[108] C.Preston. Random Fields. Lecture Notes in Mathematics 534, Springer-Verlag, 1976.

[109] B. Prum. Processus sur un réseau et mesures de Gibbs. Techniques stochastiques, Masson, Paris, 1986.

[110] D. Ruelle. Equilibrium states of infinite systems in statistical mechanics. Mathematical aspects of statistical mechanics (Proc. Sympos. Appl. Math., New York, 1971). SIAM-AMS Proceedings, Vol. V:47-53, Amer. Math. Soc., Providence, R. I., 1972.

[111] L. Russo. The infinite cluster method in the two-dimensional Ising model. Comm. Math. Phys. 67, no. 3, 251-266, 1979.

[112] R.H. Schonmann. Projection of Gibbs measures may be nonGibbsian. Comm. Math. Phys. 124:1-7, 1989.

[113] B. Simon. The statistical mechanics of lattice gases. Vol. I. Princeton Series in Physics. Princeton University Press, Princeton, NJ, 1993. 
[114] F. Spitzer. Phase transition in one-dimensional nearestneighbors systems, J. Funct. Anal. 20:240-255, 1975.

[115] J. Stoyanov. Counterexamples in probability. Wiley series in probability and mathematical statistics, Wiley, 1987.

[116] W.G. Sullivan. Finite range random fields and energy fields. J. Math. Anal. Appl. 44:710-724, 1973.

[117] W.G. Sullivan. Potentials for almost Markovian random fields, Comm. Math. Phys. 33:61-74, 1976.

[118] D. Williams. Probability with martingales. Cambridge mathematical textbooks, Cambridge University Press, 1991.

[119] K.G. Wilson. The renormalization group: Critical phenomena and the Kondo problem, Rev. Mod. Phys. 47:773-840, 1975.

[120] S. Xu. An ergodic process of zero divergence distance from the class of all stationary processes. J. Theo. Prob. 11, no 1:181$195,1988$.

[121] C.N. Yang. The spontaneous magnetization of a twodimensional Ising model. Phys. Rev. 85 no 2: 808-816, 1952.

\author{
Arnaud Le Ny \\ Université de Paris-Sud \\ Laboratoire de Mathématiques \\ Bâtiment 425 \\ 91405 Orsay Cedex, France \\ arnaud.leny@math.u-psud.fr \\ http://www.math.u-psud.fr/ leny
}

